\title{
Eleven years' data of grassland management in Germany
}

\author{
Juliane Vogt ${ }^{\ddagger}$, Valentin H. Klaus§,l, Steffen Both ${ }^{\ddagger}, \pi$, Cornelia Fürstenau\#, Sonja Gockel ${ }^{\mathbf{a}, ",}$, Martin M.

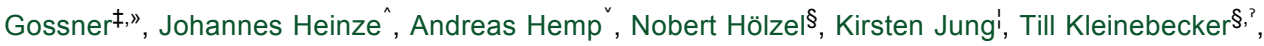 \\ Ralf Lauterbach', Katrin Lorenzen ${ }^{\ddagger}$, Andreas Ostrowski ${ }^{\#}$, Niclas Otto ${ }^{\ddagger}$, Daniel Prati ${ }^{\varsigma}$, Swen Renner ${ }^{\Phi}$, \\ Uta Schumacher ${ }^{\ell}$, Sebastian Seibold ${ }^{\ddagger}$, Nadja K. Simons ${ }^{\ddagger, \$}$, Iris Steitz', Miriam Teuscher ${ }^{\ell}$, Jan Thiele \\ P, Sandra Weithmann', Konstans Wells ${ }^{A}$, Kerstin Wiesner ${ }^{\ddagger}$, Manfred Ayassei, Nico Blüthgen ${ }^{-\beta}$, \\ Markus Fischer ${ }^{\varsigma}, \ell$, Wolfgang W. Weisser ${ }^{\ddagger}$ \\ ‡ Technische Universität München, School of Life Sciences Weihenstephan, Terrestrial Ecology Research Group, Freising, \\ Germany \\ $\S$ Westfälische Wilhelms-Universität, Institute of Landscape Ecology, Münster, Germany \\ | ETH Zürich, Institute of Agricultural Sciences, Zürich, Switzerland \\ ף Martin-Luther-Universität Halle-Wittenberg, Institut für Agrar- und Ernährungswissenschaften, Halle, Germany \\ \# Friedrich Schiller Universität Jena, Institute for Computer Science, Heinz Nixdorf Chair for Distributed Information Systems, Jena, \\ Germany \\ a Friedrich Schiller Universität Jena, Institute of Ecology, Jena, Germany \\ " Georg-August-Universität Göttingen, Silviculture and Forest Ecology of the Temperate Zones, Göttingen, Germany \\ "Swiss Federal Research Institute WSL, Forest Entomology, Birmensdorf, Switzerland \\ ^ Universität Potsdam, Biodiversity Research/Systematic Botany, Institute of Biochemistry and Biology, Potsdam, Germany \\ v University of Bayreuth, Department of Plant Systematics, Bayreuth, Germany \\ University of Ulm, Institute of Evolutionary Ecology and Conservation Genomics, Ulm, Germany \\ ? Justus-Liebig-Universität Gießen, Institute of Landscape Ecology and Resource Management, Gießen, Germany \\ ` University of Bern, Institute of Plant Science, Department of Biology, Bern, Switzerland \\ \ University of Natural Resources and Life Sciences BOKU, Institute of Zoology, Vienna, Austria \\ £ Senckenberg Gesellschaft für Naturforschung, Biodiversity and Climate Research Centre BiK-F, Frankfurt, Germany \\ \& Technische Universität Darmstadt, Ecological Networks, Darmstadt, Germany \\ P Johann Heinrich von Thünen Institute for Biodiversity, Braunschweig, Germany \\ A Swansea University, Department of Biosciences, Swansea, United Kingdom
}

Corresponding author: Juliane Vogt (juliane.vogt@tum.de)

Academic editor: Quentin Groom

Received: 21 May 2019 | Accepted: 19 Sep 2019 | Published: 27 Sep 2019

Citation: Vogt J, Klaus VH, Both S, Fürstenau C, Gockel S, Gossner MM, Heinze J, Hemp A, Hölzel N, Jung K, Kleinebecker T, Lauterbach R, Lorenzen K, Ostrowski A, Otto N, Prati D, Renner S, Schumacher U, Seibold S, Simons N, Steitz I, Teuscher M, Thiele J, Weithmann S, Wells K, Wiesner K, Ayasse M, Blüthgen N, Fischer M, Weisser WW (2019) Eleven years' data of grassland management in Germany. Biodiversity Data Journal 7: e36387. https://doi.org/10.3897/BDJ.7.e36387

(c) Vogt $\mathrm{J}$ et al. This is an open access article distributed under the terms of the Creative Commons Attribution License (CC BY 4.0), which permits unrestricted use, distribution, and reproduction in any medium, provided the original author and source are credited. 


\section{Abstract}

\section{Background}

The 150 grassland plots were located in three study regions in Germany, 50 in each region. The dataset describes the yearly grassland management for each grassland plot using 116 variables.

General information includes plot identifier, study region and survey year. Additionally, grassland plot characteristics describe the presence and starting year of drainage and whether arable farming had taken place 25 years before our assessment, i.e. between 1981 and 2006. In each year, the size of the management unit is given which, in some cases, changed slightly across years.

Mowing, grazing and fertilisation were systematically surveyed:

Mowing is characterised by mowing frequency (i.e. number of cuts per year), dates of cutting and different technical variables, such as type of machine used or usage of conditioner.

For grazing, the livestock species and age (e.g. cattle, horse, sheep), the number of animals, stocking density per hectare and total duration of grazing were recorded. As a derived variable, the mean grazing intensity was then calculated by multiplying the livestock units with the duration of grazing per hectare [LSU days/ha]. Different grazing periods during a year, partly involving different herds, were summed up to an annual grazing intensity for each grassland.

For fertilisation, information on the type and amount of different types of fertilisers was recorded separately for mineral and organic fertilisers, such as solid farmland manure, slurry and mash from a bioethanol factory. Our fertilisation measures neglect dung dropped by livestock during grazing. For each type of fertiliser, we calculated its total nitrogen content, derived from chemical analyses by the producer or agricultural guidelines (Table 3).

All three management types, mowing, fertilisation and grazing, were used to calculate a combined land use intensity index (LUI) which is frequently used to define a measure for the land use intensity. Here, fertilisation is expressed as total nitrogen per hectare [kg N/ ha], but does not consider potassium and phosphorus.

Information on additional management practices in grasslands was also recorded including levelling, to tear-up matted grass covers, rolling, to remove surface irregularities, seed addition, to close gaps in the sward. 


\section{New information}

Investigating the relationship between human land use and biodiversity is important to understand if and how humans affect it through the way they manage the land and to develop sustainable land use strategies. Quantifying land use (the ' $X$ ' in such graphs) can be difficult as humans manage land using a multitude of actions, all of which may affect biodiversity, yet most studies use rather simple measures of land use, for example, by creating land use categories such as conventional vs. organic agriculture. Here, we provide detailed data on grassland management to allow for detailed analyses and the development of land use theory. The raw data have already been used for $>100$ papers on the effect of management on biodiversity (e.g. Manning et al. 2015).

\section{Keywords}

Grassland management survey, fertilisation, grazing, mowing, livestock units, BiodiversityExploratories, questionnaire, farming practice, grassland maintenance, nitrogen, temporal variation, intensification of grassland use

\section{Introduction}

Grasslands can harbour high biodiversity and fulfil important ecosystem functions and services, such as food and habitat provision for livestock, protection of soil and water resources, carbon sequestration and aesthetic appeal (Carlier et al. 2009, Hönigová et al. 2012, Gossner et al. 2016, Simons et al. 2017). In addition to the conversion of grasslands to other land use forms, grasslands worldwide are also changed by land use intensification. Land use intensification of grasslands includes, for example, increased fertiliser input, application of pesticides, increased number of cuts in meadows or increased stocking densities in pastures (Humbert et al. 2009, Boch et al. 2016, Klaus et al. 2018. As a result of continued land use intensification, high value natural grasslands, i.e. extensively managed grasslands, have seen a decline throughout Europe (Veen et al. 2009).

Increasing management intensity in grasslands has been shown to decrease alpha, (i.e. local, diversity) and also beta diversity, i.e. intensification leads to homogenisation of communities across trophic groups including plant, invertebrates and birds (Humbert et al. 2009, Gossner et al. 2016, Manning et al. 2015, Renner et al. 2014, Socher et al. 2013). Intensification affects biodiversity directly and indirectly. For example, mowing itself and the use of conditioners, i.e. a farm implement that uses mechanical force to promote faster and more even drying of biomass, cause direct mortality of insects (Humbert et al. 2010a, Humbert et al. 2010b). Indirect effects include changes in plant community composition, for example, by increased fertilisation, that can then affect insect diversity.

Until now, little attention has been paid to long-term in-depth assessments of land use practices in grassland systems. The intensity and timing of mowing, grazing and fertilisation can differ within and between years on particular grasslands (Kleinebecker et 
al. 2018) and the effect of such variability on biodiversity changes is considerable (but see, e.g. Allan et al. 2014). Grassland management consists of various management components such as mowing, grazing or fertilisation that may jointly or singly affect biodiversity. Moreover, there are interactions between different management activities, for example, fertiliser application results in higher biomass production, which is often associated with more frequent mowing (Blüthgen et al. 2012, Busch et al. 2018, Humbert et al. 2009Busch et al. 2018). To understand more mechanistically how land use intensification in grasslands affects biodiversity, detailed information on grassland management is needed, ideally for a large number of grasslands over several years.

Within the framework of the Biodiversity-Exploratories programme (www.biodiversityexploratories.de), we have thoroughly monitored land use of 150 grassland plots for 11 years to investigate temporal variation in land management within three study regions in Germany (Fig. 1). These plots represented gradients of land use intensity typical for our study regions and were managed by mowing, grazing and fertilisation (Fischer et al. 2010). Detailed information on grassland management of all 150 grassland plots was obtained annually from farmers using a standardised questionnaire (Table 1). Here, we present the data of the corresponding management questionnaire that form the basis of most analyses of effects of land use intensification on biodiversity and ecosystem functioning in grasslands within the Biodiversity-Exploratories. With this dataset, we provide knowledge on how land use intensity in temperate grasslands varies across spatial and temporal scales. The components reported here also form the basis on an integrated land use intensity index used in the programme to study its integral effects on biodiversity in grasslands (Blüthgen et al. 2012).

Table 1.

Overview of all variables of the data set: BE_landuse_grassland_2006-2016.csv received from the management questionnaire.

\begin{tabular}{|c|c|c|c|c|}
\hline Variable & $\begin{array}{l}\text { Type of } \\
\text { data }\end{array}$ & Units & $\begin{array}{l}\text { Range of } \\
\text { numeric } \\
\text { variables } \\
\text { (min-max) }\end{array}$ & Description (English) \\
\hline ID & Text & - & - & $\begin{array}{l}\text { Unique identifier composed of the } \\
\text { columns PlotID and Year }\end{array}$ \\
\hline Study region & Text & & - & $\begin{array}{l}\text { ALB = Schwäbische Alb } \\
\mathrm{HAI}=\text { Hainich } \\
\mathrm{SCH}=\text { Schorfheide }\end{array}$ \\
\hline Year & Integer & yyyy & - & Year of management \\
\hline Date & Date & dd.mm.yyyy & - & Date of interview \\
\hline
\end{tabular}




\begin{tabular}{|c|c|c|c|c|}
\hline Variable & $\begin{array}{l}\text { Type of } \\
\text { data }\end{array}$ & Units & $\begin{array}{l}\text { Range of } \\
\text { numeric } \\
\text { variables } \\
\text { (min-max) }\end{array}$ & Description (English) \\
\hline PlotlD & Text & - & - & $\begin{array}{l}\text { Experimental Plot IDs formatted as }(\mathrm{A}|\mathrm{H}| \\
\text { S)EG with consecutive numbering. } \\
\text { Abbreviations are: } \\
A=\text { Schwäbische Alb, } \mathrm{H}=\text { Hainich, } \mathrm{S}= \\
\text { Schorfheide } \\
\mathrm{E}=\text { Experimental Plot } \\
G=\text { Grassland, e.g. AEG01 }\end{array}$ \\
\hline Drainage & Text & - & - & $\begin{array}{l}\text { Measure of drainage and the description } \\
\text { of the method (free text) }\end{array}$ \\
\hline StartDrainage & Integer & yyyy & - & $\begin{array}{l}\text { Starting year of grassland drainage, if } \\
\text { applicable }\end{array}$ \\
\hline WaterLogging & Boolean & yes/no & - & $\begin{array}{l}\text { Activities on water logging, e.g. for water } \\
\text { regulation of fen soils }\end{array}$ \\
\hline Agriculture1981 & Boolean & yes/no & - & $\begin{array}{l}\text { Use of grassland between } 1981 \text { to } 2006 \text {, } \\
\text { i.e. (temporal) conversion of grassland } \\
\text { into arable land }\end{array}$ \\
\hline SizeManagementUnit_ha & Numeric & ha & $0.49-187.1$ & $\begin{array}{l}\text { Size of the management unit in the } \\
\text { survey year, often larger than the } 50 \mathrm{x} \\
50 \mathrm{~m} \text { study plot itself }\end{array}$ \\
\hline \multicolumn{5}{|l|}{ Grazing } \\
\hline StartGrazing & Text & Month & - & $\begin{array}{l}\text { Starting month of the first grazing period } \\
\text { in the survey year }\end{array}$ \\
\hline EndGrazing & Text & Month & - & $\begin{array}{l}\text { End of the last grazing period in the } \\
\text { survey year }\end{array}$ \\
\hline Livestock1 & Text & & - & Type of animal in first grazing period \\
\hline StartGrazingPeriod1 & Text & month & - & $\begin{array}{l}\text { Starting month of first grazing period for } \\
\text { livestock } 1\end{array}$ \\
\hline EndGrazingPeriod1 & Text & month & - & $\begin{array}{l}\text { Ending month of first grazing period for } \\
\text { livestock } 1\end{array}$ \\
\hline $\begin{array}{l}\text { Livestock, Start/End } \\
\text { GrazingPeriod 2-4 ... }\end{array}$ & & & - & $\begin{array}{l}\text { Identical information for grazing periods } \\
2-4 \text {, if applicable. }\end{array}$ \\
\hline Cattle6months 1 & Integer & & $0-95$ & $\begin{array}{l}\text { For Grazing period } 1 \text { : Number of cattle } \\
\text { with an age up to } 6 \text { months (cattle up to } \\
6 \text { months }=0.3 \text { LS }^{*} \text { per day) }\end{array}$ \\
\hline Cattle6-24months 1 & Integer & & $0-200$ & $\begin{array}{l}\text { For Grazing period 1: Number of cattle } \\
\text { with an age between } 6 \text { months and } 2 \\
\text { years }\left(=0.6 \text { LS }^{*}\right)\end{array}$ \\
\hline CattlePlus2years1 & Integer & & $0-300$ & $\begin{array}{l}\text { For Grazing period } 1 \text { : Number of cattle } \\
\text { older than } 2 \text { years }\left(=1 \mathrm{LS}^{\star}\right)\end{array}$ \\
\hline SheepGoat1year1 & Integer & & $0-1000$ & $\begin{array}{l}\text { For Grazing period } 1 \text { : Number of sheep } \\
\text { or goats with an age up to } 1 \text { year }(=0.05 \\
\left.\text { LS }^{*}\right)\end{array}$ \\
\hline
\end{tabular}




\begin{tabular}{|c|c|c|c|c|}
\hline Variable & $\begin{array}{l}\text { Type of } \\
\text { data }\end{array}$ & Units & $\begin{array}{l}\text { Range of } \\
\text { numeric } \\
\text { variables } \\
\text { (min-max) }\end{array}$ & Description (English) \\
\hline SheepGoatPlus1year1 & Integer & & $0-1500$ & $\begin{array}{l}\text { For Grazing period 1: Number of sheep } \\
\text { or goats older than } 1 \text { year }\left(=0.1 \mathrm{LS}^{*}\right)\end{array}$ \\
\hline Pony1 & Integer & & $0-400$ & $\begin{array}{l}\text { For Grazing period 1: Number of ponies } \\
\text { and small horses }\left(=0.7 \mathrm{LS}^{\star}\right)\end{array}$ \\
\hline Horse3years1 & Integer & & $0-4$ & $\begin{array}{l}\text { For Grazing period 1: Number of horses } \\
\text { up to } 3 \text { years }\left(=0.7 \mathrm{LS}^{\star}\right)\end{array}$ \\
\hline HorsePlus3years1 & Integer & & $0-46$ & $\begin{array}{l}\text { For Grazing period 1: Number of horses } \\
\text { older than } 3 \text { years }\left(=1.1 \mathrm{LS}^{*}\right)\end{array}$ \\
\hline NbLivestock1 & Integer & & $0-2500$ & $\begin{array}{l}\text { For Grazing period 1: Total number of } \\
\text { livestock }\end{array}$ \\
\hline LivestockUnits1 & Numeric & $\begin{array}{l}\text { Number of livestock } \\
x \text { conversion factor }\end{array}$ & $0-1814$ & $\begin{array}{l}\text { For Grazing period 1: Total sum of the } \\
\text { livestock units }\end{array}$ \\
\hline DayGrazing1 & Integer & days & $0-365$ & $\begin{array}{l}\text { For Grazing period 1: duration of } \\
\text { grazing (in days) }\end{array}$ \\
\hline GrazingArea1 & Numeric & ha & $0-148.5$ & $\begin{array}{l}\text { For Grazing period 1: size of area where } \\
\text { livestock grazed }\end{array}$ \\
\hline \multicolumn{5}{|c|}{ Numeric variables for grazing 2-4...For Grazing periods 2-4 see description of grazing period 1} \\
\hline Cattle6months2 & & & $0-73$ & \\
\hline Cattle6-24months2 & & & $0-103$ & \\
\hline CattlePlus2years2 & & & $0-120$ & \\
\hline SheepGoat1year2 & & & $0-600$ & \\
\hline SheepGoatPlus1year2 & & & $0-1200$ & \\
\hline Pony2 & & & 0 & \\
\hline Horse3years2 & & & 0 & \\
\hline HorsePlus3years2 & & & $0-18$ & \\
\hline NbLivestock2 & & & $0-1200$ & \\
\hline LivestockUnits2 & & & $0-144$ & \\
\hline DayGrazing2 & & & $0-165$ & \\
\hline GrazingArea2 & & & $0-148.5$ & \\
\hline Cattle6months3 & & & $0-72$ & \\
\hline Cattle6-24months3 & & & $0-103$ & \\
\hline CattlePlus2years3 & & & $0-120$ & \\
\hline SheepGoat1year3 & & & $0-820$ & \\
\hline SheepGoatPlus1year3 & & & $0-1300$ & \\
\hline Pony3 & & & 0 & \\
\hline Horse3years3 & & & 0 & \\
\hline HorsePlus3years3 & & & $0-25$ & \\
\hline
\end{tabular}




\begin{tabular}{|c|c|c|c|c|}
\hline Variable & $\begin{array}{l}\text { Type of } \\
\text { data }\end{array}$ & Units & $\begin{array}{l}\text { Range of } \\
\text { numeric } \\
\text { variables } \\
\text { (min-max) }\end{array}$ & Description (English) \\
\hline NbLivestock3 & & & $0-1340$ & \\
\hline LivestockUnits3 & & & $0-145.5$ & \\
\hline DayGrazing3 & & & $0-127$ & \\
\hline GrazingArea3 & & & $0-196.69$ & \\
\hline Cattle6months4 & & & $0-72$ & \\
\hline Cattle6-24months4 & & & $0-81$ & \\
\hline CattlePlus2years 4 & & & $0-84$ & \\
\hline SheepGoat1year4 & & & $0-600$ & \\
\hline SheepGoatPlus1year4 & & & $0-900$ & \\
\hline Pony4 & & & 0 & \\
\hline Horse3years 4 & & & 0 & \\
\hline HorsePlus3years 4 & & & $0-16$ & \\
\hline NbLivestock4 & & & $0-900$ & \\
\hline LivestockUnits4 & & & $0-103.6$ & \\
\hline DayGrazing4 & & & $0-76$ & \\
\hline GrazingArea4 & & & $0-148.5$ & \\
\hline TotalGrazing_LSUdha & Numeric & \#Livestock*days /ha & $0-1644.17$ & $\begin{array}{l}\text { Total sum of the grazing intensity for all } \\
\text { grazing periods }\end{array}$ \\
\hline SupplementaryFeeding & Boolean & yes/no & - & Additional fodder supply for the livestock \\
\hline DescFeeding & Text & & - & $\begin{array}{l}\text { Type and amount of supplementary } \\
\text { fodder }\end{array}$ \\
\hline \multicolumn{5}{|l|}{ Mowing } \\
\hline Mowing & Integer & 1/year & $0-4$ & Number of cuts per year \\
\hline DateMowing1 & Date & dd.mm.yyyy & - & Date of the first cut \\
\hline DateMowing2-4... & Date & dd.mm.yyyy & - & $\begin{array}{l}\text { Dates of the second to fourth cut, if } \\
\text { applicable }\end{array}$ \\
\hline Mowing Machine & Text & & - & $\begin{array}{l}\text { Type of machine which was used for } \\
\text { mowing, e.g. rotarymower, doubleknife, } \\
\text { mulcher }\end{array}$ \\
\hline CutWidth_m & Numeric & $\mathrm{m}$ & $0-12$ & Cutting width of the mowing machine \\
\hline CutHeight_cm & Integer & $\mathrm{cm}$ & $0-15$ & $\begin{array}{l}\text { Cutting height above soil level of the } \\
\text { mowing machine }\end{array}$ \\
\hline DriveSpeed_kmh & Integer & $\mathrm{km} / \mathrm{h}$, (mean) & $0-20$ & $\begin{array}{l}\text { Speed of the mowing machine, normally } \\
\text { mean speed value is given }\end{array}$ \\
\hline MowingConditioner & Boolean & yes/no & - & $\begin{array}{l}\text { Presence of conditioner, i.e. did the } \\
\text { mowing machine have a conditioner to } \\
\text { improve drying of the clippings }\end{array}$ \\
\hline
\end{tabular}




\begin{tabular}{|c|c|c|c|c|}
\hline Variable & $\begin{array}{l}\text { Type of } \\
\text { data }\end{array}$ & Units & $\begin{array}{l}\text { Range of } \\
\text { numeric } \\
\text { variables } \\
\text { (min-max) }\end{array}$ & Description (English) \\
\hline \multicolumn{5}{|l|}{ Fertilisation } \\
\hline Fertilisation & Boolean & yes/no & - & $\begin{array}{l}\text { Addition of fertiliser (not including dung } \\
\text { depositions by livestock during grazing a } \\
\text { parcel) }\end{array}$ \\
\hline NbFertilisation & Integer & & $0-7$ & Number of fertiliser applications per year \\
\hline DateFertilisation1 & Date & dd.mm.yyyy & - & Date of first fertiliser application \\
\hline DateFertilisation2-7... & Date & dd.mm.yyyy & - & Date of 2nd to 7th fertiliser applications \\
\hline Manure_tha & Numeric & t/ha & $0-40$ & Total amount of applied solid manure \\
\hline Slurry_m3ha & Numeric & $\mathrm{m}^{3} / \mathrm{ha}$ & $0-80$ & $\begin{array}{l}\text { Total amount of applied pig or cow } \\
\text { slurry and biogas residues, respectively. }\end{array}$ \\
\hline DescFert & Text & & - & Description of applied organic fertiliser \\
\hline orgNitrogen_kgNha & Numeric & $\mathrm{kg} / \mathrm{ha}$ & $0-371$ & Amount of organic nitrogen applied \\
\hline minNitrogen_kgNha & Numeric & $\mathrm{kg} / \mathrm{ha}$ & $0-170$ & $\begin{array}{l}\text { Amount of nitrogen applied, of mineral } \\
\text { origin or the organic fertiliser mash from } \\
\text { a bioethanol factory (see in DescFert) }\end{array}$ \\
\hline totalNitrogen_kgNha & Numeric & $\mathrm{Kg} / \mathrm{ha}$ & $0-433$ & $\begin{array}{l}\text { Sum of applied mineral and organic } \\
\text { nitrogen [kg N/ha] }\end{array}$ \\
\hline minPhosphorus_kgPha & Numeric & $\mathrm{kg} / \mathrm{ha}$ & $0-350$ & $\begin{array}{l}\text { Amount of phosphorus applied [ } \mathrm{kg} \mathrm{P}_{2} \mathrm{O}_{5} \\
\text { /ha], of mineral origin or mash from a } \\
\text { bioethanol factory (not given for other } \\
\text { organic fertilisers) }\end{array}$ \\
\hline minPotassium_kgKha & Numeric & $\mathrm{kg} / \mathrm{ha}$ & $0-100$ & $\begin{array}{l}\text { Amount of potassium applied }\left[\mathrm{kg} \mathrm{K}_{2} \mathrm{O} /\right. \\
\text { ha], of mineral origin or mash from a } \\
\text { bioethanol factory (not given for other } \\
\text { organic fertilisers) }\end{array}$ \\
\hline Sulphur_kgSha & Numeric & $\mathrm{kg} / \mathrm{ha}$ & $0-25$ & $\begin{array}{l}\text { Total amount of applied Sulphur [kg S/ } \\
\text { ha] }\end{array}$ \\
\hline \multicolumn{5}{|l|}{ Maintenance } \\
\hline Maintenance & Boolean & yes/no & - & Presence of maintenance measures \\
\hline Levelling & Text & & $0-4$ & $\begin{array}{l}\text { Maintenance to break up matted grass } \\
\text { covers }\end{array}$ \\
\hline DateLevelling & Date & dd.mm.yyyy & - & Maintenance: date of levelling \\
\hline Rolling & Text & & $0-2$ & $\begin{array}{l}\text { Maintenance: rolling to level } \\
\text { unevenness }\end{array}$ \\
\hline DateRolling & Date & dd.mm.yyyy & - & Maintenance: date of rolling \\
\hline Mulching & Text & & $0-4$ & $\begin{array}{l}\text { Partial mulching on some spots, e.g. } \\
\text { rank patches. The material remains on } \\
\text { site after mowing. We consider this not } \\
\text { as a mowing event as only a small part } \\
\text { of the area is treated. }\end{array}$ \\
\hline
\end{tabular}




\begin{tabular}{|c|c|c|c|c|}
\hline Variable & $\begin{array}{l}\text { Type of } \\
\text { data }\end{array}$ & Units & $\begin{array}{l}\text { Range of } \\
\text { numeric } \\
\text { variables } \\
\text { (min-max) }\end{array}$ & Description (English) \\
\hline DateMulching & Date & dd.mm.yyyy & - & Date of partial mulching \\
\hline ShrubClearance & Text & - & $0-1$ & $\begin{array}{l}\text { Clearance to avoid shrub } \\
\text { encroachment. We consider this not as } \\
\text { a mowing event as only individual } \\
\text { shrubs are targeted. }\end{array}$ \\
\hline DateScrubCl & Date & dd.mm.yyyy & - & Date of shrub clearance \\
\hline PlantProtectionAgent & Boolean & yes/no & - & $\begin{array}{l}\text { Pesticide use: pesticides and } \\
\text { herbicides. As pesticides in grasslands } \\
\text { are very rare and only used for spot } \\
\text { treatment, we do not have further } \\
\text { information on this treatment. }\end{array}$ \\
\hline Seeds & Boolean & yes/no & - & Seed addition \\
\hline DescSeeds & Text & - & - & Description of usage of the sowing \\
\hline
\end{tabular}

${ }^{*}$ LS - Livestock

Table 2.

Livestock units derived from the type and age of livestock (Chamber of Agriculture NordrheinWestfalen 2018).

\begin{tabular}{|l|l|l|}
\hline Grazing species & Age & Livestock units (LSU) \\
\hline Cattle & $<6$ months & 0.3 \\
\hline Cattle & 6 months-2 years & 0.6 \\
\hline Cattle & $>2$ years & 1 \\
\hline Sheep and goats & $<1$ year & 0.05 \\
\hline Sheep and goats & $>1$ year & 0.1 \\
\hline Ponies and small horses & - & 0.7 \\
\hline Horses & $<3$ years & 0.7 \\
\hline Horses & $>3$ years & 1.1 \\
\hline
\end{tabular}

\section{General description}

Purpose: The present dataset summaries management information collected from 2006 to 2016 for 150 grassland plots in three different regions of Germany. Data are based on annual interviews with the respective farmers, land owners or tenants involved in land management activity, using a standardised questionnaire. 


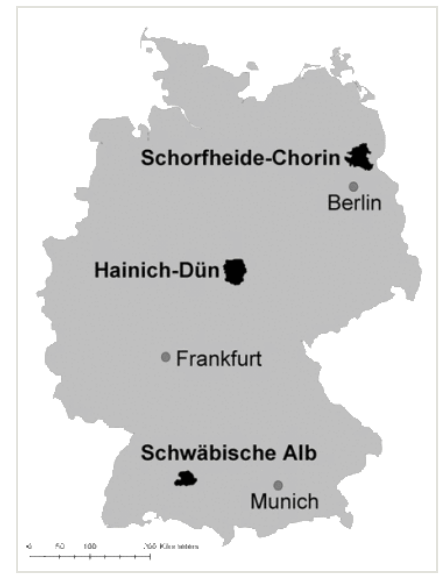

Figure 1. doi

The three model regions of the Biodiversity Exploratories project in Germany.

\section{Project description}

Title: The Biodiversity Exploratories - functional biodiversity research

Personnel: Members of the steering committee of the BE: Markus Fischer, Wolfgang Weisser, Manfred Ayasse, Christian Ammer, Nico Blüthgen, Ellen Kandeler, Birgitta KönigRies, Marion Schrumpf.

Within the infrastructure programme of the BE, local management teams in each region ensure the maintenance of survey plots and communication between scientists and local stakeholders. Furthermore, the grassland expert (technician) and the local manager (scientist) of each team are responsible for obtaining the information from the land user by carrying out the annual questionnaire, as well as including additional information by their own observations of the grasslands.

Study area description: The biodiversity studies are carried out in 150 grassland plots managed in different intensities.

The grassland sites are distributed in three different regions within Germany including i) the Biosphere Reserve Schorfheide-Chorin ii) the Hainich-Dün Area and iii) the BiosphereArea Schwäbische Alb.

The Schorfheide Chorin Exploratory site is situated in the North-East of Germany with an extent of approx. $1300 \mathrm{~km}^{2}$. The geology is characterised by young glacial landscape of altitudes between 3-140 m a.s.l. with different soil types such as brown earth, lessivé, pararendzina, podzols and bog soils, resulting in diverse vegetation. The annual mean temperature is $8-8.5^{\circ} \mathrm{C}$ and the annual mean precipitation $500-600 \mathrm{~mm}$. 
The Hainich-Dün Exploratory site (approx. $1300 \mathrm{~km}^{2}$ ) in Central Germany consists of silty, loamy and clayey soil textures of the calcareous bedrock in altitudes between 285- $550 \mathrm{~m}$ a.s.I. The annual mean temperature is $6.5-8^{\circ} \mathrm{C}$ and the annual mean precipitation $500-800$ $\mathrm{mm}$.

The Exploratory Schwäbische Alb site (approx. $422 \mathrm{~km}^{2}$ ) in South West Germany consists of calcareous bedrock with karst phenomena in altitudes between 460-860 $\mathrm{m}$ a.s.l. with annual mean temperature of $6-7^{\circ} \mathrm{C}$ and mean precipitation of $700-1000 \mathrm{~mm}$.

Design description: For an advanced biodiversity research, three large-scale and longterm research sites were established in Germany serving as open research platforms for biodiversity and ecosystem research groups. The BE sustained the scientific infrastructure to develop the intellectual framework needed to address critical questions about changes in biodiversity and to evaluate the impacts of those changes for ecosystem processes.

The objectives of the BE are to understand i) the relationship between biodiversity of different taxa and levels, ii) the role of land use and management for biodiversity, iii) the role of biodiversity for ecosystem processes.

Funding: The Biodiversity Exploratories are a German Science Foundation funded research project (DFG Priority Programme 1374).

\section{Sampling methods}

Study extent: We monitored 150 grassland plots across three regions in Germany for 11 years since 2006 .

Sampling description: Interviews with the land users took place retrospectively for the previous year on all permanently established 150 grassland sites since 2006 , based on a standardised questionnaire, which was identical for all three exploratory regions.

We did not collect any organisms. During the interviews, the land users provided us with information according to their grassland management.

Linear mixed-effect models with logarithmically transformed response variables were calculated to detect temporal trends as well as differences between the exploratory regions (procedure Imer, implemented in R).

Land use intensity in the grasslands of our study regions ranged from low-intensive management, for example, meadows with only one cut per year and no fertilisation, to intensive management with four cuts per year and occasionally up to $400 \mathrm{~kg} \mathrm{~N}$ added per year and hectare. Very intensively-used grasslands which, in Central Europe, are characterised by up to seven cuts per year and regular fertilisation of about $400 \mathrm{~kg} / \mathrm{ha} / \mathrm{yr}$ nitrogen did not occur in our study regions. 
Mean mowing frequency (number of cuts per year) across all 50 plots was between 0.6 and 1.5 and highest in the Alb, lower in Hainich and lowest in Schorfheide. Mowing frequency slightly increased in the Alb and Hainich, but decreased over the years in the Schorfheide. Within plots that were mown, mowing intensity was between 1.3 and 2 cuts per year and was highest in Alb, significantly higher than in Schorfheide (Fig. 2a). Mowing frequency within mown plots decreased over time in Schorfheide (Fig. 2a).

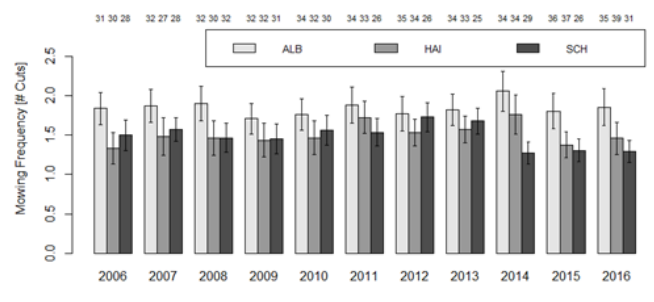

a)

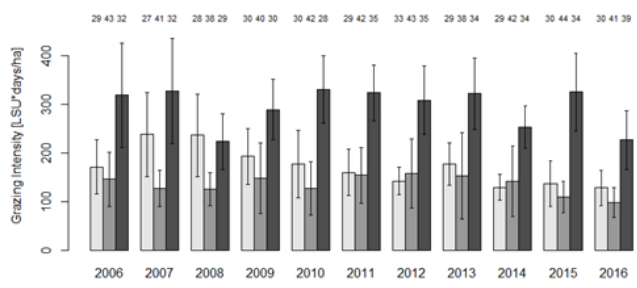

b)

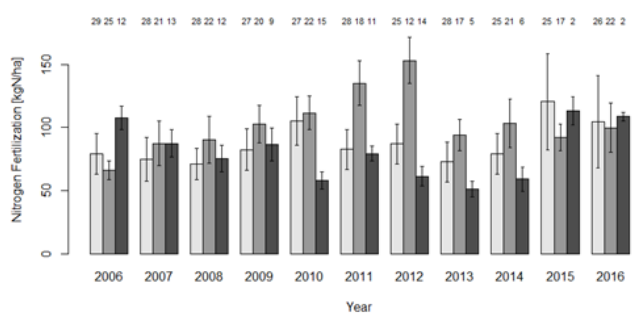

c)

Figure 2. doi

Annual means and standard error for a) mowing frequency in mown plots, i.e. the number of cuts per year, b) grazing intensity in grazed plots, in livestock unit days per hectare and year, calculated by multiplying the number of livestock by a conversion factor (see Table 3 ) and the number of grazing days and dividing the product by the size of the management unit and c) nitrogen fertilisation in fertilised plots, calculated as total nitrogen input in $\mathrm{kg}$ per hectare and year, in the three study regions of the Biodiversity Exploratories (light grey: Schwäbische Alb (ALB), grey: Hainich-Dün (HAI) and dark grey: Schorfheide-Chorin $(\mathrm{SCH})$ ). Only the subset of plots (out of 50 in each region), where the respective management was applied, are included in the figure panels (numbers above the bars). 
Table 3.

Nitrogen input conversion factor of manure and slurry.

\begin{tabular}{|c|c|c|}
\hline $\begin{array}{l}\text { Type of } \\
\text { manure (t/ } \\
\text { ha) }\end{array}$ & $\begin{array}{l}\text { Conversion Factor } \\
\text { for total Nitrogen } \\
{[\mathrm{kg} / \mathrm{t}]}\end{array}$ & Literature and Notes \\
\hline Cattle & 5.6 & \multirow{3}{*}{$\begin{array}{l}\text { LWK (Chamber of Agriculture) Nordrhein-Westfalen (2014), own measurements } \\
\text { analysed by LUFA Nord-West (Agricultural Investigation and Research Institute - } \\
\text { accredited laboratory of the Chamber of Agriculture in Niedersachen) (2017) }\end{array}$} \\
\hline Horse & 4.9 & \\
\hline Sheep & 8.13 & \\
\hline $\begin{array}{l}\text { Type of } \\
\text { slurry }\left(\mathrm{m}^{3} /\right. \\
\text { ha) }\end{array}$ & $\begin{array}{l}\text { Total Nitrogen }[\mathrm{kg} / \mathrm{m} \\
\text { 3] }\end{array}$ & \\
\hline Cattle & $3.85(3.2-4.5)$ & \multirow[t]{3}{*}{ Mean values of slurry ranges were used. (LWK Nordrhein-Westfalen(2014)) } \\
\hline Pig & $5.4(4.3-6.5)$ & \\
\hline Mixed & $4.45(4.0-4.9)$ & \\
\hline $\begin{array}{l}\text { Biogas / } \\
\text { Digestate }\end{array}$ & 4.4 & LWK Baden-Württemberg (2012) \\
\hline
\end{tabular}

Grasslands were grazed by different types of livestock, most commonly cattle and sheep, but also horses and goats. Based on this information, the mean grazing intensity was then calculated by multiplying the livestock units ([LSU], (Table 2) with the duration of grazing per hectare [LSU days/ha]. Grazing intensity across all 50 plots in a region was on average between 120 and 200 livestock unit days per hectare in the Schorfheide, significantly higher than in the Alb $(z=3.177, p<0.01)$ where yearly means were mostly below 100 . Mean grazing intensity in Hainich was intermediate with yearly means below 150 (data not shown). In the Schorfheide, grazing intensity across the 50 plots increased slightly over time $(z=6.091, p<0.0001$, data not shown). In grazed plots, the annual grazing intensity per hectare ranged from 5 to 1644 livestock units $x$ days. Mean grazing intensity in grazed plots was higher in the Schorfheide than in the other two regions, but due to high variability, differences between regions were not significant ( $p>0.05$, Fig. $2 b$ ). Within the grazed plots, grazing intensity in Schorfheide decreased over time $(z=-3.270, p<0.01$, Fig. $2 b$ ), although the number of plots that were grazed were higher in the second half of the time series (Fig. 2b).

Fertilisation intensity across the 50 plots was highest in Hainich, with means mainly higher than $20 \mathrm{~kg} \mathrm{~N}^{*} \mathrm{ha}^{-1 *} \mathrm{yr}^{-1}$, significantly higher than in the Schorfheide $(z=2.343, \mathrm{p}<0.05)$, where there was a significant decrease in fertilisation with time $(z=-5.017, p<0.001)$ and where yearly means dropped from $20 \mathrm{~kg} \mathrm{~N} \mathrm{ha}^{-1} \mathrm{yr}^{-1}$ to close to zero after 2013, which is largely due to a decrease in the number of fertilised plots to just two (Fig. 2c). Fertilisation in the Alb was intermediate (data not shown). Within fertilised plots, fertilisation ranged between 15 and $433 \mathrm{~kg} \mathrm{~N}^{-1} \mathrm{yr}^{-1}$ and there were no differences between regions or changes over time ( $p>0.05$ in each case, Fig. $2 c)$. 
To summarise, there were significant differences between the regions in main grassland use, meadows in the Alb and pasture in Schorfheide and also in mean land use intensity of meadows, pastures or mown pastures. Changes over time were largely due to changes in the number of plots that were grazed, mown or fertilised, rather than to changes in mowing, grazing and fertilisation intensity within plots. In the Schorfheide, there was an overall decrease in land use intensity, due to increasing regulations in the biosphere reserve Schorfheide Chorin. In the Hainich, the number of fertilised plots decreased from 25 plots in 2006 to 12 plots in 2012 and then increased again to 22 in 2016 (Fig. 2c).

The management of grassland is decisively influenced by subsidies, such as agrienvironmental measures (AEM) (Table 4). These AEMs are different within the federal states of Germany, having names such as MEKA or FAKT in Baden-Wuerttemberg and KULAP in Thuringia and Brandenburg, including single measures of different management aspects. The agri-environmental subsidy programmes aim to support environmental friendly and extensive production practices to protect natural resources and to preserve cultural landscapes. These can also be counted as disadvantage compensations and are co-financed by the EU, Germany and the respective federal state. Measures of these progammes determine guidelines regarding organic farming, the timing and type of mowing and grazing or restrictions, according to plant protection agents or fertiliser use (Table 4). Therefore, farmers do not make completely independent decisions by managing their grasslands but follow the regulations of the agri-environmental measures to receive subsidies for their land.Table 5 lists the agri-environmental measures applied for the single study plots for each year. The description of the coding of the agri-environmental measures is found as a legend in Table 6.

Table 4.

The requirements of single agri-environmental measures (MEKA/FAKT for Baden-Wuerttemberg and KULAP for Thuringia and Brandenburg) are characterised by the subprogramme designation listed for every region (ALB- Swabian Alb, HAl- Hainich, SCH- Schorfheide). The abbreviations (R)LSU mean (roughage consuming) livestock units having a livestock-dependent conversion from LSU to RLSU: 1 LSU equals the RLSU for sheep or goat (0.7), horse (0.5), cattle (1).

\begin{tabular}{|c|c|c|c|}
\hline Agri-environmental measures & $\begin{array}{l}\text { ALB (MEKA, } \\
\text { FAKT) }\end{array}$ & HAI (KULAP) & SCH (KULAP) \\
\hline \multicolumn{4}{|l|}{ Requirements } \\
\hline Difficult management due to slope of $\geq 25 \%$ & N-B3 & & \\
\hline $\begin{array}{l}\text { Adapted, extensive management of biotope ( } \$ 32 \\
\text { nature conservation) }\end{array}$ & N-G1.1, B4 & & \\
\hline FFH: lowlands- and mountain-meadows & B5 & & \\
\hline $\begin{array}{l}\text { Conservation of meadow orchards (eligible up to } \\
\max 100 \text { trees/ha) }\end{array}$ & C1 & & \\
\hline $\begin{array}{l}\text { Low-nutrient and dry habitats (biotopes maintenance } \\
\text { by grazing) }\end{array}$ & & N21, G21 & \\
\hline
\end{tabular}




\begin{tabular}{|c|c|c|c|}
\hline Agri-environmental measures & $\begin{array}{l}\text { ALB (MEKA, } \\
\text { FAKT) }\end{array}$ & HAI (KULAP) & SCH (KULAP) \\
\hline $\begin{array}{l}\text { Low-nutrient and dry habitats (biotopes maintenance } \\
\text { by mowing) }\end{array}$ & & N31 & \\
\hline Wet meadows & & N23 & \\
\hline Eligible landscape (e.g. Natura 2000) & & & $\begin{array}{l}413 \mathrm{~A}, 423 \mathrm{~B}, 613 \mathrm{~A}, \\
663\end{array}$ \\
\hline Sheep farming and difficult terrain & & N25 & \\
\hline $\begin{array}{l}\text { Difficult conditions (regarding terrain, specific } \\
\text { management) }\end{array}$ & & G31, G33, G53 & \\
\hline Location of valuable genetic plants & & L4 & \\
\hline Compensatory allowance of disadvantaged sites & & & 33 \\
\hline \multicolumn{4}{|l|}{ Organic farming } \\
\hline Farm is managed according to EU eco-regulation & N-D2, D2 & & \\
\hline $\begin{array}{l}\text { Introduction or retention of ecological management } \\
\text { of the farm }\end{array}$ & & L1, Ö2 & $773,673,882$ \\
\hline $\begin{array}{l}\text { Retention of ecological management - compensatory } \\
\text { allowance }\end{array}$ & & & $623 \mathrm{~A}, \mathrm{~B}, \mathrm{C}, \mathrm{D}$ \\
\hline \multicolumn{4}{|l|}{ In general } \\
\hline Main fodder site & B1.2 & & \\
\hline Min $5 \%$ of eligible site managed after 15 June & N-B1, N-B3 & & \\
\hline Promoting of endangered livestock breeds & C3 & & \\
\hline No reduction of permanent grassland of the farm & & N25 & \\
\hline $\begin{array}{l}\text { Management plan according to nature conservation } \\
\text { authority }\end{array}$ & & $\begin{array}{l}\text { N231, N31, G21, } \\
\text { G31, G33, G53 }\end{array}$ & 663 \\
\hline $\begin{array}{l}\text { Fodder sites are managed at least once per year by } \\
\text { grazing or mowing }\end{array}$ & & & $\begin{array}{l}\text { 413A, 423B, 613A, } \\
663,673\end{array}$ \\
\hline Management after 1 July & & & $812 \mathrm{C}$ \\
\hline \multicolumn{4}{|l|}{ Grazing } \\
\hline Livestock min $0.3 \mathrm{LSU} / \mathrm{ha}$ on agriculture area & & Ö2 & \\
\hline Livestock max $2 \mathrm{LSU} / \mathrm{ha}$ on agriculture area & $\mathrm{N}-\mathrm{B} 1, \mathrm{~N}-\mathrm{B} 3$ & & 773,673 \\
\hline Livestock min 0.5 RLSU/ha fodder area & & L4 & \\
\hline Livestock min $0.3 \mathrm{RLSU} / \mathrm{ha}$ fodder area & N-B2, B1.1, B1.2 & & $\begin{array}{l}311 \mathrm{~A}, 311 \mathrm{C}, 773 \\
673,661,411\end{array}$ \\
\hline Livestock max 1.4 RLSU/ ha & & & $\begin{array}{l}311 \mathrm{~A}, 311 \mathrm{C}, 773 \\
673,661,411\end{array}$ \\
\hline Livestock max 1.4 LSU/ ha & N-B2, B1.1 & & $311 \mathrm{~A}, 311 \mathrm{C}$ \\
\hline At least one grazing per year & & N25 & \\
\hline
\end{tabular}




\begin{tabular}{|c|c|c|c|}
\hline Agri-environmental measures & $\begin{array}{l}\text { ALB (MEKA, } \\
\text { FAKT) }\end{array}$ & HAI (KULAP) & SCH (KULAP) \\
\hline $\begin{array}{l}\text { At least one grazing per year. First grazing period by } \\
\text { cattle/horses or sheep/goats }\end{array}$ & & G21 & \\
\hline $\begin{array}{l}\text { At least one grazing per year. First grazing period by } \\
\text { sheep/goats }\end{array}$ & & G33, G53 & \\
\hline $\begin{array}{l}\text { At least one management per year (grazing or } \\
\text { mowing and harvesting of the yield) before } 15 \\
\text { October }\end{array}$ & & & 411,661 \\
\hline Maintenance measures after grazing & N-B1, N-B2, N-B3 & & \\
\hline Grazing by cattle/horses with $0.3-1$ LSU/ha & & N211 & \\
\hline $\begin{array}{l}\text { Grazing by cattle/horses with permanent grazing or } \\
\text { at least from } 2 \text { May to } 15 \text { October }\end{array}$ & & G31 & \\
\hline Grazing by sheep or goats with a min $0.5 \mathrm{LSU} / \mathrm{ha}$ & & N213, N25, G33, G53 & \\
\hline Grazing 0.3-1 LSU/ha & & N231 & \\
\hline Max 1.5 LSU/ha*d until 1 July & & N231 & \\
\hline $\begin{array}{l}\text { First management of the year: at least } 80 \% \text { of area } \\
\text { by grazing (up to } 20 \% \text { by mowing) }\end{array}$ & & N231 & \\
\hline $\begin{array}{l}\text { First management mowing: grazing possible at least } \\
7 \text { weeks after the first cut }\end{array}$ & & N31 & \\
\hline No supplementary feeding & & N233 & \\
\hline $\begin{array}{l}\text { No supplementary feeding between } 1 \text { May and } 15 \\
\text { October }\end{array}$ & & G21, G31, G33, G53 & \\
\hline \multicolumn{4}{|l|}{ Mowing } \\
\hline First management mowing and harvesting the yield & & N31 & \\
\hline Up to 2 cuts with a time lag of at least 7 weeks & & N31 & \\
\hline $\begin{array}{l}\text { First mowing not before } 15 \text { August on min } 5 \% \text { of the } \\
\text { area }\end{array}$ & & N31 & \\
\hline No mowing before 16 June & & & $313 \mathrm{~A}$ \\
\hline No mowing before 1 July & & & $313 B$ \\
\hline Post grazing mowing not before 1 July & & $\begin{array}{l}\text { N231, G31, G33, } \\
\text { G53 }\end{array}$ & \\
\hline Cut height $10 \mathrm{~cm}$ & & & 313B, 763 \\
\hline \multicolumn{4}{|l|}{ Indicator plant species } \\
\hline $\begin{array}{l}\text { Abundance of at least } 4 \text { indicator plant species out of } \\
28 \text { specific forbs }\end{array}$ & N-B4 & L4 & \\
\hline $\begin{array}{l}\text { Abundance of at least } 6 \text { indicator plant species out of } \\
30 \text { specific plants }\end{array}$ & B3.2 & G11 & \\
\hline
\end{tabular}




\begin{tabular}{|c|c|c|c|}
\hline Agri-environmental measures & $\begin{array}{l}\text { ALB (MEKA, } \\
\text { FAKT) }\end{array}$ & HAI (KULAP) & SCH (KULAP) \\
\hline $\begin{array}{l}\text { Abundance of at least } 7 \text { indicator plant species out of } \\
\text { specific plants }\end{array}$ & B5 & & \\
\hline \multicolumn{4}{|l|}{ Fertilisation } \\
\hline No mineral nitrogen fertilisation & B1.1 & & \\
\hline No mineral or organic nitrogen fertilisation & B1.2 & & \\
\hline No slurry fertilisation & & & $311 \mathrm{C}$ \\
\hline No fertilisation & & & $811 \mathrm{~A}$ \\
\hline $\begin{array}{l}\text { No chemical-synthetic fertiliser or plant protection } \\
\text { agent within the farm }\end{array}$ & D1 & & $\begin{array}{l}\text { 311A, 311C, 661, } \\
411\end{array}$ \\
\hline $\begin{array}{l}\text { No chemical-synthetic fertiliser or plant protection } \\
\text { agent on eligible areas }\end{array}$ & & N231, N25; N31 & \\
\hline No fertiliser or plant protection agent & & G21, G31, G33 & \\
\hline \multicolumn{4}{|l|}{ Documentation } \\
\hline Slurry records (amount, date) for eligible areas & N-B1, N-B3 & & \\
\hline $\begin{array}{l}\text { Fertilisation and management records for eligible } \\
\text { areas }\end{array}$ & N-B4 & & \\
\hline Fertilisation and mowing records for eligible areas & B3.2 & & \\
\hline $\begin{array}{l}\text { Fertilisation and plant protection agent records for all } \\
\text { grasslands of the farm }\end{array}$ & B1.2 & & \\
\hline $\begin{array}{l}\text { Records via Thuringian grassland card for eligible } \\
\text { areas }\end{array}$ & & $\begin{array}{l}\text { N231, N25, N31, } \\
\text { G21, G31, G33, G53 }\end{array}$ & \\
\hline \multicolumn{4}{|l|}{ Restrictions /measures not taken } \\
\hline No ploughing, only seed addition & B1.1, B1.2, B3.2 & & \\
\hline \multicolumn{4}{|l|}{ No ploughing on eligible areas } \\
\hline No ploughing on farm & $\begin{array}{l}\text { N-B1, N-B2, N-B3, } \\
\text { N-B4, N-D2 }\end{array}$ & & $\begin{array}{l}413 \mathrm{~A}, 423 \mathrm{~B}, 411, \\
661\end{array}$ \\
\hline No irrigation or melioration & N-B2, B1.1, B1.2 & G21, G31, G33, G53 & \\
\hline No extensive usage of plant protection agents & $\begin{array}{l}\text { N-B1, N-B2, N-B3, } \\
\text { N-B4, B1.1, B1.2 }\end{array}$ & & \\
\hline $\begin{array}{l}\text { No maintenance measures, mowing or seed addition } \\
\text { between } 1 \text { April and } 30 \text { June }\end{array}$ & & G21, G31, G33, G53 & \\
\hline No upturning or limbering tillage & & G21, G31, G33, G53 & \\
\hline
\end{tabular}

\section{Table 5.}

Study plots with the geographical coordinates and the coding of the agri-environmental measures. The description of coding is found in the legend of Table 6 . 


\begin{tabular}{|c|c|c|c|c|c|c|c|c|c|c|c|c|c|c|}
\hline EP_Plot_ID & Explo & Latitude & Longitude & 2006 & 2007 & 2008 & 2009 & 2010 & 2011 & 2012 & 2013 & 2014 & 2015 & 2016 \\
\hline AEG1 & ALB & 48.4 & 9.34 & k 0 n 0 & $\mathrm{k} 0 \mathrm{n} 0$ & $\mathrm{k} 0 \mathrm{n} 0$ & $\mathrm{k} 0 \mathrm{nO}$ & $\mathrm{k} 0 \mathrm{nO}$ & $\mathrm{k} 0 \mathrm{nO}$ & $\mathrm{k} 0 \mathrm{n} 0$ & $\mathrm{k} 0 \mathrm{nO}$ & $\mathrm{k} 0 \mathrm{n} 0$ & $\mathrm{k} 0 \mathrm{n} 0$ & $\mathrm{k} 0 \mathrm{nO}$ \\
\hline AEG10 & ALB & 48.38 & 9.21 & $\begin{array}{l}\text { o } 0 \mathrm{n} \\
\text { A5 }\end{array}$ & $\begin{array}{l}\text { o } 0 \mathrm{n} \\
\text { A5 }\end{array}$ & $\begin{array}{l}\text { o } 0 \mathrm{n} \\
\text { A5 }\end{array}$ & $\begin{array}{l}\text { o } 0 \mathrm{n} \\
\text { A5 }\end{array}$ & $\begin{array}{l}\text { o } 0 \mathrm{n} \\
\text { A5 }\end{array}$ & $\begin{array}{l}\text { o } 0 n \\
\text { A5 }\end{array}$ & $\begin{array}{l}\text { o } 0 \mathrm{n} \\
\text { A5 }\end{array}$ & $\begin{array}{l}\text { o } 0 \mathrm{n} \\
\text { A5 }\end{array}$ & $\begin{array}{l}\text { o } 0 n \\
\text { A5 }\end{array}$ & $\mathrm{k} 0 \mathrm{n} 0$ & $\mathrm{k} 0 \mathrm{nO}$ \\
\hline AEG11 & ALB & 48.49 & 9.35 & k 0 n 0 & $\mathrm{k} 0 \mathrm{n} 0$ & $\mathrm{k} 0 \mathrm{n} 0$ & $\mathrm{k} 0 \mathrm{n} 0$ & $\mathrm{k} 0 \mathrm{nO}$ & $\mathrm{k} 0 \mathrm{n} 0$ & $\mathrm{k} 0 \mathrm{n} 0$ & $\mathrm{k} 0 \mathrm{n} 0$ & $\mathrm{k} 0 \mathrm{nO}$ & $\mathrm{k} 0 \mathrm{n} 0$ & $\mathrm{k} 0 \mathrm{n} 0$ \\
\hline AEG12 & ALB & 48.39 & 9.35 & k 0 n 0 & $\mathrm{k} 0 \mathrm{n} 0$ & $\mathrm{k} 0 \mathrm{n} 0$ & $\mathrm{k} 0 \mathrm{nO}$ & $\mathrm{k} 0 \mathrm{nO}$ & $\mathrm{k} 0 \mathrm{n} 0$ & $\mathrm{k} 0 \mathrm{n} 0$ & $\mathrm{k} 0 \mathrm{nO}$ & $\mathrm{k} 0 \mathrm{nO}$ & $\mathrm{k} 0 \mathrm{n} 0$ & $\mathrm{k} 0 \mathrm{nO}$ \\
\hline AEG13 & ALB & 48.39 & 9.36 & k 0 n 0 & $\mathrm{k} 0 \mathrm{n} 0$ & $\mathrm{k} 0 \mathrm{n} 0$ & $\mathrm{k} 0 \mathrm{nO}$ & $\mathrm{k} 0 \mathrm{nO}$ & $\mathrm{k} 0 \mathrm{nO}$ & $\mathrm{k} 0 \mathrm{nO}$ & $\mathrm{k} 0 \mathrm{nO}$ & $\mathrm{k} 0 \mathrm{nO}$ & $\mathrm{k} 0 \mathrm{n} 0$ & $\mathrm{k} 0 \mathrm{n} 0$ \\
\hline AEG14 & ALB & 48.38 & 9.52 & k 0 n 0 & $\mathrm{k} 0 \mathrm{n} 0$ & $\mathrm{k} 0 \mathrm{nO}$ & $\mathrm{k} 0 \mathrm{nO}$ & $\mathrm{k} 0 \mathrm{nO}$ & $\mathrm{k} 0 \mathrm{nO}$ & $\mathrm{k} 0 \mathrm{n} 0$ & $\mathrm{k} 0 \mathrm{nO}$ & $\mathrm{k} 0 \mathrm{nO}$ & $\mathrm{k} 0 \mathrm{n} 0$ & $\mathrm{k} 0 \mathrm{nO}$ \\
\hline AEG15 & ALB & 48.49 & 9.45 & k 0 n 0 & $\mathrm{k} 0 \mathrm{n} 0$ & $\mathrm{k} 0 \mathrm{n} 0$ & $\mathrm{k} 0 \mathrm{n} 0$ & $\mathrm{k} 0 \mathrm{nO}$ & $\mathrm{k} 0 \mathrm{n} 0$ & $\mathrm{k} 0 \mathrm{n} 0$ & $\mathrm{k} 0 \mathrm{n} 0$ & $\mathrm{k} 0 \mathrm{nO}$ & $\mathrm{k} 0 \mathrm{n} 0$ & $\begin{array}{l}\mathrm{k} 0 \mathrm{y} \\
\mathrm{A} 12\end{array}$ \\
\hline AEG16 & ALB & 48.4 & 9.46 & $\begin{array}{l}\text { ocy } \\
\text { A4/A5 }\end{array}$ & $\begin{array}{l}\text { o c y } \\
\text { A4/A5 }\end{array}$ & $\begin{array}{l}\text { ocy } \\
\text { A4/A5 }\end{array}$ & $\begin{array}{l}\text { ocy } \\
\text { A4/A5 }\end{array}$ & $\begin{array}{l}\text { o c y } \\
\text { A4/A5 }\end{array}$ & $\begin{array}{l}\text { o c y } \\
\text { A4/A5 }\end{array}$ & $\begin{array}{l}\text { o cy } \\
\text { A4/A5 }\end{array}$ & $\begin{array}{l}\text { o c y } \\
\text { A4/A5 }\end{array}$ & $\begin{array}{l}\text { o c y } \\
\text { A4/A5 }\end{array}$ & $\begin{array}{l}\text { o c y } \\
\text { A14/11/ } \\
\text { A12 }\end{array}$ & $\begin{array}{l}\text { oc y } \\
\text { A14/ } \\
\text { A16/ } \\
\text { A12 }\end{array}$ \\
\hline AEG17 & ALB & 48.4 & 9.52 & o a y 0 & o a y 0 & o a y 0 & o a y 0 & o a y 0 & o a y 0 & o a y 0 & o a y 0 & o a y 0 & oc 00 & oc 00 \\
\hline AEG18 & ALB & 48.38 & 9.52 & k 0 n 0 & $\mathrm{k} 0 \mathrm{n} 0$ & $\mathrm{k} 0 \mathrm{n} 0$ & $\mathrm{k} 0 \mathrm{n} 0$ & $\mathrm{k} 0 \mathrm{nO}$ & $\mathrm{k} 0 \mathrm{nO}$ & $\mathrm{k} 0 \mathrm{n} 0$ & $\mathrm{k} 0 \mathrm{nO}$ & $\mathrm{k} 0 \mathrm{nO}$ & $\mathrm{k} 0 \mathrm{n} 0$ & $\mathrm{k} 0 \mathrm{n} 0$ \\
\hline AEG19 & ALB & 48.4 & 9.45 & k 0 n 0 & $\mathrm{k} 0 \mathrm{n} 0$ & $\mathrm{k} 0 \mathrm{n} 0$ & $\mathrm{k} 0 \mathrm{nO}$ & $\mathrm{k} 0 \mathrm{nO}$ & $\mathrm{k} 0 \mathrm{n} 0$ & $\mathrm{k} 0 \mathrm{nO}$ & $\mathrm{k} 0 \mathrm{nO}$ & $\mathrm{k} 0 \mathrm{nO}$ & $\mathrm{k} 0 \mathrm{n} 0$ & $\mathrm{k} 0 \mathrm{nO}$ \\
\hline AEG2 & ALB & 48.38 & 9.47 & k 0 n 0 & $\mathrm{k} 0 \mathrm{n} 0$ & $\mathrm{k} 0 \mathrm{nO}$ & $\mathrm{kOnO}$ & $\mathrm{k} 0 \mathrm{nO}$ & $\mathrm{k} 0 \mathrm{nO}$ & $\mathrm{k} 0 \mathrm{n} 0$ & $\mathrm{k} 0 \mathrm{nO}$ & $\mathrm{k} 0 \mathrm{nO}$ & $\mathrm{k} 0 \mathrm{n} 0$ & $\mathrm{k} 0 \mathrm{nO}$ \\
\hline AEG20 & ALB & 48.49 & 9.36 & k 0 n 0 & $\mathrm{k} 0 \mathrm{n} 0$ & $\mathrm{k} 0 \mathrm{n} 0$ & $\mathrm{k} 0 \mathrm{nO}$ & $\mathrm{k} 0 \mathrm{n} 0$ & $\mathrm{k} 0 \mathrm{n} 0$ & $\mathrm{k} 0 \mathrm{n} 0$ & $\mathrm{k} 0 \mathrm{n} 0$ & $\mathrm{k} 0 \mathrm{nO}$ & $\mathrm{k} 0 \mathrm{n} 0$ & $\mathrm{k} 0 \mathrm{nO}$ \\
\hline AEG21 & ALB & 48.44 & 9.36 & $\begin{array}{l}\text { o } 0 y \\
\text { A5 }\end{array}$ & $\begin{array}{l}\text { o } 0 y \\
\text { A5 }\end{array}$ & $\begin{array}{l}\text { o } 0 y \\
\text { A5 }\end{array}$ & $\begin{array}{l}\text { o } 0 y \\
\text { A5 }\end{array}$ & $\begin{array}{l}\text { o } 0 y \\
\text { A5 }\end{array}$ & $\begin{array}{l}\text { o } 0 y \\
\text { A5 }\end{array}$ & $\begin{array}{l}\text { o } 0 y \\
\text { A5 }\end{array}$ & $\begin{array}{l}\text { o } 0 y \\
\text { A5 }\end{array}$ & 0000 & $\mathrm{k} 0 \mathrm{n} 0$ & $\mathrm{k} 0 \mathrm{n} 0$ \\
\hline AEG22 & ALB & 48.4 & 9.51 & $\begin{array}{l}\text { k } 0 y \\
\text { A1 }\end{array}$ & $\begin{array}{l}\mathrm{k} 0 \mathrm{y} \\
\mathrm{A} 1\end{array}$ & $\begin{array}{l}\mathrm{k} 0 \mathrm{y} \\
\mathrm{A} 1\end{array}$ & $\begin{array}{l}\mathrm{k} 0 \mathrm{y} \\
\mathrm{A} 1\end{array}$ & $\begin{array}{l}\mathrm{k} 0 \mathrm{y} \\
\mathrm{A} 1\end{array}$ & $\begin{array}{l}\mathrm{k} 0 \mathrm{y} \\
\mathrm{A} 1\end{array}$ & $\begin{array}{l}\mathrm{k} 0 \mathrm{y} \\
\mathrm{A} 1\end{array}$ & $\begin{array}{l}\text { k } 0 y \\
\text { A1 }\end{array}$ & $\begin{array}{l}\mathrm{k} 0 \mathrm{y} \\
\mathrm{A} 1\end{array}$ & $\begin{array}{l}\text { k } 0 \text { y A7/ } \\
\text { A10 }\end{array}$ & $\begin{array}{l}\mathrm{k} 0 \mathrm{y} \\
\text { A7/A10 }\end{array}$ \\
\hline AEG23 & ALB & 48.42 & 9.51 & k 0 y 0 & k 0 y 0 & $\mathrm{k} 0$ y 0 & $\mathrm{k} 0$ y 0 & k 0 y 0 & $\mathrm{k} 0$ y 0 & $\mathrm{k} 0$ y 0 & k 0 y 0 & $\mathrm{k} 0$ y 0 & o с у A12 & $\begin{array}{l}\text { ocy } \\
\text { A12 }\end{array}$ \\
\hline AEG24 & ALB & 48.4 & 9.49 & k 0 y 0 & k 0 y 0 & $\mathrm{k} 0$ y 0 & k 0 y 0 & $\mathrm{k} 0$ y 0 & $\mathrm{k} 0$ y 0 & $\mathrm{k} 0$ y 0 & k 0 y 0 & k 0 y 0 & k 0 y A15 & $\begin{array}{l}\text { k } 0 y \\
\text { A15 }\end{array}$ \\
\hline AEG25 & ALB & 48.4 & 9.26 & k 0 y 0 & k 0 y 0 & $\mathrm{k} 0$ y 0 & $\mathrm{k} 0$ y 0 & $\mathrm{k} 0$ y 0 & $\mathrm{k} 0$ y 0 & $\mathrm{k} 0$ y 0 & $\mathrm{k} 0$ y 0 & $\mathrm{k} 0$ y 0 & k 0 y A19 & $\begin{array}{l}\mathrm{k} 0 \mathrm{y} \\
\mathrm{A} 19\end{array}$ \\
\hline AEG26 & ALB & 48.4 & 9.4 & k 0 y 0 & k 0 y 0 & $\mathrm{k} 0$ y 0 & $\mathrm{k} 0$ y 0 & $\mathrm{k} 0$ y 0 & $\mathrm{k} 0$ y 0 & $\mathrm{k} 0$ y 0 & k 0 y 0 & $\mathrm{k} 0$ y 0 & $\begin{array}{l}\text { k } 0 \text { y A15/ } \\
\text { A19 }\end{array}$ & $\begin{array}{l}\text { k } 0 \text { y } \\
\text { A15/ } \\
\text { A19 }\end{array}$ \\
\hline
\end{tabular}




\begin{tabular}{|c|c|c|c|c|c|c|c|c|c|c|c|c|c|c|}
\hline EP_Plot_ID & Explo & Latitude & Longitude & 2006 & 2007 & 2008 & 2009 & 2010 & 2011 & 2012 & 2013 & 2014 & 2015 & 2016 \\
\hline AEG27 & ALB & 48.42 & 9.48 & k 0 y 0 & k 0 y 0 & k 0 y 0 & k 0 y 0 & k 0 y 0 & $\mathrm{k} 0$ y 0 & k 0 y 0 & k 0 y 0 & $\mathrm{k} 0$ y 0 & $\begin{array}{l}\text { k } 0 \text { y A15/ } \\
\text { A17/A20/ } \\
\text { A19 }\end{array}$ & $\begin{array}{l}\text { k } 0 \text { y } \\
\text { A15/ } \\
\text { A17/ } \\
\text { A20/ } \\
\text { A19 }\end{array}$ \\
\hline AEG28 & ALB & 48.46 & 9.49 & k 0 y 0 & k 0 y 0 & $\mathrm{k} 0$ y 0 & k 0 y 0 & k 0 y 0 & $\mathrm{k} 0$ y 0 & k 0 y 0 & k 0 y 0 & k 0 y 0 & k 0 y A15 & $\begin{array}{l}\text { k } 0 y \\
\text { A15 }\end{array}$ \\
\hline AEG29 & ALB & 48.42 & 9.36 & k 0 y 0 & k 0 y 0 & $\mathrm{k} 0$ y 0 & $\mathrm{k} 0$ y 0 & k 0 y 0 & $\mathrm{k} 0$ y 0 & k 0 y 0 & k 0 y 0 & $\mathrm{k} 0$ y 0 & $\begin{array}{l}\text { k } 0 \text { y } A 9 / \\
\text { A11 }\end{array}$ & $\begin{array}{l}\mathrm{k} 0 \mathrm{y} \\
\mathrm{A} 9 / \mathrm{A} 11\end{array}$ \\
\hline AEG3 & ALB & 48.41 & 9.53 & $\begin{array}{l}\text { o a y } \\
\text { A5 }\end{array}$ & o a y 0 & o a y 0 & o a y 0 & o a y 0 & o a y 0 & o a y 0 & o a y 0 & oc y 0 & о с у A12 & $\begin{array}{l}\text { o cy } \\
\text { A12 }\end{array}$ \\
\hline AEG30 & ALB & 48.46 & 9.46 & k 0 y 0 & k 0 y 0 & $\mathrm{k} 0$ y 0 & k 0 y 0 & k 0 y 0 & $\mathrm{k} 0$ y 0 & k 0 y 0 & k 0 y 0 & $\mathrm{k} 0$ y 0 & k 0 y A15 & $\begin{array}{l}\text { k } 0 y \\
\text { A15 }\end{array}$ \\
\hline AEG31 & ALB & 48.46 & 9.46 & k 0 y 0 & k 0 y 0 & $\mathrm{k} 0$ y 0 & k 0 y 0 & k 0 y 0 & $\mathrm{k} 0$ y 0 & k 0 y 0 & k 0 y 0 & $\mathrm{k} 0$ y 0 & k 0 y A15 & $\begin{array}{l}\text { k } 0 y \\
\text { A15 }\end{array}$ \\
\hline AEG32 & ALB & 48.47 & 9.49 & k 0 y 0 & k 0 y 0 & $\mathrm{k} 0$ y 0 & k 0 y 0 & k 0 y 0 & $\mathrm{k} 0$ y 0 & k 0 y 0 & k 0 y 0 & k 0 y 0 & k 0 y A15 & $\begin{array}{l}\text { k } 0 y \\
\text { A15 }\end{array}$ \\
\hline AEG33 & ALB & 48.45 & 9.49 & k 0 y 0 & k 0 y 0 & $\mathrm{k} 0$ y 0 & k 0 y 0 & k 0 y 0 & $\mathrm{k} 0$ y 0 & k 0 y 0 & k 0 y 0 & $\mathrm{k} 0$ y 0 & $\begin{array}{l}\text { k } 0 \text { y } A 15 / \\
\text { A11 }\end{array}$ & $\begin{array}{l}\text { k } 0 y \\
\text { A15/ } \\
\text { A11 }\end{array}$ \\
\hline AEG34 & ALB & 48.46 & 9.5 & k 0 y 0 & k 0 y 0 & $\mathrm{k} 0$ y 0 & k 0 y 0 & k 0 y 0 & $\mathrm{k} 0$ y 0 & k 0 y 0 & k 0 y 0 & k 0 y 0 & k 0 y A15 & $\begin{array}{l}\mathrm{k} 0 \mathrm{y} \\
\mathrm{A} 15\end{array}$ \\
\hline AEG35 & ALB & 48.48 & 9.29 & $\mathrm{k} 0 \mathrm{n} 0$ & $\mathrm{k} 0 \mathrm{n} 0$ & $\mathrm{k} 0 \mathrm{nO}$ & $\mathrm{k} 0 \mathrm{n} 0$ & $\mathrm{k} 0 \mathrm{n} 0$ & $\mathrm{k} 0 \mathrm{n} 0$ & k 0 n 0 & $\mathrm{k} 0 \mathrm{n} 0$ & k 0 n 0 & $\mathrm{k} 0 \mathrm{n} 0$ & $\mathrm{k} 0 \mathrm{n} 0$ \\
\hline AEG36 & ALB & 48.48 & 9.3 & $\mathrm{k} 0 \mathrm{n} 0$ & $\mathrm{k} 0 \mathrm{n} 0$ & $\mathrm{k} 0 \mathrm{nO}$ & $\mathrm{k} 0 \mathrm{n} 0$ & $\mathrm{k} 0 \mathrm{n} 0$ & $\mathrm{k} 0 \mathrm{n} 0$ & $\mathrm{k} 0 \mathrm{n} 0$ & $\mathrm{k} 0 \mathrm{n} 0$ & $\mathrm{k} 0 \mathrm{n} 0$ & $\mathrm{k} 0 \mathrm{n} 0$ & $\mathrm{k} 0 \mathrm{n} 0$ \\
\hline AEG37 & ALB & 48.4 & 9.41 & $\mathrm{k} 0 \mathrm{n} 0$ & $\mathrm{k} 0 \mathrm{n} 0$ & $\mathrm{k} 0 \mathrm{n} 0$ & $\mathrm{k} 0 \mathrm{n} 0$ & $\mathrm{k} 0 \mathrm{n} 0$ & $\mathrm{k} 0 \mathrm{n} 0$ & k 0 n 0 & k 0 n 0 & k 0 n 0 & $\mathrm{k} 0 \mathrm{n} 0$ & k 0 n 0 \\
\hline AEG38 & ALB & 48.44 & 9.43 & k 0 y 0 & k 0 y 0 & $\mathrm{k} 0$ y 0 & k 0 y 0 & k 0 y 0 & $\mathrm{k} 0$ y 0 & k 0 y 0 & k 0 y 0 & k 0 y 0 & $\mathrm{k} 0 \mathrm{n} 0$ & k 0 n 0 \\
\hline AEG39 & ALB & 48.39 & 9.43 & $\mathrm{k} 0 \mathrm{n} 0$ & $\mathrm{k} 0 \mathrm{n} 0$ & $\mathrm{k} 0 \mathrm{nO}$ & $\mathrm{k} 0 \mathrm{n} 0$ & $\mathrm{k} 0 \mathrm{n} 0$ & $\mathrm{k} 0 \mathrm{nO}$ & k 0 n 0 & k 0 n 0 & k 0 n 0 & $\mathrm{k} 0 \mathrm{nO}$ & k 0 n 0 \\
\hline AEG4 & ALB & 48.38 & 9.42 & $\mathrm{k} 0 \mathrm{n} 0$ & $\mathrm{k} 0 \mathrm{n} 0$ & $\mathrm{k} 0 \mathrm{nO}$ & $\mathrm{k} 0 \mathrm{n} 0$ & $\mathrm{k} 0 \mathrm{n} 0$ & $\mathrm{k} 0 \mathrm{nO}$ & k 0 n 0 & k 0 n 0 & k 0 n 0 & $\mathrm{k} 0 \mathrm{nO}$ & k 0 n 0 \\
\hline AEG40 & ALB & 48.41 & 9.57 & $\begin{array}{l}\text { k } 0 \text { y } \\
\text { A1/ } \\
\text { A19 }\end{array}$ & $\begin{array}{l}\text { k } 0 y \\
\text { A1/ } \\
\text { A19 }\end{array}$ & $\begin{array}{l}\text { k } 0 y \\
\text { A1/ } \\
\text { A19 }\end{array}$ & $\begin{array}{l}\text { k } 0 y \\
\text { A1/ } \\
\text { A19 }\end{array}$ & $\begin{array}{l}\text { k } 0 \text { y } \\
\text { A1/ } \\
\text { A19 }\end{array}$ & $\begin{array}{l}\text { k0 y } \\
\text { A1/A19 }\end{array}$ & $\begin{array}{l}\text { k } 0 y \\
\text { A1/A19 }\end{array}$ & $\begin{array}{l}\text { k } 0 y \\
\text { A1/A19 }\end{array}$ & $\begin{array}{l}\text { k } 0 \text { y } \\
\text { A1/A19 }\end{array}$ & $\mathrm{k} 0 \mathrm{n} 0$ & k 0 n 0 \\
\hline AEG41 & ALB & 48.37 & 9.4 & $\begin{array}{l}\mathrm{k} 0 \mathrm{y} \\
\mathrm{A} 2\end{array}$ & $\begin{array}{l}\mathrm{k} 0 \mathrm{y} \\
\mathrm{A} 2\end{array}$ & $\begin{array}{l}\mathrm{k} 0 \mathrm{y} \\
\mathrm{A} 2\end{array}$ & $\begin{array}{l}\mathrm{k} 0 \mathrm{y} \\
\mathrm{A} 2\end{array}$ & $\begin{array}{l}\mathrm{k} 0 \mathrm{y} \\
\mathrm{A} 2\end{array}$ & $\begin{array}{l}\mathrm{k} 0 \mathrm{y} \\
\mathrm{A} 2\end{array}$ & $\begin{array}{l}\mathrm{k} 0 \mathrm{y} \\
\mathrm{A} 2\end{array}$ & $\begin{array}{l}\mathrm{k} 0 \mathrm{y} \\
\mathrm{A} 2\end{array}$ & $\begin{array}{l}\mathrm{k} 0 \mathrm{y} \\
\mathrm{A} 2\end{array}$ & $\mathrm{k} 0 \mathrm{nO}$ & k 0 n 0 \\
\hline AEG42 & ALB & 48.4 & 9.38 & k 0 y 0 & k 0 y 0 & $\mathrm{k} 0$ y 0 & k 0 y 0 & k 0 y 0 & $\mathrm{k} 0$ y 0 & k 0 y 0 & k 0 y 0 & $\mathrm{k} 0$ y 0 & $\mathrm{k} 0 \mathrm{n} 0$ & $\mathrm{k} 0 \mathrm{n} 0$ \\
\hline AEG43 & ALB & 48.41 & 9.54 & k 0 y 0 & k 0 y 0 & $\mathrm{k} 0$ y 0 & k 0 y 0 & k 0 y 0 & $\mathrm{k} 0$ y 0 & k 0 y 0 & k 0 y 0 & k 0 n 0 & $\mathrm{k} 0$ y $\mathrm{A} 8$ & $\mathrm{k} 0$ y $\mathrm{A} 8$ \\
\hline
\end{tabular}




\begin{tabular}{|c|c|c|c|c|c|c|c|c|c|c|c|c|c|c|}
\hline EP_Plot_ID & Explo & Latitude & Longitude & 2006 & 2007 & 2008 & 2009 & 2010 & 2011 & 2012 & 2013 & 2014 & 2015 & 2016 \\
\hline AEG44 & ALB & 48.38 & 9.43 & k 0 y 0 & $\mathrm{k} 0$ y 0 & $\mathrm{k} 0$ y 0 & $\mathrm{k} 0$ y 0 & $\mathrm{k} 0$ y 0 & $\mathrm{k} 0$ y 0 & $\mathrm{k} 0$ y 0 & $\mathrm{k} 0$ y 0 & $\mathrm{k} 0$ y 0 & $\mathrm{k} 0 \mathrm{n} 0$ & $\mathrm{k} 0 \mathrm{nO}$ \\
\hline AEG45 & ALB & 48.4 & 9.46 & $\begin{array}{l}\text { o } 0 y \\
\text { A5 }\end{array}$ & $\begin{array}{l}\text { o } 0 y \\
\text { A5 }\end{array}$ & $\begin{array}{l}\text { o } 0 y \\
\text { A5 }\end{array}$ & $\begin{array}{l}\text { o } 0 y \\
\text { A5 }\end{array}$ & $\begin{array}{l}\text { o } 0 y \\
\text { A5 }\end{array}$ & $\begin{array}{l}\text { o } 0 y \\
\text { A5 }\end{array}$ & $\begin{array}{l}\text { o } 0 y \\
\text { A5 }\end{array}$ & $\begin{array}{l}\text { o } 0 y \\
\text { A5 }\end{array}$ & $\begin{array}{l}\text { o } 0 y \\
\text { A5 }\end{array}$ & o 0 y A13 & $\begin{array}{l}\text { o } 0 y \\
\text { A13 }\end{array}$ \\
\hline AEG46 & ALB & 48.4 & 9.43 & $\begin{array}{l}\text { ocy } \\
\text { A5 }\end{array}$ & $\begin{array}{l}\text { ocy } \\
\text { A5 }\end{array}$ & $\begin{array}{l}\text { ocy } \\
\text { A5 }\end{array}$ & $\begin{array}{l}\text { ocy } \\
\text { A5 }\end{array}$ & $\begin{array}{l}\text { ocy } \\
\text { A5 }\end{array}$ & $\begin{array}{l}\text { ocy } \\
\text { A5 }\end{array}$ & $\begin{array}{l}\text { ocy } \\
\text { A5 }\end{array}$ & $\begin{array}{l}\text { ocy } \\
\text { A5 }\end{array}$ & $\begin{array}{l}\text { ocy } \\
\text { A5 }\end{array}$ & $\begin{array}{l}\text { o с у A16/ } \\
\text { A18 }\end{array}$ & $\begin{array}{l}\text { o c y } \\
\text { A16/ } \\
\text { A18 }\end{array}$ \\
\hline AEG47 & ALB & 48.42 & 9.45 & $\begin{array}{l}\text { k } 0 \text { y } \\
\text { A5/A1/ } \\
\text { A6 }\end{array}$ & $\begin{array}{l}\text { k } 0 \text { y } \\
\text { A5/A1/ } \\
\text { A6 }\end{array}$ & $\begin{array}{l}\text { k } 0 y \\
\text { A5/A1/ } \\
\text { A6 }\end{array}$ & $\begin{array}{l}\text { k0y } \\
\text { A5/A1/ } \\
\text { A6 }\end{array}$ & $\begin{array}{l}\text { k } 0 \text { y } \\
\text { A5/A1/ } \\
\text { A6 }\end{array}$ & $\begin{array}{l}\text { k } 0 \text { y } \\
\text { A5/A1/ } \\
\text { A6 }\end{array}$ & $\begin{array}{l}\text { k } 0 y \\
\text { A5/A1/ } \\
\text { A6 }\end{array}$ & $\begin{array}{l}\text { k0 y } \\
\text { A5/A1/ } \\
\text { A6 }\end{array}$ & $\mathrm{k} 0 \mathrm{n} 0$ & k 0 y A19 & $\begin{array}{l}\text { k } 0 y \\
\text { A19 }\end{array}$ \\
\hline AEG48 & ALB & 48.42 & 9.5 & k 0 y 0 & k 0 y 0 & $\mathrm{k} 0$ y 0 & $\mathrm{k} 0$ y 0 & k 0 y 0 & k 0 y 0 & $\mathrm{k} 0$ y 0 & $\mathrm{k} 0$ y 0 & k 0 y 0 & $\begin{array}{l}\text { k } 0 \text { y } \mathrm{A} 15 / \\
\text { A17/A20/ } \\
\text { A19 }\end{array}$ & $\begin{array}{l}\text { k } 0 \text { y } \\
\text { A15/ } \\
\text { A17/ } \\
\text { A20/ } \\
\text { A19 }\end{array}$ \\
\hline AEG49 & ALB & 48.46 & 9.5 & k 0 y 0 & $\mathrm{k} 0$ y 0 & $\mathrm{k} 0$ y 0 & $\mathrm{k} 0$ y 0 & $\mathrm{k} 0$ y 0 & $\mathrm{k} 0$ y 0 & $\mathrm{k} 0$ y 0 & $\mathrm{k} 0$ y 0 & $\mathrm{k} 0$ y 0 & k 0 y A15 & $\begin{array}{l}\mathrm{k} 0 \mathrm{y} \\
\mathrm{A} 15\end{array}$ \\
\hline AEG5 & ALB & 48.4 & 9.44 & k 0 n 0 & $\mathrm{k} 0 \mathrm{n} 0$ & $\mathrm{k} 0 \mathrm{nO}$ & $\mathrm{k} 0 \mathrm{nO}$ & k 0 n 0 & $\mathrm{k} 0 \mathrm{nO}$ & $\mathrm{k} 0 \mathrm{nO}$ & $\mathrm{k} 0 \mathrm{nO}$ & $\mathrm{k} 0 \mathrm{nO}$ & $\mathrm{k} 0 \mathrm{nO}$ & $\mathrm{k} 0 \mathrm{nO}$ \\
\hline AEG50 & ALB & 48.41 & 9.47 & k 0 n 0 & k 0 n 0 & $\mathrm{k} 0 \mathrm{nO}$ & $\mathrm{k} 0 \mathrm{n} 0$ & $\mathrm{k} 0 \mathrm{n} 0$ & $\mathrm{k} 0 \mathrm{n} 0$ & $\mathrm{k} 0 \mathrm{nO}$ & $\mathrm{k} 0 \mathrm{n} 0$ & $\mathrm{k} 0 \mathrm{n} 0$ & $\mathrm{k} 0 \mathrm{n} 0$ & $\mathrm{k} 0 \mathrm{nO}$ \\
\hline AEG6 & ALB & 48.4 & 9.44 & k 0 n 0 & $\mathrm{k} 0 \mathrm{n} 0$ & $\mathrm{k} 0 \mathrm{nO}$ & $\mathrm{k} 0 \mathrm{n} 0$ & $\mathrm{k} 0 \mathrm{n} 0$ & $\mathrm{k} 0 \mathrm{n} 0$ & $\mathrm{k} 0 \mathrm{nO}$ & $\mathrm{k} 0 \mathrm{n} 0$ & $\mathrm{k} 0 \mathrm{n} 0$ & $\mathrm{k} 0 \mathrm{n} 0$ & $\mathrm{k} 0 \mathrm{n} 0$ \\
\hline AEG7 & ALB & 48.39 & 9.38 & k 0 y 0 & k 0 y 0 & $\mathrm{k} 0$ y 0 & $\mathrm{k} 0$ y 0 & k 0 y 0 & $\mathrm{k} 0$ y 0 & $\mathrm{k} 0$ y 0 & $\mathrm{k} 0$ y 0 & $\mathrm{k} 0$ y 0 & $\begin{array}{l}\text { k } 0 \text { y } A 19 / \\
\text { A15 }\end{array}$ & $\begin{array}{l}\text { k0 y } \\
\text { A19/ } \\
\text { A15 }\end{array}$ \\
\hline AEG8 & ALB & 48.42 & 9.49 & $\mathrm{k} 0$ y 0 & k 0 y 0 & $\mathrm{k} 0$ y 0 & $\mathrm{k} 0$ y 0 & k 0 y 0 & $\mathrm{k} 0$ y 0 & $\mathrm{k} 0$ y 0 & $\mathrm{k} 0$ y 0 & $\mathrm{k} 0$ y 0 & $\begin{array}{l}\text { k } 0 \text { y } A 15 / \\
\text { A11 }\end{array}$ & $\begin{array}{l}\text { k } 0 y \\
\text { A15/ } \\
\text { A11 }\end{array}$ \\
\hline AEG9 & ALB & 48.39 & 9.5 & $\mathrm{k} 0$ y 0 & k 0 y 0 & $\mathrm{k} 0$ y 0 & $\mathrm{k} 0$ y 0 & k 0 y 0 & $\mathrm{k} 0$ y 0 & $\mathrm{k} 0$ y 0 & $\mathrm{k} 0$ y 0 & $\mathrm{k} 0$ y 0 & $\begin{array}{l}\text { k } 0 \text { y } \mathrm{A} 15 / \\
\text { A17/A20/ } \\
\text { A19 }\end{array}$ & $\begin{array}{l}\text { k } 0 \text { y } \\
\text { A15/ } \\
\text { A17/ } \\
\text { A20/ } \\
\text { A19 }\end{array}$ \\
\hline HEG1 & $\mathrm{HAl}$ & 50.97 & 10.41 & k 0 n 0 & $\mathrm{k} 0 \mathrm{n} 0$ & $\mathrm{k} 0 \mathrm{nO}$ & $\mathrm{k} 0 \mathrm{nO}$ & $\mathrm{k} 0 \mathrm{n} 0$ & $\mathrm{k} 0 \mathrm{n} 0$ & $\mathrm{k} 0 \mathrm{n} 0$ & $\mathrm{k} 0 \mathrm{n} 0$ & $\mathrm{k} 0 \mathrm{n} 0$ & $\mathrm{k} 0 \mathrm{n} 0$ & $\mathrm{k} 0 \mathrm{n} 0$ \\
\hline HEG10 & $\mathrm{HAl}$ & 51.28 & 10.45 & $\mathrm{k} 0$ y 0 & k 0 y 0 & o 0 y 0 & $\begin{array}{l}\text { o iy } \\
\mathrm{H} 4\end{array}$ & $\begin{array}{l}\text { o iy } \\
\text { H4 }\end{array}$ & o i y H4 & $\begin{array}{l}\text { o a y } \\
\mathrm{H} 4\end{array}$ & $\begin{array}{l}\text { o a y } \\
\mathrm{H} 4\end{array}$ & $\begin{array}{l}\text { o a y } \\
\mathrm{H} 4\end{array}$ & o a y $\mathrm{H} 12$ & $\begin{array}{l}\text { o a y } \\
\text { H12 }\end{array}$ \\
\hline HEG11 & $\mathrm{HAl}$ & 51.28 & 10.46 & $\mathrm{k} 0$ y 0 & k 0 y 0 & o 0 y 0 & $\begin{array}{l}\text { o iy } \\
\mathrm{H} 4\end{array}$ & $\begin{array}{l}\text { o iy } \\
\text { H4 }\end{array}$ & o i y H4 & $\begin{array}{l}\text { o a y } \\
\mathrm{H} 4\end{array}$ & $\begin{array}{l}\text { o a y } \\
\mathrm{H} 4\end{array}$ & $\begin{array}{l}\text { o a y } \\
\text { H4 }\end{array}$ & o a y $\mathrm{H} 12$ & $\begin{array}{l}\text { o a y } \\
\text { H12 }\end{array}$ \\
\hline
\end{tabular}




\begin{tabular}{|c|c|c|c|c|c|c|c|c|c|c|c|c|c|c|}
\hline EP_Plot_ID & Explo & Latitude & Longitude & 2006 & 2007 & 2008 & 2009 & 2010 & 2011 & 2012 & 2013 & 2014 & 2015 & 2016 \\
\hline HEG12 & $\mathrm{HAl}$ & 51.08 & 10.58 & $\mathrm{k} 0$ y 0 & $\mathrm{k} 0$ y 0 & k 0 y 0 & $\begin{array}{l}\text { kOy } \\
\mathrm{H} 4\end{array}$ & $\begin{array}{l}\text { k } 0 \text { y } \\
\text { H5 }\end{array}$ & $\begin{array}{l}\text { k0y } \\
\text { H5 }\end{array}$ & $\begin{array}{l}\text { k0y } \\
\text { H5 }\end{array}$ & $\begin{array}{l}\mathrm{kOy} \\
\mathrm{H} 5\end{array}$ & $\begin{array}{l}\text { k } 0 y \\
\text { H5 }\end{array}$ & k 0 y H13 & $\mathrm{k} 0 \mathrm{nO}$ \\
\hline HEG13 & $\mathrm{HAl}$ & 51.26 & 10.38 & $\mathrm{k} 0$ y 0 & $\mathrm{k} 0$ y 0 & k 0 y 0 & $\mathrm{k} 0$ y 0 & $\mathrm{k} 0$ y 0 & $\mathrm{k} 0$ y 0 & $\mathrm{k} 0$ y 0 & k 0 y 0 & $\mathrm{k} 0$ y 0 & $\mathrm{k} 0 \mathrm{n} 0$ & $\mathrm{k} 0$ y 0 \\
\hline HEG14 & $\mathrm{HAl}$ & 51.29 & 10.44 & $\mathrm{k} 0$ y 0 & $\mathrm{k} 0$ y 0 & k 0 y 0 & $\begin{array}{l}\text { k } 0 \text { y } \\
\mathrm{H} 4\end{array}$ & $\mathrm{k} 0$ y 0 & $\begin{array}{l}\text { k } 0 \text { y } \\
\text { H5 }\end{array}$ & $\begin{array}{l}\text { k } 0 \text { y } \\
\text { H5 }\end{array}$ & $\begin{array}{l}\text { k } 0 \text { y } \\
\text { H5 }\end{array}$ & $\begin{array}{l}\text { k } 0 \text { y } \\
\text { H5 }\end{array}$ & k 0 y H13 & $\begin{array}{l}\text { k 0 y } \\
\text { H13 }\end{array}$ \\
\hline HEG15 & $\mathrm{HAl}$ & 51.07 & 10.49 & $\begin{array}{l}\text { k } 0 \text { y } \\
\mathrm{H} 2\end{array}$ & $\begin{array}{l}\text { k } 0 y \\
\text { H2 }\end{array}$ & $\begin{array}{l}\text { k } 0 \text { y } \\
\mathrm{H} 2\end{array}$ & $\mathrm{k} 0 \mathrm{n} 0$ & $\mathrm{k} 0 \mathrm{n} 0$ & $\mathrm{k} 0 \mathrm{n} 0$ & $\mathrm{k} 0$ y 0 & $\mathrm{k} 0$ y 0 & $\mathrm{k} 0$ y 0 & $\mathrm{k} 0$ y 0 & $\mathrm{k} 0$ y 0 \\
\hline HEG16 & $\mathrm{HAl}$ & 51.03 & 10.46 & $\begin{array}{l}\text { k } 0 y \\
\text { H3 }\end{array}$ & $\begin{array}{l}\text { k } 0 y \\
\text { H3 }\end{array}$ & $\begin{array}{l}\text { k O y } \\
\text { H3 }\end{array}$ & $\begin{array}{l}\text { k O y } \\
\text { H8 }\end{array}$ & $\begin{array}{l}\text { k O y } \\
\text { H8 }\end{array}$ & $\begin{array}{l}\text { k } 0 y \\
\text { H8 }\end{array}$ & $\begin{array}{l}\text { k Oy } \\
\text { H8 }\end{array}$ & $\begin{array}{l}\text { k O y } \\
\text { H8 }\end{array}$ & $\begin{array}{l}\text { k Oy } \\
\text { H8 }\end{array}$ & k 0 y H16 & $\begin{array}{l}\text { k } 0 y \\
\text { H16 }\end{array}$ \\
\hline HEG17 & $\mathrm{HAl}$ & 51.07 & 10.47 & $\mathrm{k} 0$ y 0 & k 0 y 0 & k 0 y 0 & $\begin{array}{l}\text { k } 0 \text { y } \\
\mathrm{H} 8\end{array}$ & $\begin{array}{l}\text { k } 0 \text { y } \\
\mathrm{H} 8\end{array}$ & $\begin{array}{l}\text { k } 0 \text { y } \\
\text { H8 }\end{array}$ & $\begin{array}{l}\text { k } 0 \text { y } \\
\text { H8 }\end{array}$ & $\begin{array}{l}\text { k } 0 \text { y } \\
\mathrm{H} 8\end{array}$ & $\begin{array}{l}\text { k } 0 \text { y } \\
\text { H8 }\end{array}$ & k 0 y H16 & $\begin{array}{l}\text { k 0 y } \\
\text { H17 }\end{array}$ \\
\hline HEG18 & $\mathrm{HAl}$ & 51.28 & 10.42 & $\mathrm{k} 0$ y 0 & $\mathrm{k} 0$ y 0 & $\mathrm{k} 0$ y 0 & $\mathrm{k} 0$ y 7 & $\mathrm{k} 0$ y 0 & $\begin{array}{l}\text { k O y } \\
\text { H8 }\end{array}$ & $\begin{array}{l}\text { k O y } \\
\text { H8 }\end{array}$ & $\begin{array}{l}\text { k O y } \\
\mathrm{H} 8\end{array}$ & $\begin{array}{l}\text { k O y } \\
\text { H8 }\end{array}$ & k 0 y H16 & $\begin{array}{l}\text { k } 0 \text { y } \\
\text { H16 }\end{array}$ \\
\hline HEG19 & $\mathrm{HAl}$ & 51.07 & 10.47 & $\mathrm{k} 0$ y 0 & $\mathrm{k} 0$ y 0 & $\mathrm{k} 0$ y 0 & $\begin{array}{l}\text { k O y } \\
\text { H8 }\end{array}$ & $\mathrm{k} 0$ y 0 & $\begin{array}{l}\text { k } 0 y \\
\text { H8 }\end{array}$ & $\begin{array}{l}\text { k O y } \\
\text { H8 }\end{array}$ & $\begin{array}{l}\mathrm{kOy} \\
\mathrm{H} 8\end{array}$ & $\begin{array}{l}\text { k O y } \\
\text { H8 }\end{array}$ & k 0 y H16 & $\begin{array}{l}\text { k } 0 y \\
\mathrm{H} 17\end{array}$ \\
\hline HEG2 & $\mathrm{HAl}$ & 51 & 10.43 & $\mathrm{k} 0 \mathrm{n} 0$ & $\mathrm{k} 0 \mathrm{nO}$ & k 0 n 0 & $\mathrm{k} 0 \mathrm{n} 0$ & $\mathrm{k} 0 \mathrm{n} 0$ & k $0 \mathrm{n} 0$ & $\mathrm{k} 0$ y 0 & $\mathrm{k} 0 \mathrm{n} 0$ & $\mathrm{k} 0 \mathrm{n} 0$ & k 0 n 0 & $\mathrm{k} 0 \mathrm{nO}$ \\
\hline HEG20 & $\mathrm{HAl}$ & 51.22 & 10.37 & $\mathrm{k} 0$ y 0 & $\mathrm{k} 0$ y 0 & k 0 y 0 & $\mathrm{k} 0$ y 0 & $\mathrm{k} 0$ y 0 & $\begin{array}{l}\text { k } 0 \text { y } \\
\text { H8 }\end{array}$ & $\begin{array}{l}\text { k O y } \\
\text { H8 }\end{array}$ & $\begin{array}{l}\text { kOy } \\
\mathrm{H} 9\end{array}$ & $\begin{array}{l}\text { k O y } \\
\text { H8 }\end{array}$ & k 0 y H16 & $\begin{array}{l}\text { k 0 y } \\
\text { H16 }\end{array}$ \\
\hline HEG21 & $\mathrm{HAl}$ & 51.19 & 10.75 & $\mathrm{k} 0$ y 0 & $\mathrm{k} 0$ y 0 & k 0 y 0 & $\begin{array}{l}\text { k O y } \\
\text { H8 }\end{array}$ & $\begin{array}{l}\text { k } 0 \text { y } \\
\text { H8 }\end{array}$ & $\begin{array}{l}\text { k } 0 \text { y } \\
\text { H8 }\end{array}$ & $\begin{array}{l}\text { k O y } \\
\text { H8 }\end{array}$ & $\begin{array}{l}\text { k } 0 \text { y } \\
\text { H8 }\end{array}$ & $\begin{array}{l}\text { k O y } \\
\text { H8 }\end{array}$ & k 0 y H16 & $\begin{array}{l}\text { k 0 y } \\
\text { H16 }\end{array}$ \\
\hline HEG22 & $\mathrm{HAl}$ & 51.03 & 10.32 & k 0 y 0 & $\mathrm{k} 0$ y 0 & k 0 y 0 & $\begin{array}{l}\text { kO y } \\
\text { H9 }\end{array}$ & $\begin{array}{l}\text { k } 0 \text { y } \\
\text { H9 }\end{array}$ & $\begin{array}{l}\text { k } 0 \text { y } \\
\text { H9 }\end{array}$ & $\begin{array}{l}\text { k } 0 \text { y } \\
\text { H9 }\end{array}$ & $\begin{array}{l}\text { k } 0 \text { y } \\
\text { H9 }\end{array}$ & $\begin{array}{l}\text { k O y } \\
\text { H9 }\end{array}$ & k 0 y H13 & $\mathrm{k} 0$ y 0 \\
\hline HEG23 & $\mathrm{HAl}$ & 51.13 & 10.34 & $\begin{array}{l}\text { o } 0 y \\
\mathrm{H} 1\end{array}$ & $\begin{array}{l}\text { o } 0 y \\
\mathrm{H} 1\end{array}$ & $\begin{array}{l}\text { o } 0 y \\
\text { H1 }\end{array}$ & $\begin{array}{l}\text { o i y } \\
\text { H4/ } \\
\text { H10 }\end{array}$ & o 0 y 0 & $\begin{array}{l}\text { o iy } \\
\text { H10 }\end{array}$ & $\begin{array}{l}\text { o iy } \\
\text { H10 }\end{array}$ & $\begin{array}{l}\text { o iy } \\
\text { H10 }\end{array}$ & $\begin{array}{l}\text { o iy } \\
\text { H10 }\end{array}$ & o iy H12 & $\begin{array}{l}\text { o a y } \\
\text { H12 }\end{array}$ \\
\hline HEG24 & HAI & 51.1 & 10.35 & $\begin{array}{l}\text { o } 0 y \\
\text { H1 }\end{array}$ & $\begin{array}{l}\text { o } 0 y \\
\text { H1 }\end{array}$ & $\begin{array}{l}\text { o } 0 y \\
\text { H1 }\end{array}$ & $\begin{array}{l}\text { o i y } \\
\text { H4/ } \\
\text { H10 }\end{array}$ & o 0 y 0 & $\begin{array}{l}\text { o iy } \\
\text { H10 }\end{array}$ & $\begin{array}{l}\text { o iy } \\
\text { H10 }\end{array}$ & $\begin{array}{l}\text { o iy } \\
\text { H10 }\end{array}$ & $\begin{array}{l}\text { o iy } \\
\text { H10 }\end{array}$ & o a y $\mathrm{H} 12$ & $\begin{array}{l}\text { o a y } \\
\text { H12 }\end{array}$ \\
\hline HEG25 & $\mathrm{HAl}$ & 51.02 & 10.32 & $\mathrm{k} 0$ y 0 & $\mathrm{k} 0$ y 0 & k 0 y 0 & $\begin{array}{l}\text { kO y } \\
\text { H9 }\end{array}$ & $\begin{array}{l}\text { k O y } \\
\text { H9 }\end{array}$ & $\begin{array}{l}\text { k O y } \\
\text { H9 }\end{array}$ & $\begin{array}{l}\text { k } 0 y \\
\text { H9 }\end{array}$ & $\begin{array}{l}\text { k O y } \\
\mathrm{H} 9\end{array}$ & $\begin{array}{l}\text { k O y } \\
\text { H9 }\end{array}$ & k 0 y H13 & $\mathrm{k} 0$ y 0 \\
\hline HEG26 & $\mathrm{HAl}$ & 51.28 & 10.37 & $\mathrm{k} 0$ y 0 & o 0 y 0 & o 0 y 0 & $\begin{array}{l}\text { o i y } \\
\text { H4 }\end{array}$ & $\begin{array}{l}\text { o iy } \\
\text { H4 }\end{array}$ & o i y H4 & $\begin{array}{l}\text { o a y } \\
\mathrm{H} 4\end{array}$ & $\begin{array}{l}\text { o a y } \\
\mathrm{H} 4\end{array}$ & $\begin{array}{l}\text { o a y } \\
\mathrm{H} 4\end{array}$ & o a y $\mathrm{H} 12$ & $\begin{array}{l}\text { o a y } \\
\text { H12 }\end{array}$ \\
\hline HEG27 & $\mathrm{HAl}$ & 51.09 & 10.6 & $\mathrm{k} 0$ y 0 & $\mathrm{k} 0$ y 0 & k 0 y 0 & $\begin{array}{l}\text { k0y } \\
\text { H5 }\end{array}$ & $\begin{array}{l}\text { k } 0 y \\
\text { H5 }\end{array}$ & $\begin{array}{l}\text { k0y } \\
\text { H5 }\end{array}$ & $\begin{array}{l}\text { k O y } \\
\text { H5 }\end{array}$ & $\begin{array}{l}\text { k O y } \\
\text { H5 }\end{array}$ & $\begin{array}{l}\text { k } 0 y \\
\text { H5 }\end{array}$ & $\mathrm{k} \mathrm{O}$ y H13 & $\mathrm{k} 0 \mathrm{nO}$ \\
\hline HEG28 & $\mathrm{HAl}$ & 51.27 & 10.5 & $\begin{array}{l}\text { k } 0 \text { y } \\
\text { H2 }\end{array}$ & $\mathrm{k} 0 \mathrm{nO}$ & $\mathrm{k} 0 \mathrm{n} 0$ & $\mathrm{k} 0 \mathrm{n} 0$ & $\mathrm{k} 0 \mathrm{n} 0$ & $\mathrm{k} 0 \mathrm{nO}$ & $\begin{array}{l}\text { k } 0 \text { y } \\
\mathrm{H} 2\end{array}$ & $\begin{array}{l}\text { k } 0 \text { y } \\
\mathrm{H} 4\end{array}$ & $\begin{array}{l}\text { k a y } \\
\mathrm{H} 4\end{array}$ & o a y $\mathrm{H} 12$ & $\begin{array}{l}\text { o a y } \\
\text { H12 }\end{array}$ \\
\hline
\end{tabular}




\begin{tabular}{|c|c|c|c|c|c|c|c|c|c|c|c|c|c|c|}
\hline EP_Plot_ID & Explo & Latitude & Longitude & 2006 & 2007 & 2008 & 2009 & 2010 & 2011 & 2012 & 2013 & 2014 & 2015 & 2016 \\
\hline HEG29 & HAI & 51.26 & 10.5 & $\begin{array}{l}\mathrm{k} 0 \mathrm{y} \\
\mathrm{H} 2\end{array}$ & $\mathrm{k} 0 \mathrm{n} 0$ & $\mathrm{k} 0 \mathrm{n} 0$ & $\mathrm{k} 0 \mathrm{n} 0$ & $\mathrm{k} 0 \mathrm{n} 0$ & $\mathrm{k} 0 \mathrm{n} 0$ & $\begin{array}{l}\mathrm{k} 0 \mathrm{y} \\
\mathrm{H} 2\end{array}$ & $\begin{array}{l}\mathrm{k} 0 \mathrm{y} \\
\mathrm{H} 4\end{array}$ & $\begin{array}{l}\text { kay } \\
\mathrm{H} 4\end{array}$ & o а y $\mathrm{H} 12$ & $\begin{array}{l}\text { o a y } \\
\text { H12 }\end{array}$ \\
\hline HEG3 & $\mathrm{HAl}$ & 51 & 10.43 & $\mathrm{k} 0 \mathrm{n} 0$ & $\mathrm{k} 0 \mathrm{nO}$ & $\mathrm{k} 0 \mathrm{n} 0$ & $\mathrm{k} 0 \mathrm{n} 0$ & $\mathrm{k} 0 \mathrm{n} 0$ & $\mathrm{k} 0 \mathrm{n} 0$ & $\mathrm{k} 0 \mathrm{n} 0$ & k 0 n 0 & $\mathrm{k} 0 \mathrm{n} 0$ & $\mathrm{k} 0 \mathrm{nO}$ & $\mathrm{k} 0 \mathrm{nO}$ \\
\hline HEG30 & $\mathrm{HAl}$ & 51.2 & 10.36 & $\begin{array}{l}\text { k } 0 y \\
\mathrm{H} 2\end{array}$ & $\begin{array}{l}\text { k } 0 \text { y } \\
\mathrm{H} 2\end{array}$ & $\begin{array}{l}\text { k } 0 \text { y } \\
\text { H2 }\end{array}$ & $\begin{array}{l}\text { k } 0 \text { y } \\
\text { H5 }\end{array}$ & $\begin{array}{l}\text { k } 0 \text { y } \\
\text { H5 }\end{array}$ & $\begin{array}{l}\text { k } 0 \text { y } \\
\text { H5 }\end{array}$ & $\begin{array}{l}\text { k } 0 \text { y } \\
\text { H5 }\end{array}$ & $\begin{array}{l}\text { k } 0 \text { y } \\
\text { H5 }\end{array}$ & $\begin{array}{l}\text { k0 y } \\
\text { H5 }\end{array}$ & k 0 y H13 & $\begin{array}{l}\text { k0 y } \\
\text { H13 }\end{array}$ \\
\hline HEG31 & $\mathrm{HAl}$ & 51.17 & 10.22 & $\mathrm{k} 0$ y 0 & $\begin{array}{l}\text { k } 0 \text { y } \\
\mathrm{H} 4\end{array}$ & $\begin{array}{l}\mathrm{k} 0 \mathrm{y} \\
\mathrm{H} 4\end{array}$ & $\begin{array}{l}\text { k0y } \\
\text { H5 }\end{array}$ & $\begin{array}{l}\text { k } 0 \text { y } \\
\text { H5 }\end{array}$ & $\begin{array}{l}\text { k0y } \\
\text { H5 }\end{array}$ & $\begin{array}{l}\text { k0y } \\
\text { H5 }\end{array}$ & $\begin{array}{l}\mathrm{k} 0 \mathrm{y} \\
\mathrm{H} 5\end{array}$ & $\begin{array}{l}\text { k } 0 \text { y } \\
\text { H5 }\end{array}$ & k 0 y H15 & $\begin{array}{l}\text { k0y } \\
\text { H15 }\end{array}$ \\
\hline HEG32 & $\mathrm{HAl}$ & 51.08 & 10.57 & k 0 y 0 & k 0 y 0 & $\mathrm{k} 0$ y 0 & $\begin{array}{l}\text { k } 0 y \\
\text { H5 }\end{array}$ & $\begin{array}{l}\mathrm{k} 0 \mathrm{y} \\
\mathrm{H} 5\end{array}$ & $\begin{array}{l}\text { k0y } \\
\text { H5 }\end{array}$ & $\begin{array}{l}\text { k0y } \\
\text { H5 }\end{array}$ & $\begin{array}{l}\text { k } 0 y \\
\text { H5 }\end{array}$ & $\begin{array}{l}\text { k } 0 \text { y } \\
\text { H5 }\end{array}$ & k 0 y H13 & $\mathrm{k} 0 \mathrm{no}$ \\
\hline HEG33 & $\mathrm{HAl}$ & 51.11 & 10.43 & $\begin{array}{l}\text { k } 0 \text { y } \\
\mathrm{H} 2 / \\
\mathrm{H} 18\end{array}$ & $\mathrm{k} 0 \mathrm{n} 0$ & $\mathrm{k} 0 \mathrm{n} 0$ & $\mathrm{k} 0 \mathrm{n} 0$ & $\mathrm{k} 0 \mathrm{n} 0$ & k 0 y 0 & $\begin{array}{l}\text { k } 0 y \\
H 2\end{array}$ & $\begin{array}{l}\text { k } 0 \text { y } \\
\mathrm{H} 4\end{array}$ & $\begin{array}{l}\text { ka y } \\
\mathrm{H} 4\end{array}$ & o a y $\mathrm{H} 12$ & $\begin{array}{l}\text { o a y } \\
\text { H12 }\end{array}$ \\
\hline HEG34 & $\mathrm{HAl}$ & 51.21 & 10.39 & $\begin{array}{l}\text { ogy } \\
\text { H1/ } \\
\text { H18 }\end{array}$ & $\begin{array}{l}\text { ogy } \\
\text { H1/ } \\
\text { H18 }\end{array}$ & $\begin{array}{l}\text { og y } \\
\text { H1/ } \\
\text { H18 }\end{array}$ & $\begin{array}{l}\text { ogy } \\
\mathrm{H} 4\end{array}$ & og y 0 & $\begin{array}{l}\text { ogy } \\
\text { H1/H18 }\end{array}$ & $\begin{array}{l}\text { ogy } \\
\text { H1/H18 }\end{array}$ & $\begin{array}{l}\text { og y } \\
\mathrm{H} 4\end{array}$ & $\begin{array}{l}\text { ogy } \\
\mathrm{H} 4\end{array}$ & o g y H12 & $\begin{array}{l}\text { ogy } \\
\text { H12 }\end{array}$ \\
\hline HEG35 & $\mathrm{HAl}$ & 51.22 & 10.41 & $\begin{array}{l}\text { og y } \\
\text { H1/ } \\
\text { H18 }\end{array}$ & $\begin{array}{l}\text { ogy } \\
\text { H1/ } \\
\text { H18 }\end{array}$ & $\begin{array}{l}\text { og y } \\
\text { H1/ } \\
\text { H18 }\end{array}$ & $\begin{array}{l}\text { ogy } \\
\mathrm{H} 4\end{array}$ & og y 0 & $\begin{array}{l}\text { ogy } \\
\text { H1/H18 }\end{array}$ & $\begin{array}{l}\text { ogy } \\
\mathrm{H} 4\end{array}$ & $\begin{array}{l}\text { ogy } \\
\mathrm{H} 4\end{array}$ & $\begin{array}{l}\text { ogy } \\
\mathrm{H} 4\end{array}$ & og y H12 & $\begin{array}{l}\text { ogy } \\
\text { H12 }\end{array}$ \\
\hline HEG36 & $\mathrm{HAl}$ & 51.03 & 10.51 & $\begin{array}{l}\mathrm{k} 0 \mathrm{y} \\
\mathrm{H} 2\end{array}$ & $\begin{array}{l}\mathrm{k} 0 \mathrm{y} \\
\mathrm{H} 2\end{array}$ & $\begin{array}{l}\mathrm{k} 0 \mathrm{y} \\
\mathrm{H} 2\end{array}$ & $\mathrm{k} 0 \mathrm{n} 0$ & k 0 y 0 & $\mathrm{k} 0 \mathrm{n} 0$ & k 0 y 0 & k 0 y 0 & k 0 y 0 & k 0 y H14 & $\begin{array}{l}\text { k } 0 \text { y } \\
\text { H14 }\end{array}$ \\
\hline HEG37 & $\mathrm{HAl}$ & 51.03 & 10.51 & $\begin{array}{l}\mathrm{k} 0 \mathrm{y} \\
\mathrm{H} 2\end{array}$ & $\begin{array}{l}\mathrm{k} 0 \mathrm{y} \\
\mathrm{H} 2\end{array}$ & $\begin{array}{l}\mathrm{k} 0 \mathrm{y} \\
\mathrm{H} 2\end{array}$ & $\mathrm{k} 0 \mathrm{n} 0$ & k 0 y 0 & $\mathrm{k} 0 \mathrm{n} 0$ & k 0 y 0 & k 0 y 0 & $\mathrm{k} 0$ y 0 & k 0 y 0 & k 0 y 0 \\
\hline HEG38 & $\mathrm{HAI}$ & 51.12 & 10.34 & $\begin{array}{l}\text { o } 0 y \\
\mathrm{H} 1\end{array}$ & $\begin{array}{l}\text { o } 0 y \\
\mathrm{H} 1\end{array}$ & $\begin{array}{l}\text { o } 0 y \\
\mathrm{H} 1\end{array}$ & $\begin{array}{l}\text { OO y } \\
\mathrm{H} 6\end{array}$ & o 0 y 0 & $\begin{array}{l}\text { k } 0 \text { y } \\
\mathrm{H} 7\end{array}$ & $\begin{array}{l}\text { k } 0 y \\
H 7\end{array}$ & $\begin{array}{l}\text { o } 0 y \\
\mathrm{H} 7\end{array}$ & $\begin{array}{l}\text { OO y } \\
\mathrm{H} 7\end{array}$ & o a y $\mathrm{H} 12$ & $\begin{array}{l}\text { o a y } \\
\text { H12 }\end{array}$ \\
\hline HEG39 & $\mathrm{HAl}$ & 51.12 & 10.35 & $\begin{array}{l}\text { o } 0 y \\
\mathrm{H} 1\end{array}$ & $\begin{array}{l}\text { o } 0 y \\
\mathrm{H} 1\end{array}$ & $\begin{array}{l}\text { o } 0 y \\
\mathrm{H} 1\end{array}$ & $\begin{array}{l}\text { oiy } \\
\text { H4/H6 }\end{array}$ & o 0 y 0 & o iу H6 & o i y H6 & o iу $\mathrm{H} 6$ & оіун6 & o iy H12 & $\begin{array}{l}\text { oiy } \\
\text { H12 }\end{array}$ \\
\hline HEG4 & $\mathrm{HAl}$ & 51.11 & 10.44 & $\begin{array}{l}\mathrm{k} 0 \mathrm{y} \\
\mathrm{H} 2\end{array}$ & $\mathrm{k} 0 \mathrm{n} 0$ & $\mathrm{k} 0 \mathrm{n} 0$ & $\mathrm{k} 0 \mathrm{n} 0$ & $\mathrm{k} 0 \mathrm{n} 0$ & $\mathrm{k} 0 \mathrm{n} 0$ & $\mathrm{k} 0 \mathrm{n} 0$ & $\mathrm{k} 0 \mathrm{n} 0$ & $\mathrm{k} 0 \mathrm{nO}$ & $\mathrm{k} 0 \mathrm{n} 0$ & k 0 y 0 \\
\hline HEG40 & $\mathrm{HAl}$ & 50.97 & 10.45 & k 0 y 0 & k 0 y 0 & k 0 y 0 & $\begin{array}{l}\text { k } 0 \text { y } \\
\mathrm{H} 7\end{array}$ & $\begin{array}{l}\text { k } 0 y \\
H 7\end{array}$ & $\begin{array}{l}\text { k0y } \\
\mathrm{H} 7\end{array}$ & $\begin{array}{l}\text { k } 0 \text { y } \\
\mathrm{H} 7\end{array}$ & $\begin{array}{l}\text { k } 0 y \\
H 7\end{array}$ & $\begin{array}{l}\text { k } 0 \text { y } \\
\mathrm{H} 7\end{array}$ & k 0 y H13 & $\begin{array}{l}\text { k 0 y } \\
\text { H14 }\end{array}$ \\
\hline HEG41 & $\mathrm{HAl}$ & 51.22 & 10.37 & k 0 y 0 & k 0 y 0 & k 0 y 0 & k 0 y 0 & k 0 y 0 & k 0 y 0 & k 0 y 0 & $\begin{array}{l}\text { k Oy } \\
\text { H9 }\end{array}$ & $\begin{array}{l}\text { k O y } \\
\text { H8 }\end{array}$ & k 0 y H16 & $\begin{array}{l}\text { k0y } \\
\text { H16 }\end{array}$ \\
\hline HEG42 & $\mathrm{HAl}$ & 51.07 & 10.46 & k 0 y 0 & k 0 y 0 & $\mathrm{k} 0$ y 0 & $\begin{array}{l}\mathrm{k} 0 \mathrm{y} \\
\mathrm{H} 8\end{array}$ & k 0 y 0 & $\begin{array}{l}\mathrm{k} 0 \mathrm{y} \\
\mathrm{H} 8\end{array}$ & $\begin{array}{l}\mathrm{k} 0 \mathrm{y} \\
\mathrm{H} 8\end{array}$ & $\begin{array}{l}\mathrm{k} 0 \mathrm{y} \\
\mathrm{H} 8\end{array}$ & $\begin{array}{l}\text { k } 0 \text { y } \\
\text { H8 }\end{array}$ & $\mathrm{k} 0$ y H17 & $\begin{array}{l}\text { k 0 y } \\
\text { H17 }\end{array}$ \\
\hline HEG43 & $\mathrm{HAl}$ & 51.3 & 10.44 & $\mathrm{k} 0$ y 0 & k 0 y 0 & k 0 y 0 & $\begin{array}{l}\text { k O y } \\
\mathrm{H} 6\end{array}$ & k 0 y 0 & $\begin{array}{l}\text { k O y } \\
\text { H8 }\end{array}$ & $\begin{array}{l}\text { k } 0 \text { y } \\
\text { H8 }\end{array}$ & $\begin{array}{l}\text { k } 0 \mathrm{y} \\
\mathrm{H} 8\end{array}$ & $\begin{array}{l}\text { k O y } \\
\text { H8 }\end{array}$ & $\mathrm{k} 0 \mathrm{yH} 16$ & $\begin{array}{l}\text { k } 0 y \\
\text { H16 }\end{array}$ \\
\hline
\end{tabular}




\begin{tabular}{|c|c|c|c|c|c|c|c|c|c|c|c|c|c|c|}
\hline EP_Plot_ID & Explo & Latitude & Longitude & 2006 & 2007 & 2008 & 2009 & 2010 & 2011 & 2012 & 2013 & 2014 & 2015 & 2016 \\
\hline HEG44 & $\mathrm{HAl}$ & 51.06 & 10.48 & $\begin{array}{l}\text { kOy } \\
\mathrm{H} 3\end{array}$ & $\begin{array}{l}\text { k } 0 y \\
\text { H3 }\end{array}$ & $\begin{array}{l}\text { k0y } \\
\text { H3 }\end{array}$ & $\begin{array}{l}\text { k O y } \\
\text { H8 }\end{array}$ & $\begin{array}{l}\text { k } 0 \text { y } \\
\text { H8 }\end{array}$ & $\begin{array}{l}\text { k } 0 y \\
\text { H8 }\end{array}$ & $\begin{array}{l}\text { k O y } \\
\text { H8 }\end{array}$ & $\begin{array}{l}\text { k } 0 y \\
\text { H8 }\end{array}$ & $\begin{array}{l}\text { k } 0 y \\
\text { H8 }\end{array}$ & k 0 y H16 & $\begin{array}{l}\text { k } 0 y \\
\text { H16 }\end{array}$ \\
\hline HEG45 & $\mathrm{HAl}$ & 51.04 & 10.51 & $\begin{array}{l}\text { kOy } \\
\mathrm{H} 3\end{array}$ & $\begin{array}{l}\text { k } 0 y \\
\text { H3 }\end{array}$ & $\begin{array}{l}\text { k O y } \\
\text { H3 }\end{array}$ & $\mathrm{k} 0 \mathrm{n} 0$ & $\begin{array}{l}\text { k } 0 y \\
\text { H11 }\end{array}$ & $\begin{array}{l}\text { k } 0 y \\
\text { H8 }\end{array}$ & $\begin{array}{l}\text { k O y } \\
\text { H8 }\end{array}$ & $\begin{array}{l}\text { k } 0 y \\
\text { H8 }\end{array}$ & $\begin{array}{l}\text { k } 0 \text { y } \\
\text { H8 }\end{array}$ & k 0 y H16 & $\begin{array}{l}\text { k0 y } \\
\text { H16 }\end{array}$ \\
\hline HEG46 & $\mathrm{HAl}$ & 51.21 & 10.75 & $\mathrm{k} 0$ y 0 & $\mathrm{k} 0$ y 0 & k 0 y 0 & $\begin{array}{l}\text { k } 0 \text { y } \\
\mathrm{H} 8\end{array}$ & $\begin{array}{l}\text { k } 0 \text { y } \\
\text { H8 }\end{array}$ & $\begin{array}{l}\text { k } 0 \text { y } \\
\text { H8 }\end{array}$ & $\begin{array}{l}\text { k } 0 \text { y } \\
\text { H8 }\end{array}$ & $\begin{array}{l}\text { k } 0 y \\
\text { H8 }\end{array}$ & $\begin{array}{l}\text { k } 0 \text { y } \\
\mathrm{H} 8\end{array}$ & k 0 у H16 & $\begin{array}{l}\text { k 0 y } \\
\text { H16 }\end{array}$ \\
\hline HEG47 & $\mathrm{HAl}$ & 51.28 & 10.37 & k 0 y 0 & k 0 y 0 & o 0 y 0 & $\begin{array}{l}\text { o iy } \\
\mathrm{H} 4\end{array}$ & $\begin{array}{l}\text { o iy } \\
\text { H4 }\end{array}$ & o i y 44 & $\begin{array}{l}\text { o a y } \\
\mathrm{H} 4\end{array}$ & $\begin{array}{l}\text { o a y } \\
\mathrm{H} 4\end{array}$ & $\begin{array}{l}\text { o a y } \\
\mathrm{H} 4\end{array}$ & o a y H12 & $\begin{array}{l}\text { o a y } \\
\text { H12 }\end{array}$ \\
\hline HEG48 & $\mathrm{HAl}$ & 51.29 & 10.38 & $\mathrm{k} 0$ y 0 & k 0 y 0 & o 0 y 0 & $\begin{array}{l}\text { o iy } \\
\mathrm{H} 4\end{array}$ & $\begin{array}{l}\text { o iy } \\
\text { H4 }\end{array}$ & o i y H4 & $\begin{array}{l}\text { o a y } \\
\mathrm{H} 4\end{array}$ & $\begin{array}{l}\text { o a y } \\
\mathrm{H} 4\end{array}$ & $\begin{array}{l}\text { o a y } \\
\mathrm{H} 4\end{array}$ & o a y H12 & $\begin{array}{l}\text { o a y } \\
\text { H12 }\end{array}$ \\
\hline HEG49 & $\mathrm{HAl}$ & 51.28 & 10.39 & $\mathrm{k} 0$ y 0 & $\mathrm{k} 0$ y 0 & o 0 y 0 & $\begin{array}{l}\text { o i y } \\
\text { H4 }\end{array}$ & $\begin{array}{l}\text { o iy } \\
\text { H4 }\end{array}$ & o iy 44 & $\begin{array}{l}\text { o a y } \\
\mathrm{H} 4\end{array}$ & $\begin{array}{l}\text { o a y } \\
\mathrm{H} 4\end{array}$ & $\begin{array}{l}\text { o a y } \\
\mathrm{H} 4\end{array}$ & o a y H12 & $\begin{array}{l}\text { o a y } \\
\text { H12 }\end{array}$ \\
\hline HEG5 & $\mathrm{HAl}$ & 51.22 & 10.32 & $\begin{array}{l}\text { k } 0 \text { y } \\
\mathrm{H} 2\end{array}$ & $\begin{array}{l}\text { k } 0 y \\
H 2\end{array}$ & $\begin{array}{l}\text { k } 0 \text { y } \\
\text { H2 }\end{array}$ & $\begin{array}{l}\text { k } 0 \text { y } \\
\text { H5 }\end{array}$ & $\begin{array}{l}\text { k } 0 \text { y } \\
\text { H5 }\end{array}$ & $\begin{array}{l}\text { k0y } \\
\text { H5 }\end{array}$ & $\begin{array}{l}\text { k } 0 \text { y } \\
\text { H5 }\end{array}$ & $\begin{array}{l}\text { k } 0 \text { y } \\
\text { H5 }\end{array}$ & $\begin{array}{l}\text { k } 0 \text { y } \\
\text { H5 }\end{array}$ & k 0 y H13 & $\begin{array}{l}\text { k0y } \\
\text { H13 }\end{array}$ \\
\hline HEG50 & $\mathrm{HAl}$ & 51.28 & 10.42 & k 0 y 0 & $\mathrm{k} 0$ y 0 & k 0 y 0 & $\begin{array}{l}\text { o iy } \\
\mathrm{H} 4\end{array}$ & $\begin{array}{l}\text { o i y } \\
\text { H4 }\end{array}$ & o i y 44 & $\begin{array}{l}\text { o a y } \\
\mathrm{H} 4\end{array}$ & $\begin{array}{l}\text { o a y } \\
\mathrm{H} 4\end{array}$ & $\begin{array}{l}\text { o a y } \\
\mathrm{H} 4\end{array}$ & o a y H12 & $\begin{array}{l}\text { o a y } \\
\text { H12 }\end{array}$ \\
\hline HEG6 & $\mathrm{HAl}$ & 51.21 & 10.39 & $\begin{array}{l}\text { og y } \\
\mathrm{H} 1 / \\
\mathrm{H} 18\end{array}$ & $\begin{array}{l}\text { ogy } \\
\text { H1/ } \\
\text { H18 }\end{array}$ & $\begin{array}{l}\text { ogy } \\
\text { H1/ } \\
\text { H18 }\end{array}$ & $\begin{array}{l}\text { ogy } \\
\mathrm{H} 4\end{array}$ & og y 0 & $\begin{array}{l}\text { o g y } \\
\text { H4/H18 }\end{array}$ & $\begin{array}{l}\text { ogy } \\
\mathrm{H} 4\end{array}$ & $\begin{array}{l}\text { o g y } \\
\mathrm{H} 4\end{array}$ & $\begin{array}{l}\text { og y } \\
\text { H4 }\end{array}$ & o g y H12 & $\begin{array}{l}\text { o g y } \\
\text { H12 }\end{array}$ \\
\hline HEG7 & $\mathrm{HAl}$ & 51.27 & 10.41 & $\mathrm{k} 0$ y 0 & $\begin{array}{l}\text { o } 0 y \\
\mathrm{H} 4\end{array}$ & o 0 y 0 & $\begin{array}{l}\text { o i y } \\
\text { H4 }\end{array}$ & $\begin{array}{l}\text { o i y } \\
\text { H4 }\end{array}$ & o i y H4 & $\begin{array}{l}\text { o a y } \\
\mathrm{H} 4\end{array}$ & $\begin{array}{l}\text { o a y } \\
\mathrm{H} 4\end{array}$ & $\begin{array}{l}\text { o a y } \\
\text { H4 }\end{array}$ & o a y H12 & $\begin{array}{l}\text { o a y } \\
\text { H12 }\end{array}$ \\
\hline HEG8 & $\mathrm{HAl}$ & 51.27 & 10.42 & $\mathrm{k} 0$ y 0 & $\begin{array}{l}\text { o } 0 y \\
\text { H4 }\end{array}$ & o 0 y 0 & $\begin{array}{l}\text { o iy } \\
\text { H4 }\end{array}$ & $\begin{array}{l}\text { o i y } \\
\text { H4 }\end{array}$ & o i y H4 & $\begin{array}{l}\text { o a y } \\
\mathrm{H} 4\end{array}$ & $\begin{array}{l}\text { o a y } \\
\text { H4 }\end{array}$ & $\begin{array}{l}\text { o a y } \\
\text { H4 }\end{array}$ & o a y H12 & $\begin{array}{l}\text { o a y } \\
\text { H12 }\end{array}$ \\
\hline HEG9 & $\mathrm{HAl}$ & 51.22 & 10.38 & $\begin{array}{l}\text { og y } \\
\mathrm{H} 1 / \\
\mathrm{H} 18\end{array}$ & $\begin{array}{l}\text { o g y } \\
\text { H1/ } \\
\text { H18 }\end{array}$ & $\begin{array}{l}\text { og y } \\
\text { H1/ } \\
\text { H18 }\end{array}$ & $\begin{array}{l}\text { ogy } \\
\mathrm{H} 4\end{array}$ & og y 0 & $\begin{array}{l}\text { o g y } \\
\text { H4/H18 }\end{array}$ & $\begin{array}{l}\text { ogy } \\
\mathrm{H} 4\end{array}$ & $\begin{array}{l}\text { o a y } \\
\mathrm{H} 4\end{array}$ & $\begin{array}{l}\text { og y } \\
\mathrm{H} 4\end{array}$ & k 0 y H16 & $\begin{array}{l}\text { k } 0 y \\
\text { H16 }\end{array}$ \\
\hline SEG1 & $\mathrm{SCH}$ & 53.09 & 13.97 & $\mathrm{k} 0 \mathrm{n} 0$ & $\mathrm{k} 0 \mathrm{nO}$ & k 0 n 0 & $\mathrm{k} 0 \mathrm{n} 0$ & $\mathrm{k} 0 \mathrm{n} 0$ & $\mathrm{k} 0 \mathrm{n} 0$ & $\mathrm{k} 0 \mathrm{n} 0$ & $\mathrm{k} 0 \mathrm{n} 0$ & $\mathrm{k} 0 \mathrm{n} 0$ & $\mathrm{k} 0 \mathrm{n} 0$ & $\mathrm{k} 0 \mathrm{nO}$ \\
\hline SEG10 & $\mathrm{SCH}$ & 53.11 & 14 & $\mathrm{k} 0 \mathrm{n} 0$ & $\mathrm{k} 0 \mathrm{nO}$ & k 0 n 0 & $\mathrm{k} 0 \mathrm{n} 0$ & $\mathrm{k} 0 \mathrm{n} 0$ & $\mathrm{k} 0 \mathrm{n} 0$ & $\mathrm{k} 0 \mathrm{n} 0$ & $\mathrm{k} 0 \mathrm{n} 0$ & $\mathrm{k} 0 \mathrm{n} 0$ & $\mathrm{k} 0 \mathrm{n} 0$ & $\mathrm{k} 0 \mathrm{nO}$ \\
\hline SEG11 & $\mathrm{SCH}$ & 53.11 & 13.99 & $\mathrm{k} 0 \mathrm{n} 0$ & $\mathrm{k} 0 \mathrm{nO}$ & k 0 n 0 & $\mathrm{k} 0 \mathrm{n} 0$ & $\mathrm{k} 0 \mathrm{n} 0$ & $\mathrm{k} 0 \mathrm{nO}$ & $\mathrm{k} 0 \mathrm{n} 0$ & $\mathrm{k} 0 \mathrm{n} 0$ & $\mathrm{k} 0 \mathrm{n} 0$ & $\mathrm{k} 0 \mathrm{n} 0$ & $\mathrm{k} 0 \mathrm{nO}$ \\
\hline SEG12 & $\mathrm{SCH}$ & 53.09 & 13.97 & $\mathrm{k} 0 \mathrm{n} 0$ & $\mathrm{k} 0 \mathrm{nO}$ & $\mathrm{k} 0 \mathrm{n} 0$ & $\mathrm{k} 0 \mathrm{n} 0$ & $\mathrm{k} 0 \mathrm{n} 0$ & $\mathrm{k} 0 \mathrm{nO}$ & $\mathrm{k} 0 \mathrm{n} 0$ & k $0 \mathrm{n} 0$ & $\mathrm{k} 0 \mathrm{n} 0$ & $\mathrm{k} 0 \mathrm{n} 0$ & $\mathrm{k} 0 \mathrm{nO}$ \\
\hline SEG13 & $\mathrm{SCH}$ & 52.97 & 13.82 & $\begin{array}{l}\text { o a y } \\
\text { S2 }\end{array}$ & $\begin{array}{l}\text { o a y } \\
\text { S6/S2/ } \\
\text { S7 }\end{array}$ & $\begin{array}{l}\text { o a y } \\
\text { S6/S2/ } \\
\text { S7 }\end{array}$ & $\begin{array}{l}\text { o a y } \\
\text { S6/S7 }\end{array}$ & $\begin{array}{l}\text { o a y } \\
\text { S6/S7 }\end{array}$ & $\begin{array}{l}\text { o a y } \\
\text { S11/S8/ } \\
\text { S12/S9 }\end{array}$ & $\begin{array}{l}\text { o a y } \\
\text { S11/S8/ } \\
\text { S12/S9 }\end{array}$ & $\begin{array}{l}\text { o a y } \\
\text { S11/S8/ } \\
\text { s12/S9 }\end{array}$ & $\begin{array}{l}\text { o a y } \\
\text { S11/S8/ } \\
\text { s12/S9 }\end{array}$ & o a y S17 & $\begin{array}{l}\text { o a y } \\
\text { S17 }\end{array}$ \\
\hline SEG14 & $\mathrm{SCH}$ & 53.09 & 13.98 & $\mathrm{k} 0 \mathrm{nO}$ & $\mathrm{k} 0 \mathrm{nO}$ & $\mathrm{k} 0 \mathrm{n} 0$ & $\mathrm{kOn} 0$ & $\mathrm{k} 0 \mathrm{nO}$ & $\mathrm{k} 0 \mathrm{n} 0$ & $\mathrm{k} 0 \mathrm{n} 0$ & $\mathrm{k} 0 \mathrm{n} 0$ & $\begin{array}{l}\text { o c y } \\
\text { s12/S9 }\end{array}$ & $\begin{array}{l}\text { o c y S15/ } \\
\text { S17 }\end{array}$ & $\begin{array}{l}\text { oc y } \\
\text { S15/ } \\
\text { S17 }\end{array}$ \\
\hline
\end{tabular}




\begin{tabular}{|c|c|c|c|c|c|c|c|c|c|c|c|c|c|c|}
\hline EP_Plot_ID & Explo & Latitude & Longitude & 2006 & 2007 & 2008 & 2009 & 2010 & 2011 & 2012 & 2013 & 2014 & 2015 & 2016 \\
\hline SEG15 & $\mathrm{SCH}$ & 53.11 & 14.01 & $\begin{array}{l}\text { k0y } \\
\text { S3 }\end{array}$ & $\begin{array}{l}\text { k0y } \\
\text { S3 }\end{array}$ & $\begin{array}{l}\text { k } 0 \text { y } \\
\text { S3 }\end{array}$ & $\mathrm{k} 0 \mathrm{n} 0$ & $\mathrm{k} 0 \mathrm{nO}$ & k 0 n 0 & $\mathrm{k} 0 \mathrm{n} 0$ & kOn 0 & $\begin{array}{l}k 0 y \\
\text { S10 }\end{array}$ & k 0 y S15 & $\mathrm{k} 0 \mathrm{nO}$ \\
\hline SEG16 & $\mathrm{SCH}$ & 53.12 & 14 & $\begin{array}{l}\text { k } 0 y \\
\text { S3 }\end{array}$ & $\begin{array}{l}\text { k } 0 \text { y } \\
\text { S3 }\end{array}$ & $\begin{array}{l}\text { k0y } \\
\text { S3 }\end{array}$ & $\mathrm{k} 0 \mathrm{n} 0$ & $\mathrm{k} 0 \mathrm{n} 0$ & k 0 n 0 & $\mathrm{k} 0 \mathrm{n} 0$ & $\mathrm{k} 0 \mathrm{n} 0$ & $\begin{array}{l}k 0 y \\
S 10\end{array}$ & k 0 y S15 & $\mathrm{k} 0 \mathrm{n} 0$ \\
\hline SEG17 & $\mathrm{SCH}$ & 53.1 & 13.63 & $\begin{array}{l}\text { ocy } \\
\text { S14/ } \\
\text { S12 }\end{array}$ & $\begin{array}{l}\text { ocy } \\
\text { S14/ } \\
\text { S12 }\end{array}$ & $\begin{array}{l}\text { ocy } \\
\text { S14/ } \\
\text { S12 }\end{array}$ & $\begin{array}{l}\text { ocy } \\
\text { S14/ } \\
\text { S12 }\end{array}$ & $\begin{array}{l}\text { oc y } \\
\text { S14/ } \\
\text { S12 }\end{array}$ & $\begin{array}{l}\text { ocy } \\
\text { S14/ } \\
\text { S12/S9 }\end{array}$ & $\begin{array}{l}\text { ocy } \\
\text { S14/ } \\
\text { S12/S9 }\end{array}$ & $\begin{array}{l}\text { oc y } \\
\text { S14/ } \\
\text { S12/S9 }\end{array}$ & $\begin{array}{l}\text { ocy } \\
\text { S14/ } \\
\text { S12/S9 }\end{array}$ & $\begin{array}{l}\text { o c y S15/ } \\
\text { S17 }\end{array}$ & $\begin{array}{l}\text { ocy } \\
\text { S15/ } \\
\text { S17/S1 }\end{array}$ \\
\hline SEG18 & $\mathrm{SCH}$ & 53.14 & 13.88 & $\begin{array}{l}\text { o a y } \\
\text { S13/ } \\
\text { S5/S3 }\end{array}$ & o a y 0 & o a y 0 & $\begin{array}{l}\text { o a y } \\
\text { S12 }\end{array}$ & $\begin{array}{l}\text { o a y } \\
\text { S12 }\end{array}$ & $\begin{array}{l}\text { o a y } \\
\text { S12/S9 }\end{array}$ & $\begin{array}{l}\text { o a y } \\
\text { S18/ } \\
\text { S12/S9 }\end{array}$ & $\begin{array}{l}\text { o a y } \\
\text { S18/ } \\
\text { S12/S9 }\end{array}$ & $\begin{array}{l}\text { o a y } \\
\text { S18/ } \\
\text { S12/S9 }\end{array}$ & $\begin{array}{l}\text { o a y S15/ } \\
\text { S17 }\end{array}$ & $\begin{array}{l}\text { o a y } \\
\text { S15/ } \\
\text { S17 }\end{array}$ \\
\hline SEG19 & $\mathrm{SCH}$ & 53.12 & 14.01 & $\begin{array}{l}\text { k0y } \\
\text { S3 }\end{array}$ & $\begin{array}{l}\mathrm{k} 0 \mathrm{y} \\
\mathrm{S} 3\end{array}$ & $\begin{array}{l}\text { k0y } \\
\text { S3 }\end{array}$ & $\mathrm{k} 0 \mathrm{n} 0$ & $\mathrm{k} 0 \mathrm{n} 0$ & k 0 n 0 & $\mathrm{k} 0 \mathrm{n} 0$ & $\mathrm{k} 0 \mathrm{n} 0$ & k 0 n 0 & $\mathrm{k} 0 \mathrm{n} 0$ & $\mathrm{k} 0 \mathrm{n} 0$ \\
\hline SEG2 & $\mathrm{SCH}$ & 53.09 & 13.98 & $\mathrm{k} 0 \mathrm{n} 0$ & $\mathrm{k} 0 \mathrm{n} 0$ & $\mathrm{k} 0 \mathrm{n} 0$ & $\mathrm{k} 0 \mathrm{n} 0$ & $\mathrm{k} 0 \mathrm{nO}$ & k 0 n 0 & $\mathrm{k} 0 \mathrm{n} 0$ & $\mathrm{k} 0 \mathrm{n} 0$ & k 0 n 0 & $\mathrm{k} 0 \mathrm{n} 0$ & $\mathrm{k} 0 \mathrm{n} 0$ \\
\hline SEG20 & $\mathrm{SCH}$ & 53.1 & 13.62 & $\begin{array}{l}\text { ocy } \\
\text { S14/ } \\
\text { S12 }\end{array}$ & $\begin{array}{l}\text { ocy } \\
\text { S14/ } \\
\text { S12 }\end{array}$ & $\begin{array}{l}\text { oc y } \\
\text { S14/ } \\
\text { S12 }\end{array}$ & $\begin{array}{l}\text { ocy } \\
\text { S14/ } \\
\text { S12 }\end{array}$ & $\begin{array}{l}\text { oc y } \\
\text { S14/ } \\
\text { S12 }\end{array}$ & $\begin{array}{l}\text { ocy } \\
\text { S12/S9 }\end{array}$ & $\begin{array}{l}\text { o cy } \\
\text { S12/S9 }\end{array}$ & $\begin{array}{l}\text { ocy } \\
\text { S12/S9 }\end{array}$ & $\begin{array}{l}\text { ocy } \\
\text { S14/ } \\
\text { S12/S9 }\end{array}$ & $\begin{array}{l}\text { o c y S15/ } \\
\text { S17 }\end{array}$ & $\begin{array}{l}\text { ocy } \\
\text { S15/ } \\
\text { S17/S1 }\end{array}$ \\
\hline SEG21 & $\mathrm{SCH}$ & 53.11 & 13.61 & $\begin{array}{l}\text { ocy } \\
\text { S14/ } \\
\text { S12 }\end{array}$ & $\begin{array}{l}\text { oc y } \\
\text { S14/ } \\
\text { S12 }\end{array}$ & $\begin{array}{l}\text { ocy } \\
\text { S14/ } \\
\text { S12 }\end{array}$ & $\begin{array}{l}\text { ocy } \\
\text { S14/ } \\
\text { S12 }\end{array}$ & $\begin{array}{l}\text { ocy } \\
\text { S14/ } \\
\text { S12 }\end{array}$ & $\begin{array}{l}\text { ocy } \\
\text { S12/S9 }\end{array}$ & $\begin{array}{l}\text { ocy } \\
\text { S12/S9 }\end{array}$ & $\begin{array}{l}\text { ocy } \\
\text { S12/S9 }\end{array}$ & $\begin{array}{l}\text { ocy } \\
\text { S14/ } \\
\text { S12/S9 }\end{array}$ & $\begin{array}{l}\text { o c y S15/ } \\
\text { S17 }\end{array}$ & $\begin{array}{l}\text { ocy } \\
\text { S15/ } \\
\text { S17/S1 }\end{array}$ \\
\hline SEG22 & $\mathrm{SCH}$ & 53.1 & 13.97 & $\mathrm{k} 0 \mathrm{n} 0$ & kon 0 & $\mathrm{k} 0 \mathrm{n} 0$ & $\mathrm{k} 0 \mathrm{n} 0$ & $\mathrm{k} 0 \mathrm{n} 0$ & kono & $\mathrm{k} 0 \mathrm{n} 0$ & k 0 n 0 & k 0 n 0 & $\mathrm{k} 0 \mathrm{n} 0$ & k 0 n 0 \\
\hline SEG23 & $\mathrm{SCH}$ & 53.11 & 14.03 & $\begin{array}{l}\text { k } 0 y \\
\text { S3 }\end{array}$ & $\begin{array}{l}\mathrm{k} 0 \mathrm{y} \\
\mathrm{S} 3\end{array}$ & $\begin{array}{l}\text { k0y } \\
\text { S3 }\end{array}$ & $\mathrm{k} 0 \mathrm{n} 0$ & $\mathrm{k} 0 \mathrm{n} 0$ & k 0 n 0 & $\mathrm{k} 0 \mathrm{n} 0$ & $\mathrm{k} 0 \mathrm{n} 0$ & $\begin{array}{l}k 0 y \\
S 10\end{array}$ & $\mathrm{k} 0 \mathrm{n} 0$ & $\mathrm{k} 0 \mathrm{n} 0$ \\
\hline SEG24 & $\mathrm{SCH}$ & 53.09 & 14 & $\begin{array}{l}\text { k } 0 \text { y } \\
\text { S5/S3 }\end{array}$ & $\begin{array}{l}\text { k0y } \\
\text { S3 }\end{array}$ & $\begin{array}{l}\text { k0y } \\
\text { S3 }\end{array}$ & $\begin{array}{l}\text { k } 0 y \\
\text { S3 }\end{array}$ & $\begin{array}{l}\text { k } 0 \text { y } \\
\text { S3 }\end{array}$ & $\begin{array}{l}\mathrm{k} 0 \mathrm{y} \\
\mathrm{S} 10\end{array}$ & $\begin{array}{l}\mathrm{k} 0 \mathrm{y} \\
\mathrm{S} 10\end{array}$ & $\begin{array}{l}k 0 y \\
S 10\end{array}$ & $\begin{array}{l}k 0 y \\
S 10\end{array}$ & $\mathrm{k} 0 \mathrm{n} 0$ & $\mathrm{k} 0 \mathrm{n} 0$ \\
\hline SEG25 & $\mathrm{SCH}$ & 53.11 & 13.62 & $\begin{array}{l}\text { ocy } \\
\text { S14/ } \\
\text { S12 }\end{array}$ & $\begin{array}{l}\text { ocy } \\
\text { S14/ } \\
\text { S12 }\end{array}$ & $\begin{array}{l}\text { ocy } \\
\text { S14/ } \\
\text { S12 }\end{array}$ & $\begin{array}{l}\text { ocy } \\
\text { S14/ } \\
\text { S12 }\end{array}$ & $\begin{array}{l}\text { oc y } \\
\text { S14/ } \\
\text { S12 }\end{array}$ & $\begin{array}{l}\text { ocy } \\
\text { S12/S9 }\end{array}$ & $\begin{array}{l}\text { ocy } \\
\text { S12/S9 }\end{array}$ & $\begin{array}{l}\text { ocy } \\
\text { S12/S9 }\end{array}$ & $\begin{array}{l}\text { ocy } \\
\text { S14/ } \\
\text { S12/S9 }\end{array}$ & $\begin{array}{l}\text { o c y S15/ } \\
\text { S17 }\end{array}$ & $\begin{array}{l}\text { ocy } \\
\text { S15/ } \\
\text { S17/S1 }\end{array}$ \\
\hline SEG26 & $\mathrm{SCH}$ & 53.11 & 14.02 & $\begin{array}{l}\text { k } 0 y \\
\text { S3 }\end{array}$ & $\begin{array}{l}\mathrm{k} 0 \mathrm{y} \\
\mathrm{S} 3\end{array}$ & $\begin{array}{l}\text { k0y } \\
\text { S3 }\end{array}$ & $\mathrm{k} 0 \mathrm{n} 0$ & $\mathrm{k} 0 \mathrm{n} 0$ & $\mathrm{k} 0 \mathrm{n} 0$ & $\mathrm{k} 0 \mathrm{n} 0$ & $\mathrm{k} 0 \mathrm{n} 0$ & $\begin{array}{l}k 0 y \\
S 10\end{array}$ & $\mathrm{k} 0 \mathrm{n} 0$ & $\mathrm{k} 0 \mathrm{n} 0$ \\
\hline SEG27 & $\mathrm{SCH}$ & 53.12 & 13.71 & $\begin{array}{l}\text { o a y } \\
\text { S14 }\end{array}$ & $\begin{array}{l}\text { o a y } \\
\text { S12 }\end{array}$ & $\begin{array}{l}\text { o a y } \\
\text { S12 }\end{array}$ & $\begin{array}{l}\text { o a y } \\
\text { S12 }\end{array}$ & $\begin{array}{l}\text { o a y } \\
\text { S12 }\end{array}$ & $\begin{array}{l}\text { o a y } \\
\text { S12 }\end{array}$ & $\begin{array}{l}\text { o a y } \\
\text { S12 }\end{array}$ & $\begin{array}{l}\text { o a y } \\
\text { S12 }\end{array}$ & $\begin{array}{l}\text { o a y } \\
\text { S12/S9 }\end{array}$ & $\begin{array}{l}\text { o a y S15/ } \\
\text { S17 }\end{array}$ & $\begin{array}{l}\text { o a y } \\
\text { S15/ } \\
\text { S17 }\end{array}$ \\
\hline SEG28 & $\mathrm{SCH}$ & 53.09 & 14.01 & $\begin{array}{l}\text { k0y } \\
\text { S3 }\end{array}$ & $\begin{array}{l}\text { k } 0 y \\
\text { S3 }\end{array}$ & $\begin{array}{l}\text { k0y } \\
\text { s3 }\end{array}$ & $\begin{array}{l}\text { k } 0 \text { y } \\
\text { S3 }\end{array}$ & $\begin{array}{l}\text { k } 0 y \\
\text { S3 }\end{array}$ & $\begin{array}{l}k 0 y \\
\text { S10 }\end{array}$ & $\begin{array}{l}\mathrm{k} 0 \mathrm{y} \\
\mathrm{S} 10\end{array}$ & $\begin{array}{l}k 0 y \\
S 10\end{array}$ & $\begin{array}{l}k 0 y \\
\text { S10 }\end{array}$ & $\mathrm{k} 0 \mathrm{n} 0$ & $\mathrm{k} 0 \mathrm{n} 0$ \\
\hline SEG29 & $\mathrm{SCH}$ & 53.09 & 14 & $\begin{array}{l}\text { k0y } \\
\text { S3 }\end{array}$ & $\begin{array}{l}\text { k0y } \\
\text { S3 }\end{array}$ & $\begin{array}{l}\text { k0y } \\
\text { S3 }\end{array}$ & $\begin{array}{l}\mathrm{k} 0 \mathrm{y} \\
\mathrm{S} 3\end{array}$ & $\begin{array}{l}k 0 y \\
\text { s3 }\end{array}$ & $\begin{array}{l}k 0 y \\
\text { S10 }\end{array}$ & $\begin{array}{l}\text { k } 0 y \\
\text { S10 }\end{array}$ & $\begin{array}{l}k 0 y \\
S 10\end{array}$ & $\begin{array}{l}k 0 y \\
\text { S10 }\end{array}$ & $\mathrm{k} 0 \mathrm{n} 0$ & $\mathrm{k} 0 \mathrm{n} 0$ \\
\hline
\end{tabular}




\begin{tabular}{|c|c|c|c|c|c|c|c|c|c|c|c|c|c|c|}
\hline EP_Plot_ID & Explo & Latitude & Longitude & 2006 & 2007 & 2008 & 2009 & 2010 & 2011 & 2012 & 2013 & 2014 & 2015 & 2016 \\
\hline SEG3 & $\mathrm{SCH}$ & 53.1 & 13.99 & $\mathrm{k} 0 \mathrm{n} 0$ & $\mathrm{k} 0 \mathrm{n} 0$ & $\mathrm{k} 0 \mathrm{n} 0$ & $\mathrm{k} 0 \mathrm{n} 0$ & $\mathrm{k} 0 \mathrm{n} 0$ & k 0 n 0 & $\mathrm{k} 0 \mathrm{n} 0$ & $\mathrm{k} 0 \mathrm{n} 0$ & $\mathrm{k} 0 \mathrm{n} 0$ & $\mathrm{k} 0 \mathrm{nO}$ & $\mathrm{k} 0 \mathrm{nO}$ \\
\hline SEG30 & $\mathrm{SCH}$ & 53.15 & 13.83 & $\begin{array}{l}\text { o a y } \\
\text { S4/S3/ } \\
\text { S14 }\end{array}$ & $\begin{array}{l}\text { o a y } \\
\text { S3/ } \\
\text { S14 }\end{array}$ & $\begin{array}{l}\text { o a y } \\
\text { S12 }\end{array}$ & $\begin{array}{l}\text { o a y } \\
\text { S12 }\end{array}$ & $\begin{array}{l}\text { o a y } \\
\text { S12 }\end{array}$ & $\begin{array}{l}\text { o a y } \\
\text { S12 }\end{array}$ & $\begin{array}{l}\text { o a y } \\
\text { S12 }\end{array}$ & $\begin{array}{l}\text { o a y } \\
\text { S12 }\end{array}$ & $\begin{array}{l}\text { o a y } \\
\text { S12 }\end{array}$ & $\begin{array}{l}\text { o a y S15/ } \\
\text { S17 }\end{array}$ & $\begin{array}{l}\text { o a y } \\
\text { S15/ } \\
\text { S17 }\end{array}$ \\
\hline SEG31 & $\mathrm{SCH}$ & 53.15 & 13.84 & $\begin{array}{l}\text { o a y } \\
\text { S4/S3/ } \\
\text { S14 }\end{array}$ & $\begin{array}{l}\text { o a y } \\
\text { S3/ } \\
\text { S14 }\end{array}$ & $\begin{array}{l}\text { o a y } \\
\text { S12 }\end{array}$ & $\begin{array}{l}\text { o a y } \\
\text { S12 }\end{array}$ & $\begin{array}{l}\text { o a y } \\
\text { S12 }\end{array}$ & $\begin{array}{l}\text { o a y } \\
\text { S12 }\end{array}$ & $\begin{array}{l}\text { o a y } \\
\text { S12 }\end{array}$ & $\begin{array}{l}\text { o a y } \\
\text { S12 }\end{array}$ & $\begin{array}{l}\text { o a y } \\
\text { S12 }\end{array}$ & $\begin{array}{l}\text { o a y S15/ } \\
\text { S17 }\end{array}$ & $\begin{array}{l}\text { o a y } \\
\text { S15/ } \\
\text { S17 }\end{array}$ \\
\hline SEG32 & $\mathrm{SCH}$ & 53.15 & 13.83 & $\begin{array}{l}\text { o a y } \\
\text { S4/S3/ } \\
\text { S14 }\end{array}$ & $\begin{array}{l}\text { o a y } \\
\text { S3/ } \\
\text { S14 }\end{array}$ & $\begin{array}{l}\text { o a y } \\
\text { S12 }\end{array}$ & $\begin{array}{l}\text { o a y } \\
\text { s12 }\end{array}$ & $\begin{array}{l}\text { o a y } \\
\text { S12 }\end{array}$ & $\begin{array}{l}\text { o a y } \\
\text { S12 }\end{array}$ & $\begin{array}{l}\text { o a y } \\
\text { S12 }\end{array}$ & $\begin{array}{l}\text { o a y } \\
\text { S12 }\end{array}$ & $\begin{array}{l}\text { o a y } \\
\text { S12 }\end{array}$ & $\begin{array}{l}\text { o a y S15/ } \\
\text { S17 }\end{array}$ & $\begin{array}{l}\text { o a y } \\
\text { S15/ } \\
\text { S17 }\end{array}$ \\
\hline SEG33 & $\mathrm{SCH}$ & 52.99 & 13.84 & $\begin{array}{l}\text { o a y } \\
\text { S2 }\end{array}$ & $\begin{array}{l}\text { o a y } \\
\text { S2/S7 }\end{array}$ & $\begin{array}{l}\text { o a y } \\
\text { S2/S7 }\end{array}$ & $\begin{array}{l}\text { o a y } \\
\text { S7 }\end{array}$ & $\begin{array}{l}\text { o a y } \\
\text { S7 }\end{array}$ & $\begin{array}{l}\text { o a y } \\
\text { S12/S9 }\end{array}$ & $\begin{array}{l}\text { o a y } \\
\text { S12/S9 }\end{array}$ & $\begin{array}{l}\text { o a y } \\
\text { S12/S9 }\end{array}$ & $\begin{array}{l}\text { o a y } \\
\text { S12/S9 }\end{array}$ & $\begin{array}{l}\text { o a y S15/ } \\
\text { S17 }\end{array}$ & $\begin{array}{l}\text { o a y } \\
\text { S15/ } \\
\text { S17 }\end{array}$ \\
\hline SEG34 & $\mathrm{SCH}$ & 52.98 & 13.85 & $\begin{array}{l}\text { o a y } \\
\text { S2 }\end{array}$ & $\begin{array}{l}\text { o a y } \\
\text { S2/S7 }\end{array}$ & $\begin{array}{l}\text { o a y } \\
\text { S2/S7 }\end{array}$ & $\begin{array}{l}\text { o a y } \\
\text { S7 }\end{array}$ & $\begin{array}{l}\text { o a y } \\
\text { S7 }\end{array}$ & $\begin{array}{l}\text { o a y } \\
\text { S12/S9 }\end{array}$ & $\begin{array}{l}\text { o a y } \\
\text { S12/S9 }\end{array}$ & $\begin{array}{l}\text { o a y } \\
\text { S12/S9 }\end{array}$ & $\begin{array}{l}\text { o a y } \\
\text { S12/S9 }\end{array}$ & $\begin{array}{l}\text { o a y S15/ } \\
\text { S17 }\end{array}$ & $\begin{array}{l}\text { o a y } \\
\text { S15/ } \\
\text { S17 }\end{array}$ \\
\hline SEG35 & $\mathrm{SCH}$ & 52.98 & 13.85 & $\begin{array}{l}\text { o a y } \\
\text { S2 }\end{array}$ & $\begin{array}{l}\text { o a y } \\
\text { S2/S7 }\end{array}$ & $\begin{array}{l}\text { o a y } \\
\text { S2/S7 }\end{array}$ & $\begin{array}{l}\text { o a y } \\
\text { S7 }\end{array}$ & $\begin{array}{l}\text { o a y } \\
\text { S7 }\end{array}$ & $\begin{array}{l}\text { o a y } \\
\text { S12/S9 }\end{array}$ & $\begin{array}{l}\text { o a y } \\
\text { S12/S9 }\end{array}$ & $\begin{array}{l}\text { o a y } \\
\text { S12/S9 }\end{array}$ & $\begin{array}{l}\text { o a y } \\
\text { S12/S9 }\end{array}$ & $\begin{array}{l}\text { o a y S15/ } \\
\text { S17 }\end{array}$ & $\begin{array}{l}\text { o a y } \\
\text { S15/ } \\
\text { S17 }\end{array}$ \\
\hline SEG36 & $\mathrm{SCH}$ & 52.99 & 13.84 & $\begin{array}{l}\text { o a y } \\
\text { S2 }\end{array}$ & $\begin{array}{l}\text { o a y } \\
\text { S2/S7 }\end{array}$ & $\begin{array}{l}\text { o a y } \\
\text { S2/S7 }\end{array}$ & $\begin{array}{l}\text { o a y } \\
\text { S7 }\end{array}$ & $\begin{array}{l}\text { o a y } \\
\text { S7 }\end{array}$ & $\begin{array}{l}\text { o a y } \\
\text { S12/S9 }\end{array}$ & $\begin{array}{l}\text { o a y } \\
\text { S12/S9 }\end{array}$ & $\begin{array}{l}\text { o a y } \\
\text { S12/S9 }\end{array}$ & $\begin{array}{l}\text { o a y } \\
\text { S12/S9 }\end{array}$ & $\begin{array}{l}\text { o a y S15/ } \\
\text { S17 }\end{array}$ & $\begin{array}{l}\text { o a y } \\
\text { S15/ } \\
\text { S17 }\end{array}$ \\
\hline SEG37 & $\mathrm{SCH}$ & 53.13 & 13.88 & o a y 0 & o a y 0 & o a y 0 & $\begin{array}{l}\text { o a y } \\
\text { S13 }\end{array}$ & $\begin{array}{l}\text { o a y } \\
\text { S12 }\end{array}$ & $\begin{array}{l}\text { o a y } \\
\text { S12 }\end{array}$ & $\begin{array}{l}\text { oay } \\
\text { S12 }\end{array}$ & $\begin{array}{l}\text { o a y } \\
\text { S12 }\end{array}$ & $\begin{array}{l}\text { o a y } \\
\text { S12 }\end{array}$ & $\begin{array}{l}\text { o a y S15/ } \\
\text { S17 }\end{array}$ & $\begin{array}{l}\text { o a y } \\
\text { S15/ } \\
\text { S17 }\end{array}$ \\
\hline SEG38 & $\mathrm{SCH}$ & 53.12 & 13.68 & $\begin{array}{l}\text { o a y } \\
\text { S14 }\end{array}$ & $\begin{array}{l}\text { o a y } \\
\text { S12 }\end{array}$ & $\begin{array}{l}\text { o a y } \\
\text { S12 }\end{array}$ & $\begin{array}{l}\text { o a y } \\
\text { S12 }\end{array}$ & $\begin{array}{l}\text { o a y } \\
\text { S12 }\end{array}$ & $\begin{array}{l}\text { o a y } \\
\text { S12 }\end{array}$ & $\begin{array}{l}\text { o a y } \\
\text { S12/S9 }\end{array}$ & $\begin{array}{l}\text { o a y } \\
\text { S12 }\end{array}$ & $\begin{array}{l}\text { o a y } \\
\text { S12/S9 }\end{array}$ & $\begin{array}{l}\text { o a y S15/ } \\
\text { S17 }\end{array}$ & $\begin{array}{l}\text { o a y } \\
\text { S15/ } \\
\text { S17 }\end{array}$ \\
\hline SEG39 & $\mathrm{SCH}$ & 52.98 & 13.82 & $\begin{array}{l}\text { o a y } \\
\text { S2 }\end{array}$ & $\begin{array}{l}\text { o a y } \\
\text { S2/S7 }\end{array}$ & $\begin{array}{l}\text { o a y } \\
\text { S2/S7 }\end{array}$ & $\begin{array}{l}\text { o a y } \\
\text { S7 }\end{array}$ & $\begin{array}{l}\text { o a y } \\
\text { S7 }\end{array}$ & $\begin{array}{l}\text { o a y } \\
\text { S12/S9 }\end{array}$ & $\begin{array}{l}\text { o a y } \\
\text { S12/S9 }\end{array}$ & $\begin{array}{l}\text { o a y } \\
\text { S12/S9 }\end{array}$ & $\begin{array}{l}\text { o a y } \\
\text { S12/S9 }\end{array}$ & $\begin{array}{l}\text { o a y S15/ } \\
\text { S17 }\end{array}$ & $\begin{array}{l}\text { o a y } \\
\text { S15/ } \\
\text { S17 }\end{array}$ \\
\hline SEG4 & $\mathrm{SCH}$ & 53.11 & 14 & $\begin{array}{l}\text { k0y } \\
\text { S3 }\end{array}$ & $\begin{array}{l}\text { k0y } \\
\text { S3 }\end{array}$ & $\begin{array}{l}\mathrm{k} 0 \mathrm{y} \\
\mathrm{s} 3\end{array}$ & $\mathrm{k} 0 \mathrm{n} 0$ & k 0 n 0 & $\mathrm{k} 0 \mathrm{n} 0$ & $\mathrm{k} 0 \mathrm{n} 0$ & $\mathrm{k} 0 \mathrm{n} 0$ & $\begin{array}{l}\mathrm{k} 0 \mathrm{y} \\
\mathrm{S} 10\end{array}$ & k 0 y S15 & $\mathrm{k} 0 \mathrm{no}$ \\
\hline SEG40 & $\mathrm{SCH}$ & 53.12 & 13.84 & o a y 0 & o a y 0 & o a y 0 & $\begin{array}{l}\text { o a y } \\
\text { S12 }\end{array}$ & $\begin{array}{l}\text { o a y } \\
\text { S12 }\end{array}$ & $\begin{array}{l}\text { o a y } \\
\text { S12 }\end{array}$ & $\begin{array}{l}\text { o a y } \\
\text { S12 }\end{array}$ & $\begin{array}{l}\text { o a y } \\
\text { S12 }\end{array}$ & $\begin{array}{l}\text { o a y } \\
\text { S12 }\end{array}$ & o a y $\mathrm{S} 17$ & $\begin{array}{l}\text { o a y } \\
\text { S17 }\end{array}$ \\
\hline SEG41 & $\mathrm{SCH}$ & 53.12 & 13.85 & o a y 0 & o a y 0 & o a y 0 & $\begin{array}{l}\text { o a y } \\
\text { S12 }\end{array}$ & $\begin{array}{l}\text { o a y } \\
\text { S12 }\end{array}$ & $\begin{array}{l}\text { o a y } \\
\text { S12 }\end{array}$ & $\begin{array}{l}\text { o a y } \\
\text { S12 }\end{array}$ & $\begin{array}{l}\text { o a y } \\
\text { S12 }\end{array}$ & $\begin{array}{l}\text { o a y } \\
\text { S12 }\end{array}$ & o a y $S 17$ & $\begin{array}{l}\text { o a y } \\
\text { S17 }\end{array}$ \\
\hline
\end{tabular}




\begin{tabular}{|c|c|c|c|c|c|c|c|c|c|c|c|c|c|c|}
\hline EP_Plot_ID & Explo & Latitude & Longitude & 2006 & 2007 & 2008 & 2009 & 2010 & 2011 & 2012 & 2013 & 2014 & 2015 & 2016 \\
\hline SEG42 & $\mathrm{SCH}$ & 52.87 & 13.97 & $\begin{array}{l}\text { oh y } \\
\text { S2 }\end{array}$ & $\begin{array}{l}\text { o h y } \\
\text { S2/ } \\
\text { S12 }\end{array}$ & $\begin{array}{l}\text { o hy } \\
\text { S2/ } \\
\text { S12 }\end{array}$ & $\begin{array}{l}\text { o hy } \\
\text { S7 }\end{array}$ & $\begin{array}{l}\text { o hy } \\
\text { S2/ } \\
\text { S12 }\end{array}$ & $\begin{array}{l}\text { o hy } \\
\text { S2/S12 }\end{array}$ & $\begin{array}{l}\text { o hy } \\
\text { S2/S12 }\end{array}$ & $\begin{array}{l}\text { o hy } \\
\text { S2/S12 }\end{array}$ & $\begin{array}{l}\text { o hy } \\
\text { S2/S12 }\end{array}$ & $\begin{array}{l}\text { o h y S15/ } \\
\text { S17 }\end{array}$ & $\begin{array}{l}\text { o hy } \\
\text { S15/ } \\
\text { S17 }\end{array}$ \\
\hline SEG43 & $\mathrm{SCH}$ & 52.88 & 13.97 & $\begin{array}{l}\text { o h y } \\
\text { S2 }\end{array}$ & $\begin{array}{l}\text { o h y } \\
\text { s2/ } \\
\text { S12 }\end{array}$ & $\begin{array}{l}\text { o hy } \\
\text { S2/ } \\
\text { S12 }\end{array}$ & $\begin{array}{l}\text { ohy } \\
\text { S7 }\end{array}$ & $\begin{array}{l}\text { o hy } \\
\text { S2/ } \\
\text { S12 }\end{array}$ & $\begin{array}{l}\text { o hy } \\
\text { S2/S12 }\end{array}$ & $\begin{array}{l}\text { o hy } \\
\text { S2/S12 }\end{array}$ & $\begin{array}{l}\text { o hy } \\
\text { S2/S12 }\end{array}$ & $\begin{array}{l}\text { o hy } \\
\text { S2/S12 }\end{array}$ & $\begin{array}{l}\text { o h y S15/ } \\
\text { S17 }\end{array}$ & $\begin{array}{l}\text { o hy } \\
\text { S15/ } \\
\text { S17 }\end{array}$ \\
\hline SEG44 & $\mathrm{SCH}$ & 52.88 & 13.97 & $\begin{array}{l}\text { ohy } \\
\text { S2 }\end{array}$ & $\begin{array}{l}\text { o h y } \\
\text { S2/ } \\
\text { S12 }\end{array}$ & $\begin{array}{l}\text { oh y } \\
\text { S2/ } \\
\text { S12 }\end{array}$ & $\begin{array}{l}\text { oh y } \\
\text { S7 }\end{array}$ & $\begin{array}{l}\text { o hy } \\
\text { S2/ } \\
\text { S12 }\end{array}$ & $\begin{array}{l}\text { o hy } \\
\text { S2/S12 }\end{array}$ & $\begin{array}{l}\text { o hy } \\
\text { S2/S12 }\end{array}$ & $\begin{array}{l}\text { o hy } \\
\text { S2/S12 }\end{array}$ & $\begin{array}{l}\text { o hy } \\
\text { S2/S12 }\end{array}$ & $\begin{array}{l}\text { o h y S15/ } \\
\text { S17 }\end{array}$ & $\begin{array}{l}\text { o hy } \\
\text { S15/ } \\
\text { S17 }\end{array}$ \\
\hline SEG45 & $\mathrm{SCH}$ & 52.88 & 13.96 & $\begin{array}{l}\text { oh y } \\
\text { s2 }\end{array}$ & $\begin{array}{l}\text { oh y } \\
\text { S2/ } \\
\text { S12 }\end{array}$ & $\begin{array}{l}\text { o hy } \\
\text { S2/ } \\
\text { S12 }\end{array}$ & $\begin{array}{l}\text { o h y } \\
\text { S7 }\end{array}$ & $\begin{array}{l}\text { o hy } \\
\text { S2/ } \\
\text { S12 }\end{array}$ & $\begin{array}{l}\text { o hy } \\
\text { S2/S12 }\end{array}$ & $\begin{array}{l}\text { o hy } \\
\text { s2/S12 }\end{array}$ & $\begin{array}{l}\text { ohy } \\
\text { S2/S12 }\end{array}$ & $\begin{array}{l}\text { o h y } \\
\text { S2/S12 }\end{array}$ & $\begin{array}{l}\text { o h y S15/ } \\
\text { S17 }\end{array}$ & $\begin{array}{l}\text { o hy } \\
\text { S15/ } \\
\text { S17 }\end{array}$ \\
\hline SEG46 & $\mathrm{SCH}$ & 52.98 & 13.83 & $\begin{array}{l}\text { o a y } \\
\text { S2 }\end{array}$ & $\begin{array}{l}\text { o a y } \\
\text { S2/S7 }\end{array}$ & $\begin{array}{l}\text { o a y } \\
\text { S2/S7 }\end{array}$ & $\begin{array}{l}\text { o a y } \\
\text { S7 }\end{array}$ & $\begin{array}{l}\text { o a y } \\
\text { S7 }\end{array}$ & $\begin{array}{l}\text { o a y } \\
\text { s12/S9 }\end{array}$ & $\begin{array}{l}\text { o a y } \\
\text { s12/S9 }\end{array}$ & $\begin{array}{l}\text { o a y } \\
\text { s12/S9 }\end{array}$ & $\begin{array}{l}\text { o a y } \\
\text { s12/S9 }\end{array}$ & $\begin{array}{l}\text { o a y S15/ } \\
\text { S17 }\end{array}$ & $\begin{array}{l}\text { o a y } \\
\text { S15/ } \\
\text { S17 }\end{array}$ \\
\hline SEG47 & $\mathrm{SCH}$ & 52.99 & 13.83 & $\begin{array}{l}\text { o a y } \\
\text { s2 }\end{array}$ & $\begin{array}{l}\text { o a y } \\
\text { S2/S7 }\end{array}$ & $\begin{array}{l}\text { o a y } \\
\text { S2/S7 }\end{array}$ & $\begin{array}{l}\text { o a y } \\
\text { S7 }\end{array}$ & $\begin{array}{l}\text { o a y } \\
\text { S7 }\end{array}$ & $\begin{array}{l}\text { o a y } \\
\text { S12/S9 }\end{array}$ & $\begin{array}{l}\text { o a y } \\
\text { S12/S9 }\end{array}$ & $\begin{array}{l}\text { o a y } \\
\text { S12/S9 }\end{array}$ & $\begin{array}{l}\text { o a y } \\
\text { S12/S9 }\end{array}$ & $\begin{array}{l}\text { o a y S15/ } \\
\text { S17 }\end{array}$ & $\begin{array}{l}\text { o a y } \\
\text { S15/ } \\
\text { S17 }\end{array}$ \\
\hline SEG48 & $\mathrm{SCH}$ & 53.1 & 13.61 & $\begin{array}{l}\text { ocy } \\
\text { S14/ } \\
\text { s12 }\end{array}$ & $\begin{array}{l}\text { o c y } \\
\text { S14/ } \\
\text { S12 }\end{array}$ & $\begin{array}{l}\text { o c y } \\
\text { s14/ } \\
\text { s12 }\end{array}$ & $\begin{array}{l}\text { o c y } \\
\text { S14/ } \\
\text { S12 }\end{array}$ & $\begin{array}{l}\text { o c y } \\
\text { S14/ } \\
\text { S12 }\end{array}$ & $\begin{array}{l}\text { o c y } \\
\text { S12/S9 }\end{array}$ & $\begin{array}{l}\text { o cy } \\
\text { s12/S9 }\end{array}$ & $\begin{array}{l}\text { o c y } \\
\text { s12/S9 }\end{array}$ & $\begin{array}{l}\text { o c y } \\
\text { s14/ } \\
\text { s12/S9 }\end{array}$ & $\begin{array}{l}\text { o c y S15/ } \\
\text { S17 }\end{array}$ & $\begin{array}{l}\text { o c y } \\
\text { S15/ } \\
\text { S17/S1 }\end{array}$ \\
\hline SEG49 & $\mathrm{SCH}$ & 52.97 & 13.86 & $\begin{array}{l}\text { o a y } \\
\text { s2 }\end{array}$ & $\begin{array}{l}\text { o a y } \\
\text { S2/S7 }\end{array}$ & $\begin{array}{l}\text { o a y } \\
\text { S2/S7 }\end{array}$ & $\begin{array}{l}\text { o a y } \\
\text { S7 }\end{array}$ & $\begin{array}{l}\text { o a y } \\
\text { S7 }\end{array}$ & $\begin{array}{l}\text { o a y } \\
\text { s12/S9 }\end{array}$ & $\begin{array}{l}\text { o a y } \\
\text { S12/S9 }\end{array}$ & $\begin{array}{l}\text { o a y } \\
\text { s12/S9 }\end{array}$ & $\begin{array}{l}\text { o a y } \\
\text { S12/S9 }\end{array}$ & $\begin{array}{l}\text { o a y S15/ } \\
\text { S17 }\end{array}$ & $\begin{array}{l}\text { o a y } \\
\text { S15/ } \\
\text { S17 }\end{array}$ \\
\hline SEG5 & $\mathrm{SCH}$ & 53.11 & 14 & $\begin{array}{l}\text { k } 0 \text { y } \\
\text { S3 }\end{array}$ & $\begin{array}{l}\text { k } 0 y \\
\text { s3 }\end{array}$ & $\begin{array}{l}\text { k } 0 y \\
\text { S3 }\end{array}$ & $\mathrm{k} 0 \mathrm{n} 0$ & $\mathrm{k} 0 \mathrm{n} 0$ & $\mathrm{k} 0 \mathrm{n} 0$ & $\mathrm{k} 0 \mathrm{n} 0$ & k 0 n 0 & $\begin{array}{l}\text { k } 0 \text { y } \\
\text { S10 }\end{array}$ & k 0 y S15 & k $0 \cap 0$ \\
\hline SEG50 & $\mathrm{SCH}$ & 53.12 & 13.75 & $\begin{array}{l}\text { ocy } \\
\text { S14 }\end{array}$ & $\begin{array}{l}\text { o c y } \\
\text { S14 }\end{array}$ & $\begin{array}{l}\text { o c y } \\
\text { S14 }\end{array}$ & $\begin{array}{l}\text { ocy } \\
\text { S14 }\end{array}$ & $\begin{array}{l}\text { ocy } \\
\text { S14 }\end{array}$ & $\begin{array}{l}\text { ocy } \\
\text { S14 }\end{array}$ & $\begin{array}{l}\text { ocy } \\
\text { S14 }\end{array}$ & $\begin{array}{l}\text { ocy } \\
\text { S14 }\end{array}$ & $\begin{array}{l}\text { o c y } \\
\text { S14 }\end{array}$ & $\begin{array}{l}\text { o c y S15/ } \\
\text { S17 }\end{array}$ & $\begin{array}{l}\text { o cy } \\
\text { S15/ } \\
\text { S17 }\end{array}$ \\
\hline SEG6 & $\mathrm{SCH}$ & 53.1 & 13.62 & $\begin{array}{l}\text { o c y } \\
\text { s14/ } \\
\text { s12 }\end{array}$ & $\begin{array}{l}\text { o c y } \\
\text { s14/ } \\
\text { s12 }\end{array}$ & $\begin{array}{l}\text { o c y } \\
\text { s14/ } \\
\text { s12 }\end{array}$ & $\begin{array}{l}\text { o c y } \\
\text { S14/ } \\
\text { s12 }\end{array}$ & $\begin{array}{l}\text { o c y } \\
\text { s14/ } \\
\text { s12 }\end{array}$ & $\begin{array}{l}\text { o c y } \\
\text { s14/ } \\
\text { s12/s9 }\end{array}$ & $\begin{array}{l}\text { o c y } \\
\text { s14/ } \\
\text { s12/s9 }\end{array}$ & $\begin{array}{l}\text { o c y } \\
\text { s14/ } \\
\text { s12/S9 }\end{array}$ & $\begin{array}{l}\text { o c y } \\
\text { s14/ } \\
\text { s12/S9 }\end{array}$ & $\begin{array}{l}\text { o c y S15/ } \\
\text { S17 }\end{array}$ & $\begin{array}{l}\text { o c y } \\
\text { S15/ } \\
\text { S17/S1 }\end{array}$ \\
\hline SEG7 & $\mathrm{SCH}$ & 53.09 & 13.98 & $\mathrm{k} 0 \mathrm{n} 0$ & $\mathrm{k} 0 \mathrm{n} 0$ & k 0 n 0 & $\mathrm{k} 0 \mathrm{nO}$ & $\mathrm{k} 0 \mathrm{n} 0$ & k 0 n 0 & k 0 n 0 & k 0 n 0 & k 0 n 0 & $\mathrm{k} 0 \mathrm{n} 0$ & k 0 n 0 \\
\hline SEG8 & $\mathrm{SCH}$ & 53.11 & 14.02 & $\begin{array}{l}\text { k } 0 \text { y } \\
\text { s3 }\end{array}$ & $\begin{array}{l}\text { k } 0 \text { y } \\
\text { s3 }\end{array}$ & $\begin{array}{l}\text { k } 0 y \\
\text { s3 }\end{array}$ & $\mathrm{k} 0 \mathrm{n} 0$ & k 0 n 0 & k 0 n 0 & k 0 n 0 & k 0 n 0 & $\begin{array}{l}\text { k 0 y } \\
\text { S10 }\end{array}$ & $\mathrm{k} 0$ y 0 & k 0 n 0 \\
\hline
\end{tabular}




\begin{tabular}{|l|l|l|l|l|l|l|l|l|l|l|l|l|l|l|l|}
\hline EP_Plot_ID & Explo & Latitude & Longitude & 2006 & 2007 & 2008 & 2009 & 2010 & 2011 & 2012 & 2013 & 2014 & 2015 & 2016 \\
\hline SEG9 & SCH & 53.1 & 13.61 & ocy & oc $y$ & ocy & ocy & ocy & ocy & ocy & ocy & ocy & ocy S15/ & ocy \\
& & & & $S 14 /$ & $S 14 /$ & $S 14 /$ & $S 14 /$ & $S 14 /$ & $S 14 /$ & $S 14 /$ & $S 14 /$ & $S 14 /$ & $S 17$ & $S 15 /$ \\
& & & & $S 12$ & $S 12$ & $S 12$ & $S 12$ & $S 12$ & $S 12 / S 9$ & $S 12 / S 9$ & $S 12 / S 9$ & $S 12 / S 9$ & & $S 17 / S 1$ \\
\hline
\end{tabular}


Table 6.

Description of the agri-environmental measures coding of Table 5.

$0=$ no description or not applicable (0)

Type of farming (1. digit)

$\mathrm{k}=$ conventional

$\mathrm{o}=$ ecological

Ecological directive (2. digit)

$a=$ EU-Bio

$b=$ DE-Bio

$c=$ Bioland

$d=$ Naturland

e $=$ Biokreis

$f=$ Naturland

$g$ = Demeter

$\mathrm{h}=$ Biopark

$i=$ GÄA e.V.

Agri-environmental measure (AEM) (3. digit)

$\mathrm{n}=\mathrm{no}$

$y=$ yes

Description AEM (4.digit)

Alb:

MEKA (1992-2013/2014)

$\mathrm{A} 1=\mathrm{N}-\mathrm{B}$ 1: extensive

A2 $=N-B$ 2: extensive and low livestock density

A3 $=$ N-B 3: steep slopes (inclination $\geq 25 \%$ ) - difficult conditions

A4 = N-B 4: biodiverse

A5 $=$ N-D 2: organic farming

$A 6=N-G$ 1.1: extensive management of protected biotopes

FAKT (2014-2020)

$\mathrm{A} 7=\mathrm{B}$ 1.1: extensive, $\max 1.4 \mathrm{RLU} / \mathrm{ha}$, no mineral nitrogen

$\mathrm{A} 8=\mathrm{B}$ 1.2: extensive, $\min 0.3 \mathrm{RLU} / \mathrm{ha}$, no nitrogen 


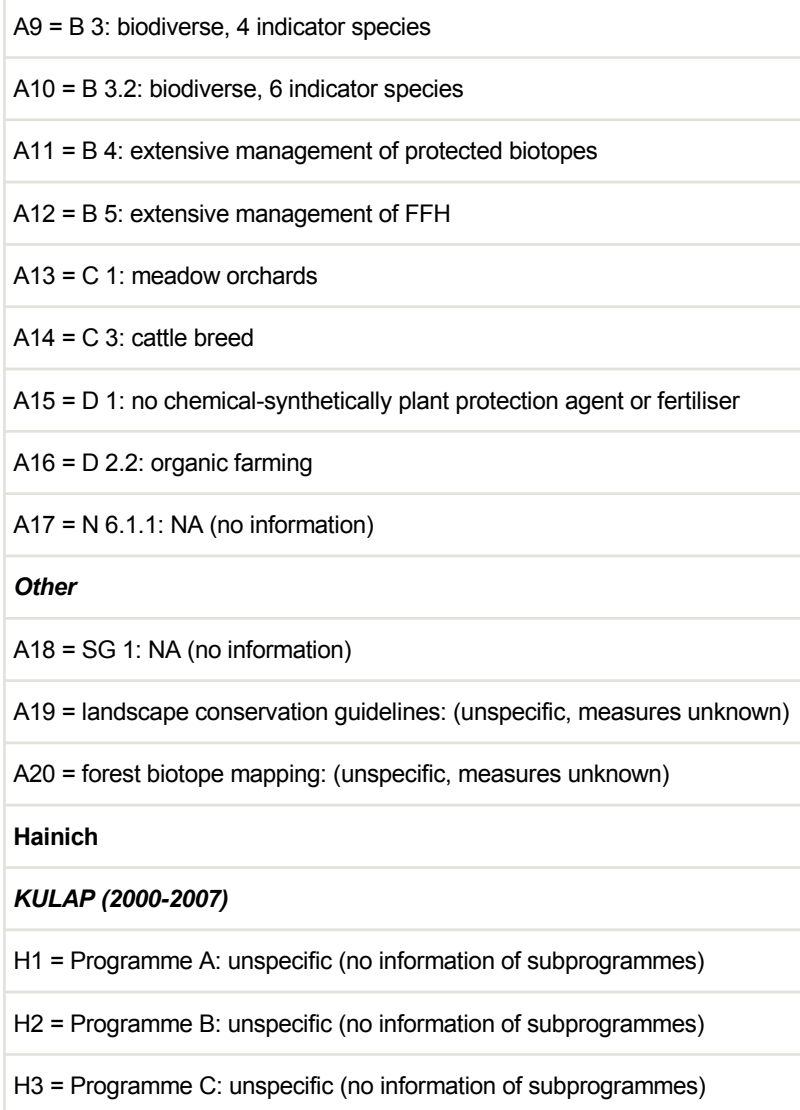




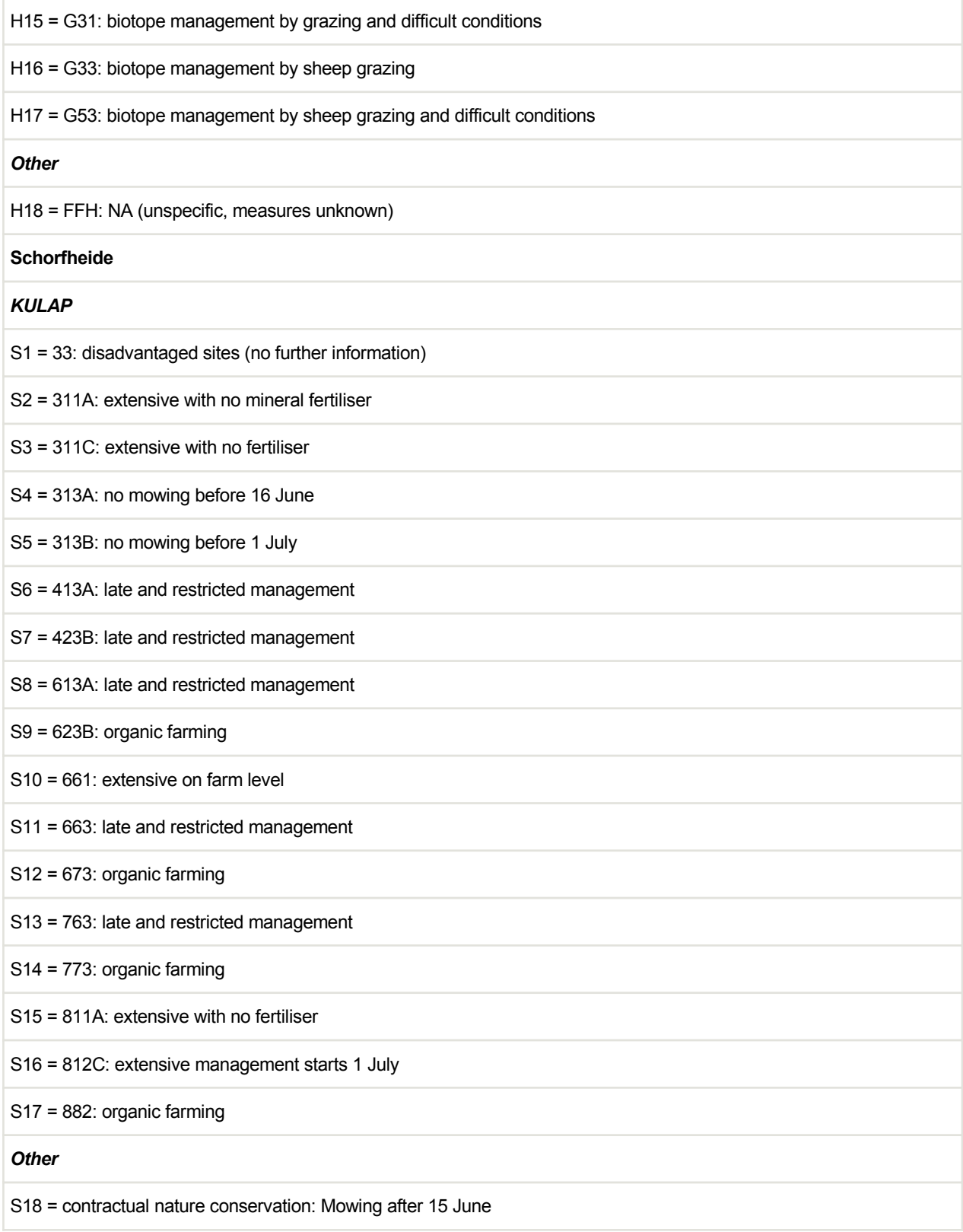

In accordance with Blüthgen et al. 2012, mowing and fertilisation were correlated such that grasslands that were mown more frequently also received higher amounts of fertiliser. High grazing intensity was correlated with low mowing frequency such that intensively grazed grasslands were not mown and vice versa (Fig. 3a). Nevertheless, many grasslands were mown pastures, i.e. they are both grazed by varying types of livestock and mown using different practices. Timing of mowing and grazing was also variable between years. Correlations between mowing and grazing and between mowing and fertilisation, were 
stronger than those between fertilisation and grazing (Fig. 3). Correlations also differed between regions (Fig. 3). For example, in Hainich, there were weaker negative correlations between mowing and grazing compared to the Schwäbische Alb and Schorfheide.

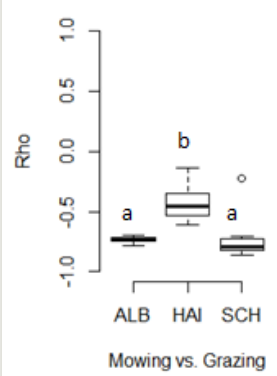

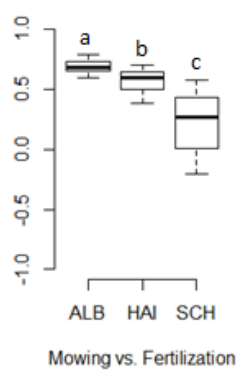

b)

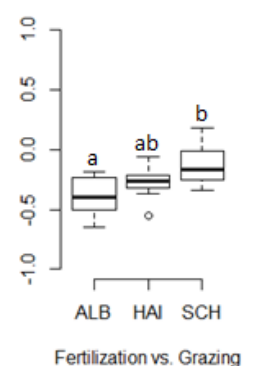

c)

Figure 3. doi

Boxplots showing the Spearman correlation coefficients (Rho) between the different grassland management components, calculated separately for each 11 years. a) mowing vs. grazing, b) mowing vs. fertilisation and c) fertilisation vs. grazing of the three different regions (ALB- Swabian Alb, HAl- Hainich, SCH- Schorfheide). Boxes with the same letters are not significantly different at $p>0.05$ using pairwise Wilcoxon tests with Bonferroni correction.

The value of this dataset lies in the comprehensive and consistent description and characterisation of grassland management of 150 grassland plots over 11 years. Detailed accounting of land-use practices can only be achieved through intensive collaboration between land managers, land users and researchers, as done in our study. The accuracy of the answers given by farmers strongly determined the data quality. While in our study regions, all farmers had to keep records of management, mainly due to regulations of EU agricultural subsidies and cross-compliance obligations, the quality of the records still differed in some detail. To increase the accuracy of the data, members of the BE project additionally recorded data on grassland management over the years, such as cutting and fertilisation dates and maintenance activities. These observations were integrated when questionnaires were filled out together with the farmers. Values for organic fertilisation with slurry or liquid manure were probably less accurate than those for grazing and mowing, due to the fact that often no exact records existed for the amounts of material put on a particular site. Another source of uncertainty was the variation in $\mathrm{N}$ content of the material, which depended on many factors, for example, the livestock and the amount of added water etc. Here, we gave raw amounts of slurry and liquid manure, as well as the conversion factors to $\mathrm{N}$ per ha (Table 3 ).

The specific management data, presented here, have formed the basis for analyses of land use effects on the biodiversity and ecosystem functioning in grasslands (e.g. Allan et al. 2014, Manning et al. 2015, Klaus et al. 2018)Suppl. material 2. The data can be coupled with climate data and soil information to disentangle effects of management from effects of 
abiotic conditions (Smit et al. 2008). Our data show that ecologists, interested in the effects of land use on biodiversity and ecosystem functions, should pay closer attention to measurements of land use itself, because management can strongly vary between years with significant long-term effects on the target variables measured in any particular year (Klaus et al. 2011, Kleinebecker et al. 2018).

Quality control: Quality assurance took place by checking the plausibility of the values by the grassland experts of the local teams in the following ways i) the answers of the land users were compared with own observations before entering the values in the data table. When uploading the data, the values were again checked ii) by the responsible person for the whole dataset looking at the single values and communicating uncertainties back to the interviewers. Further, unusual values were detected by auxiliary calculations, including minimum and maximum of these values and then double checked with the original hard copy version of the interview.

Step description: The original interviews are stored in hard copies and the information is entered in the joint data table.

The data are stored on the Biodiversity-Exploratories Information System (BExIS) (http:// doi.org/10.17616/R32P9Q) at https://www.bexis.uni-jena.de. This version is in German due to the annual survey of the land users being carried out in German. The interviews of each grassland site is entered in a default excel data sheet and transformed via a visual basic script before it is uploaded to the joint dataset in BExIS.

Daily backups at the BE repository ensures the storage of the actual version of the land use data table. After project end, all datasets are intended to be stored via GFBio in a domain-specific long term archive.

\section{Geographic coverage}

Description: The monitored 150 grassland plots are situated within three regions in Germany, with 50 plots in each region, covering a geographic gradient from the North-East (Schorfheide Chorin), Central Germany (Hainich Dün) and South-West (Schwäbische Alb). The grassland plots experience different land use according to the management.

\section{Taxonomic coverage}

Description: We did not collect any organisms. During the interviews, the land users provided us with information according to their grassland management.

\section{Temporal coverage}

Notes: We obtained land use data of grasslands between 2006 and 2016 . 


\section{Usage rights}

\section{Use license: Other}

IP rights notes: The English version of the dataset is added as supplementary material.

The original, slightly extended, dataset is stored on the Biodiversity-Exploratories Information System (BExIS) (http://doi.org/10.17616/R32P9Q) at https://www.bexis.unijena.de. This version is in German due to the annual survey of the land users being carried out in German. Contact is possible via the Biodiversity Coordination Office (beo@senckenberg.de). Due to sensitive information, such as personal data, the original dataset is not publicly available. Data access can be given by individual request for access. Guidelines can be checked in the data agreement of the BE: https://www.bexis.uni-jena.de/ PublicData/Files/PublicData-DataAgreement.txt.

\section{Data resources}

Data package title: BE_landuse_grassland_2006-2016.csv

\section{Number of data sets: 1}

Data set name: BE_landuse_grassland_2006-2016.csv

Description: The data table (628 KB) contains 1651 rows with records of eleven years on 150 grassland sites, including the variable headers and 116 (Suppl. material 1).

\begin{tabular}{|l|l|}
\hline Column label & Column description \\
\hline Study region & ALB = Schwäbische Alb, HAI = Hainich, SCH = Schorfheide \\
\hline Year & Year of management \\
\hline Date & Date of interview \\
\hline PlotID & $\begin{array}{l}\text { Experimental Plot IDs formatted as }(\mathrm{A}|\mathrm{H}| \mathrm{S}) \text { EG with consecutive numbering. Abbreviations } \\
\text { Grassland, e.g. AEG01 }\end{array}$ \\
\hline Drainage & Measure of drainage and the description of the method (free text) \\
\hline StartDrainage & Starting year of grassland drainage if applicable \\
\hline WaterLogging & Activities on water logging, e.g. for water regulation of fen soils \\
\hline Agriculture1981 & $\begin{array}{l}\text { Use of grassland between } 1981 \text { to } 2006, \text { i.e. (temporal) conversion of grassland into arable } \\
\text { land }\end{array}$ \\
\hline SizeManagementUnit_ha & $\begin{array}{l}\text { Size of the management unit in the survey year, often larger than the } 50 \times 50 \mathrm{~m} \text { study plot } \\
\text { itself }\end{array}$ \\
\hline StartGrazing & Starting month of the first grazing period in the survey year \\
\hline
\end{tabular}




\begin{tabular}{|c|c|}
\hline EndGrazing & End of the last grazing period in the survey year \\
\hline Livestock1 (2-4) & Type of animal in first (second - fourth) grazing period \\
\hline $\begin{array}{l}\text { StartGrazingPeriod1 } \\
(2-4)\end{array}$ & Starting month of first grazing period for livestock $1(2-4)$ \\
\hline EndGrazingPeriod1 (2-4) & Ending month of first grazing period for livestock $1(2-4)$ \\
\hline Cattle6months1 (2-4) & $\begin{array}{l}\text { For Grazing period } 1 \text { (2-4): Number of cattle with an age up to } 6 \text { months (1 cattle up to } 6 \\
\text { month }=0.3 \text { LS }^{*} \text { per day) }\end{array}$ \\
\hline Cattle6-24months1 (2-4) & $\begin{array}{l}\text { For Grazing period } 1(2-4) \text { : Number of cattle with an age between } 6 \text { months and } 2 \text { years } \\
\left(=0.6 \mathrm{LS}^{*}\right)\end{array}$ \\
\hline CattlePlus2years1 (2-4) & For Grazing period $1(2-4)$ : Number of cattle older than 2 years $\left(=1 \mathrm{LS}^{*}\right)$ \\
\hline SheepGoat1year1 (2-4) & For Grazing period $1(2-4)$ : Number of sheep or goats with an age up to 1 year $\left(=0.05 L^{*}\right)$ \\
\hline Pony1 (2-4) & For Grazing period $1(2-4)$ : Number of ponies and small horses $\left(=0.7 L^{*}\right)$ \\
\hline Horse3years1 (2-4) & For Grazing period $1(2-4)$ : Number of horses up to 3 years $\left(=0.7 \mathrm{LS}^{*}\right)$ \\
\hline HorsePlus3years1 (2-4) & For Grazing period $1(2-4)$ : Number of horses older than 3 years $\left(=1.1 \mathrm{LS}^{*}\right)$ \\
\hline NbLivestock1 (2-4) & For Grazing period 1 (2-4): Total number of livestock \\
\hline LivestockUnits1 (2-4) & For Grazing period 1 (2-4): Total sum of the livestock units \\
\hline DayGrazing1 (2-4) & For Grazing period 1 (2-4): duration of grazing (in days) \\
\hline GrazingArea1 (2-4) & For Grazing period 1 (2-4): size of area where livestock grazed \\
\hline Mowing & Number of cuts per year \\
\hline DateMowing1 (2-4) & Date of the first (second-fourth) cut \\
\hline MowingMachine & Type of machine which was used for mowing e.g. rotarymower, doubleknife, mulcher \\
\hline CutWidth_m & Cutting width of the mowing machine \\
\hline CutHeight_cm & Cutting height above soil level of the mowing machine \\
\hline DriveSpeed_kmh & Speed of the mowing machine, normally mean speed value is given \\
\hline MowingConditioner & $\begin{array}{l}\text { Presence of conditioner, i.e. did the mowing machine have a conditioner to improve drying } \\
\text { of the clippings }\end{array}$ \\
\hline Fertilisation & Addition of fertiliser (not including dung depositions by livestock during grazing a parcel) \\
\hline NbFertilisation & Number of fertiliser applications per year \\
\hline DateFertilisation1 (2-7) & Date of first (2nd-7th) fertiliser application \\
\hline Manure_tha & Total amount of applied solid manure \\
\hline Slurry_m3ha & Total amount of applied pig or cow slurry and biogas residues, respectively \\
\hline DescFert & Description of applied organic fertiliser \\
\hline
\end{tabular}




\begin{tabular}{|c|c|}
\hline orgNitrogen_kgNha & Amount of organic nitrogen applied \\
\hline minNitrogen_kgNha & $\begin{array}{l}\text { Amount of nitrogen applied, of mineral origin or the organic fertiliser mash from a bioethanol } \\
\text { factory (see in DescFert) }\end{array}$ \\
\hline totalNitrogen_kgNha & Sum of applied mineral and organic nitrogen [kg N/ha] \\
\hline minPhosphorus_kgPha & $\begin{array}{l}\text { Amount of phosphorus applied [kg } \mathrm{P}_{2} \mathrm{O}_{5} / \text { ha], of mineral origin or mash from a bioethanol } \\
\text { factory (not given for other organic fertilisers) }\end{array}$ \\
\hline minPotassium_kgKha & $\begin{array}{l}\text { Amount of potassium applied }\left[\mathrm{kg} \mathrm{K}_{2} \mathrm{O} / \mathrm{ha}\right] \text {, of mineral origin or mash from a bioethanol } \\
\text { factory (not given for other organic fertilisers) }\end{array}$ \\
\hline Sulphur_kgSha & Total amount of applied Sulphur [kg S/ha] \\
\hline Maintenance & Presence of maintenance measures \\
\hline Levelling & Maintenance to break up matted grass covers \\
\hline DateLevelling & Maintenance: date of levelling \\
\hline Rolling & Maintenance: rolling to level unevenness \\
\hline DateRolling & Maintenance: date of rolling \\
\hline Mulching & $\begin{array}{l}\text { Partial mulching on some spots, e.g. rank patches. The material remains on site after } \\
\text { mowing. We consider this not as a mowing event, as only a small part of the area is treated. }\end{array}$ \\
\hline DateMulching & Date of partial mulching \\
\hline ShrubClearance & $\begin{array}{l}\text { Clearance to avoid shrub encroachment. We consider this not as a mowing event, as only } \\
\text { individual shrubs are targeted. }\end{array}$ \\
\hline DateScrubCl & Date of shrub clearance \\
\hline PlantProtectionAgent & $\begin{array}{l}\text { Pesticide use: pesticides and herbicides. As pesticides in grasslands are very rare and only } \\
\text { used for spot treatment, we do not have further information on this treatment. }\end{array}$ \\
\hline Seeds & Seed addition \\
\hline DescSeeds & Description of usage of the sowing \\
\hline
\end{tabular}

\section{Acknowledgements}

Our special thanks go to all farmers and other stakeholders for providing information on grassland management and their strong support for our project. We thank the manager Martin Gorke and Metke Lilienthal for their work in maintaining the plot and project infrastructure; Simone Pfeiffer, Maren Gleisberg, Christiane Fischer and Jule Mangels for giving support through the central office, Jens Nieschulze, Michael Owonibi and Andreas Ostrowski for managing the central data base and Eduard Linsenmair, Dominik Hessenmöller, Ingo Schöning, François Buscot, Ernst-Detlef Schulze and the late Elisabeth Kalko, for their role in setting up the Biodiversity-Exploratories project.The work has been funded by the DFG Priority Programme 1374 "Infrastructure-Biodiversity- 
Exploratories". Fieldwork permits were issued by the responsible state environmental offices of Baden-Württemberg, Thüringen and Brandenburg).

\section{References}

- Allan E, Bossdorf O, Dormann CF, Prati D, Gossner MM, Tscharntke T, Blüthgen N, Bellach M, Birkhofer K, Boch S, Böhm S, Börschig C, Chatzinotas A, Christ S, Daniel R, Diekötter T, Fischer C, Friedl T, Glaser K, Hallmann C, Hodac L, Hölzel N, Jung K, Klein AM, Klaus VH, Kleinebecker T, Krauss J, Lange M, Morris EK, Müller J, Nacke H, Pasalic E, Rillig MC, Rothenwöhrer C, Schally P, Scherber C, Schulze W, Socher SA, Steckel J, Steffan-Dewenter I, Türke M, Weiner CN, Werner M, Westphal C, Wolters V, Wubet T, Gockel S, Gorke M, Hemp A, Renner SC, Schöning I, Pfeiffer S, König-Ries B, Buscot F, Linsenmair KE, Schulze ED, Weisser WW, Fischer M (2014) Interannual variation in landuse intensity enhances grassland multidiversity. Proceedings of the National Academy of Sciences of the United States of America 111 (1): 308-313. https://doi.org/10.1073/ pnas. 1312213111

- $\quad$ Blüthgen N, Dormann CF, Prati D, Klaus VH, Kleinebecker T, Hölzel N, Alt F, Boch S, Gockel S, Hemp A, Müller J, Nieschulze J, Renner SC, Schöning I, Schumacher U, Socher SA, Wells K, Birkhofer K, Buscot F, Oelmann Y, Rothenwöhrer C, Scherber C, Tscharntke T, Weiner CN, Fischer M, Kalko EKV, Linsenmair KE, Schulze ED, Weisser WW (2012) A quantitative index of land-use intensity in grasslands: Integrating mowing, grazing and fertilization. Basic and Applied Ecology 13 (3): 207-220. https://doi.org/10.1016/ j.baae.2012.04.001

- $\quad$ Boch S, Allan E, Birkhofer K, Bossdorf O, Blüthgen N, Christ-Breulmann S, Diekötter T, Dormann C, Gossner M, Hallmann C, Hemp A, Hölzel N, Jung K, Klaus V, Klein A, Kleinebecker T, Lange M, Müller J, Nacke H, Prati D, Renner S, Rothenwöhrer C, Schall P, Schulze E, Socher S, Tscharntke T, Türke M, Weiner C, Weisser W, Westphal C, Wolters V, Wubet T, Fischer M (2016) Extensive und jährlich wechselnde Nutzungsintensität fördert den Artenreichtum im Grünland - Extensive and inter-annual variation in land-use intensity enhances grassland multidiversity. ANLiegen Natur 38 (1): 86-95.

- $\quad$ Busch V, Klaus VH, Penone C, Schäfer D, Boch S, Prati D, Müller J, Socher SA, Niinemets U, Penuelas J, Hölzel N, Fischer M, Kleinebecker T (2018) Nutrient stoichiometry and land use rather than species richness determine plant functional diversity. Ecology and Evolution 8 (1): 601-616. https://doi.org/10.1002/ece3.3609

- $\quad$ Carlier L, Rotar I, Vlahova M, Vidican R (2009) Importance and functions of grasslands. Notulae Botanicae Horti Agrobotanici Cluj-Napoca 37 (1): 25-30.

- $\quad$ Fischer M, Bossdorf O, Gockel S, Hänsel F, Hemp A, Hessenmöller D, Korte G, Nieschulze J, Pfeiffer S, Prati D, Renner S, Schöning I, Schumacher U, Wells K, Buscot F, Kalko EKV, Linsenmair KE, Schulze ED, Weisser WW (2010) Implementing large-scale and long-term functional biodiversity research: The Biodiversity Exploratories. Basic and Applied Ecology 11 (6): 473-485. https://doi.org/10.1016/j.baae.2010.07.009

- Gossner MM, Lewinsohn TM, Kahl T, Grassein F, Boch S, Prati D, Birkhöfer K, Renner SC, Sikorski J, Wubet T, Arndt H, Baumgärtner V, Blaser S, Blüthgen N, Börschig C, Buscot F, Diekötter T, Jorge LR, Jung K, Keyel AC, Klein AM, Klemmer S, Krauss J, Lange M, Müller J, Overmann J, Pasalic E, Penone C, Perovic DJ, Purschke O, Schall P, Socher SA, 
Sonnemann I, Tschapka M, Tscharntke T, Türke M, Venter PC, Weiner CN, Werner M, Wolters V, Wurst S, Westphal C, Fischer M, Weisser WW, Allan E (2016) Land-use intensification causes multitrophic homogenization of grassland communities. Nature 540 (7632): 266-269. https://doi.org/10.1038/nature20575

- Hönigová I, Vačkáŕ D, Lorencová E, Melichar J, Götzl M, Sonderegger G, Oušková V, Hošek M, Chobot K (2012) Survey on grassland ecosystem services. Report to the EEA European Topic Centre on Biological Diversity. Nature Conservation Agency of the Czech Republic, Prague, 78 pp. [ISBN 978-80-87457-25-2]

- Humbert JY, Ghazoul J, Walter T (2009) Meadow harvesting techniques and their impacts on field fauna. Agriculture Ecosystems \& Environment 130 (1-2): 1-8. https:// doi.org/10.1016/j.agee.2008.11.014

- Humbert JY, Ghazoul J, Richner N, Walter T (2010a) Hay harvesting causes high orthopteran mortality. Agriculture Ecosystems \& Environment 139 (4): 522-527. https:// doi.org/10.1016/j.agee.2010.09.012

- Humbert JY, Ghazoul J, Sauter GJ, Walter T (2010b) Impact of different meadow mowing techniques on field invertebrates. Journal of Applied Entomology 134 (7): 592-599. https:// doi.org/10.1111/j.1439-0418.2009.01503.x

- Klaus VH, Kleinebecker T, Holzel N, Bluthgen N, Boch S, Muller J, Socher SA, Prati D, Fischer M (2011) Nutrient concentrations and fibre contents of plant community biomass reflect species richness patterns along a broad range of land-use intensities among agricultural grasslands. Perspectives in Plant Ecology Evolution and Systematics 13 (4): 287-295. https://doi.org/10.1016/i.ppees.2011.07.001

- Klaus VH, Kleinebecker T, Busch V, Fischer M, Holzel N, Nowak S, Prati D, Schafer D, Schoning I, Schrumpf M, Hamer U (2018) Land use intensity, rather than plant species richness, affects the leaching risk of multiple nutrients from permanent grasslands. Global Change Biology 24 (7): 2828-2840. https://doi.org/10.1111/gcb.14123

- Kleinebecker T, Busch V, Holzel N, Hamer U, Schafer D, Prati D, Fischer M, Hemp A, Lauterbach R, Klaus VH (2018) And the winner is .... ! A test of simple predictors of plant species richness in agricultural grasslands. Ecological Indicators 87: 296-301. https:// doi.org/10.1016/j.ecolind.2017.12.031

- Manning P, Gossner M, Bossdorf O, Allan E, Zhang Y, Prati D, Blüthgen N, Boch S, Böhm S, Börschig C, Hölzel N, Jung K, Klaus V, Klein AM, Kleinebecker T, Krauss J, Lange M, Müller J, Pašalić E, Socher $S$, Tschapka M, Türke M, Weiner C, Werner M, Gockel S, Hemp A, Renner S, Wells K, Buscot F, Kalko EV, Linsenmair KE, Weisser W, Fischer M (2015) Grassland management intensification weakens the associations among the diversities of multiple plant and animal taxa. Ecology 96 (6): 1492-1501. https:// doi.org/10.1890/14-1307.1

- $\quad$ Renner SC, Gossner MM, Kahl T, Kalko EKV, Weisser WW, Fischer M, Allan E (2014) Temporal changes in randomness of bird communities across Central Europe. Plos One 9 (11): e112347. https://doi.org/10.1371/journal.pone.0112347

- Simons NK, Lewinsohn T, Bluthgen N, Buscot F, Boch S, Daniel R, Gossner MM, Jung K, Kaiser K, Muller J, Prati D, Renner SC, Socher SA, Sonnemann I, Weiner CN, Werner M, Wubet T, Wurst S, Weisser WW (2017) Contrasting effects of grassland management modes on species-abundance distributions of multiple groups. Agriculture Ecosystems \& Environment 237: 143-153. https://doi.org/10.1016/j.agee.2016.12.022 
- $\quad$ Smit HJ, Metzger MJ, Ewert F (2008) Spatial distribution of grassland productivity and land use in Europe. Agricultural Systems 98 (3): 208-219. https://doi.org/10.1016/

j.agsy.2008.07.004

- $\quad$ Socher SA, Prati D, Boch S, Muller J, Baumbach H, Gockel S, Hemp A, Schoning I, Wells K, Buscot F, Kalko EKV, Linsenmair KE, Schulze ED, Weisser WW, Fischer M (2013) Interacting effects of fertilization, mowing and grazing on plant species diversity of 1500 grasslands in Germany differ between regions. Basic and Applied Ecology 14 (2): 126-136. https://doi.org/10.1016/i.baae.2012.12.003

- Veen P, Jefferson R, Smidt Jd, der Straaten Jv (2009) Grasslands in Europe of high nature value. KNNV Publishing, the Netherlands [ISBN 978-9-05011-316-8]

\section{Supplementary materials}

\section{Suppl. material 1: BE_landuse_grassland_2006-2016.csv doi}

Authors: Juliane Vogt, Valentin H. Klaus, Ralf Lauterbach, Niclas Otto, Uta Schumacher, Cornelia Fürstenau, Katrin Lorenzen, Andreas Ostrowski, Wolfgang W. Weisser

Data type: utf 8 - txt

Brief description: The present dataset summarises management information collected from 2006 to 2016 for 150 grassland plots in three different regions of Germany. Data are based on annual interviews of the respective farmers, land owners or tenants involved in land management activity, using a standardised questionnaire.

Standardisation of missing values:

"NA" - if not known,

"0" if something was counted but was zero (e.g. no mowing or no cows or no maintenance). Some dates of maintenance or fertilisation are set to "0". For example, in case maintenance measurements were applied on that plot during the year but not the specific one, i.e. mulching was applied and is listed with specific mulching date, but no levelling took place, therefore the levelling date is set to "0" instead of "-1" when generally no maintenance measures were carried out on that plot within the year.

"-1" if not possible, for example, if no mowing a "-1" has been given for the question about mowing machine.

Download file (1.25 MB)

\section{Suppl. material 2: Bibliography of the land use index doi}

Authors: Juliane Vogt

Data type: Text

Brief description: This library shows the citations of the LUI developed by Brüthgen et al. 2012.

Download file $(21.02 \mathrm{~kb})$ 